\title{
Equivalence groupoids and group classification of multidimensional nonlinear Schrödinger equations
}

\author{
Célestin Kurujyibwami ${ }^{\dagger}$ and Roman O. Popovych ${ }^{\ddagger}$ \\ ${ }^{\dagger}$ College of Science and Technology, University of Rwanda, P.O. Box: 3900, Kigali, Rwanda \\ E-mail: celeku@yahoo.fr \\ ¥ Fakultät für Mathematik, Universität Wien, Oskar-Morgenstern-Platz 1, 1090 Wien, Austria \\ Institute of Mathematics of NAS of Ukraine, 3 Tereshchenkivska Str., 01024 Kyiv, Ukraine \\ E-mail: rop@imath.kiev.ua
}

\begin{abstract}
We study admissible and equivalence point transformations between generalized multidimensional nonlinear Schrödinger equations and classify Lie symmetries of such equations. We begin with a wide superclass of Schrödinger-type equations, which includes all the other classes considered in the paper. Showing that this superclass is not normalized, we partition it into two disjoint normalized subclasses, which are not related by point transformations. Further constraining the arbitrary elements of the superclass, we construct a hierarchy of normalized classes of Schrödinger-type equations. This gives us an appropriate normalized superclass for the non-normalized class of multidimensional nonlinear Schrödinger equations with potentials and modular nonlinearities and allows us to partition the latter class into three families of normalized subclasses. After a preliminary study of Lie symmetries of nonlinear Schrödinger equations with potentials and modular nonlinearities for an arbitrary space dimension, we exhaustively solve the group classification problem for such equations in space dimension two.
\end{abstract}

\section{Introduction}

Nonlinear Schrödinger-type equations appear in physics in many contexts. They model nonlinear physical systems in hydrodynamics, optics, acoustics, quantum condensates, heat pulses in solids, plasma physics, quantum mechanics and biomolecular dynamics, to name just a few areas; see for instance $[4,12,23,29,30]$ and references therein.

There is a vast literature on the study of Schrödinger equations within the framework of symmetry analysis of differential equations, including their Lie symmetries and point transformations between them; see, e.g., the reviews in [11], [32, Chapter 17], [56, Section 4] and below. This study was systematically begun in the 1970's with linear Schrödinger equations [9, 39, 40, 41, 42]. Nevertheless, linear Schrödinger equations are quite different from nonlinear ones in symmetry and other related properties, and their study needs specific methods, including modifications of methods of group classification; see [37, 43] and references therein.

To the best of our knowledge, the first paper with essential usage of specific Lie symmetries of nonlinear Schrödinger equations was the paper [64]. It was indicated there that the $(1+2)$-dimensional cubic Schrödinger equations admits additional (conformal) symmetries in comparison with the case of general power modular nonlinearity $|\psi|^{\gamma} \psi$. This displays the fact that the power $\gamma=2$ is critical for space dimension two. Lie symmetries of nonlinear Schrödinger equations were systematically considered for the first time in [10], where an inverse group classification problem for $(1+1)$-dimensional nonlinear Schrödinger equations of the form $i \psi_{t}+\psi_{x x}+F\left(t, x, \psi, \psi^{*}\right)=0$ was solved. More specifically, subalgebras of the essential [57] Lie invariance algebra of the $(1+1)$-dimensional free Schrödinger equations were classified and equations of the above form that are invariant with respect to the listed subalgebras were constructed. Later, other inverse group classification problems for nonlinear Schrödinger equations were also considered, see, e.g., [17, 31, 58, 63]. Some Lie reductions of nonlinear Schrödinger equations with power modular nonlinearity, were carried out in $[1,20,21,28]$. In $[23,24,25,26,27]$, classi- 
cal Lie symmetry analysis was comprehensively carried out for (1+3)-dimensional cubic-quintic Schrödinger equations, whose nonlinear terms are of the form $\left(a_{2}|\psi|^{4}+a_{1}|\psi|^{2}+a_{0}\right) \psi$, where $a_{0}, a_{1}$ and $a_{2}$ are real constants with $\left(a_{1}, a_{2}\right) \neq(0,0)$. This included the computation of the maximal Lie invariance algebras of such equations depending on values of the parameters $a$ 's, the classification of subalgebras of these algebras, Lie reductions using the obtained subalgebras, the construction of exact invariant solutions and additional analysis of some specific submodels like the spherical submodel. Lie reductions and invariant solutions of coupled systems of two $(1+2)-$ dimensional nonlinear Schrödinger equations were considered in [22]. Partially invariant solutions of (1+1)-dimensional nonlinear Schrödinger equations of the form $i \psi_{t}+\psi_{x x}+f \psi+g \psi_{x}=0$ with complex-valued parameter functions $f$ and $g$ of $\left(|\psi|,|\psi|_{x}\right)$ were constructed in [38]. Classical Lie reductions and nonclassical reductions for generalized nonlinear Schrödinger equations of the form $i \psi_{t}+\psi_{x x}+b_{1}\left(|\psi|^{2} \psi\right)_{x}+b_{2}\left(|\psi|^{2}\right)_{x} \psi+a_{2}|\psi|^{4} \psi+a_{1}|\psi|^{2} \psi=0$ with $b_{1}, b_{2} \in \mathbb{C}$, $a_{1}, a_{2} \in \mathbb{R}$ and $\left(b_{1}, b_{2}, a_{2}\right) \neq(0,0,0)$ were carried out in $[11,14,15]$. Analogous reductions were discussed in [11] for the case of space dimension three and in [12] for similar cylindrical equations. The integration of obtained reduced equations gave a number of exact solutions for the above Schrödinger equations. Conditional symmetries of nonlinear Schrödinger equations with modular nonlinearity, $f(|\psi|) \psi$, and of phase Schrödinger equations whose nonlinear terms are of the form $i \beta \psi \arg \psi^{2}+f(|\psi|) \psi$ with $\beta \in \mathbb{R}_{\neq 0}$ were obtained in [18] for specific differential constraints. The systems of determining equations for Lie symmetries of systems of nonlinear Schrödinger equations with inhomogeneous nonlinearities were used in $[66,67,68]$ for testing a software for solving overdetermined systems of PDE's. Special attention was paid to the case of power modular nonlinearity with potentials that are quadratic with respect to space variables. In [44], a new method of group classification, the so-called method of furcate splitting, was suggested, which is especially efficient for group classification of classes of differential equations whose arbitrary elements are functions of one or two arguments. Using this advanced method, the complete group classification of nonlinear Schrödinger equations of the form $i \psi_{t}+\Delta \psi+F\left(\psi, \psi^{*}\right)=0$ was carried out for an arbitrary space dimension. An original method of generalized Bäcklund-Darboux transformations was applied in $[59,60,61,62]$ to finding exact solutions of the coupled matrix nonlinear Schrödinger equations, including those with an external potential. Exact solutions of semilinear radial Schrödinger equations with power modular nonlinearities were constructed in [2] via combining the method of group foliation with separation of variables. Note that a collection of exact solutions of various nonlinear Schrödinger equations was presented in [50].

The algebraic method of group classification was first applied to Schrödinger equations in [29], where admissible transformations and Lie symmetries for variable-coefficient generalizations of $(1+1)$-dimensional cubic Schrödinger equations of the form $i \psi_{t}+G(t, x) \psi_{x x}+W(t, x)|\psi|^{2} \psi+$ $V(t, x) \psi=0$ with complex-valued functions $G, W$ and $V$ of $(t, x)$ with $\operatorname{Re} G \neq 0$ and $\operatorname{Re} W \neq 0$ were studied. Exact solutions of such equations with $G=1$ were considered in [4]. Lie symmetries of more general variable-coefficient cubic-quintic nonlinear Schrödinger equations were classified in [48]. Some results of [58] were extended in [69] via classifying (1+1)-dimensional nonlinear Schrödinger equations of the form $i \psi_{t}+\psi_{x x}+F\left(t, x, \psi, \psi^{*}, \psi_{x}, \psi_{x}^{*}\right)=0$ with Liesymmetry groups of dimensions one, two and three. The equations invariant with respect to the Galilei group and its natural extensions were selected among the listed ones, which was used for symmetry classification of Galilei-invariant complex Doebner-Goldin models; see also [19] for the classical Lie symmetry analysis of these models. Group classification problems for various classes of nonlinear Schrödinger equations with modular nonlinearities and potentials were solved on the series of papers $[33,34,53,54,55]$. Such equations are important for applications and were intensively studied within the framework of group analysis of differential equations, theory of partial differential equations, etc., see e.g. [3, 5, 13, 16, 35, 49]. In fact, the consideration of the above group classification problems stimulated revisiting the entire framework of the algebraic method of group classification, which was based on the notion of normalized class of differential equations $[51,52,56]$. 
In the present paper, we study admissible point transformations within classes of multidimensional generalized nonlinear Schrödinger equations. This allows us to classify Lie symmetries of multidimensional nonlinear Schrödinger equations with potentials and modular nonlinearities using the algebraic method of group classification of differential equations. We essentially generalize results of [56] by considering an arbitrary space dimension and more general classes of nonlinear Schrödinger equations. For this, we combine and further develop various techniques of modern group analysis of differential equations. We apply splitting into normalized subclasses, gauging arbitrary elements by equivalence transformations, looking for normalized superclasses and singling out appropriate subalgebras of the corresponding equivalence algebras. The basic notions and concepts used in this paper, such as class of differential equations, equivalence groupoid, equivalence group, equivalence algebra, group classification and normalization properties of a class of differential equations, can be found in $[6,37,46,51,56,65]$ as well as in references therein. See also $[8,45,47]$ for the general theory of group analysis of differential equations.

Looking for an appropriate normalized superclass for the class of nonlinear Schrödinger equations with potentials and modular nonlinearities, we begin with the study of point transformations within the very wide class $\mathcal{N}$ of generalized $(1+n)$-dimensional $(n \geqslant 2)$ nonlinear Schrödinger equations with "variable mass" of the form

$$
i \psi_{t}+G\left(t, x, \psi, \psi^{*}, \nabla \psi, \nabla \psi^{*}\right) \psi_{a a}+F\left(t, x, \psi, \psi^{*}, \nabla \psi, \nabla \psi^{*}\right)=0,
$$

where $G$ and $F$ are smooth complex-valued functions of their arguments with $G \neq 0$. The class $\mathcal{N}$ is the superclass for all classes of Schrödinger equations considered in the present paper. This class is not normalized but can be partitioned into the two normalized subclasses $\mathcal{N}_{0}$ and $\overline{\mathcal{N}}_{0}$ singled out within the class $\mathcal{N}$ by the constraints $G^{*} \neq-G$ and $G^{*}=-G$, respectively.

Notation. Throughout the paper, $t$ and $x=\left(x_{1}, \ldots, x_{n}\right)$ are the real independent variables, $\psi$ is the unknown complex-valued function of $t$ and $x$, and $\rho:=|\psi|$. For a complex value $\beta$, the notation $\beta^{*}$ denotes its conjugate, and we define $\hat{\beta}=\beta$ if $T_{t}>0$ and $\hat{\beta}=\beta^{*}$ if $T_{t}<0$, where $T$ is $t$-component of point transformations in the space with the coordinates $\left(t, x, \psi, \psi^{*}\right)$. Subscripts of functions denote differentiation with respect to the corresponding variables. The indices $a$ and $b$ run from 1 to $n$, the indices $\mu$ and $\nu$ run from 0 to $n, x_{0}:=t$, and we assume summation over repeated indices. $\nabla \psi=\left(\psi_{1}, \ldots, \psi_{n}\right)$ and $\nabla \psi^{*}=\left(\psi_{1}^{*}, \ldots, \psi_{n}^{*}\right)$. The total derivative operator $\mathrm{D}_{\mu}$ is defined as $\mathrm{D}_{\mu}=\partial_{\mu}+\psi_{\mu} \partial_{\psi}+\psi_{\mu}^{*} \partial_{\psi^{*}}+\psi_{\mu \nu} \partial_{\psi_{\nu}}+\psi_{\mu \nu}^{*} \partial_{\psi_{\nu}^{*}}+\cdots$. Given a class of differential equations, $\pi$ denotes the natural projection of the joint space of the variables and the arbitrary elements on the space of the variables only. $E$ is the $n \times n$ identity matrix.

Choosing specific forms of $G$ and $F$, we further single out several subclasses from the normalized class $\mathcal{N}_{0}$. An important subclass is the normalized class $\overline{\mathcal{F}}$ singled out from $\mathcal{N}_{0}$ by the condition that $G$ is a (nonzero) real constant. The class $\overline{\mathcal{F}}$ is mapped by a family of scalings of $t$ and alternating the sign of this variable, which are equivalence transformations of the class $\overline{\mathcal{F}}$, to its normalized subclass $\mathcal{F}$ associated with the constraint $G=1$, i.e., consisting of equations of the form

$$
i \psi_{t}+\psi_{a a}+F\left(t, x, \psi, \psi^{*}, \nabla \psi, \nabla \psi^{*}\right)=0 .
$$

We combine this consideration of the class $\mathcal{F}$ with $n \geqslant 2$ with earlier results on the class $\mathcal{F}$ with $n=1$ from [56] and thus further assume $n \geqslant 1$. We simultaneously constrain the arbitrary elements $G$ and $F$ by the equations $G=1$ and $F_{\psi_{a}}=F_{\psi_{a}^{*}}=0$ to single out the normalized class $\mathcal{F}_{1}$ whose equations are of the general form

$$
i \psi_{t}+\psi_{a a}+F\left(t, x, \psi, \psi^{*}\right)=0 .
$$

Additionally restricting $F$ to be $F=S(t, x, \rho) \psi$, we obtain the normalized class $\mathcal{S}$ of multidimensional nonlinear Schrödinger equations of the form

$$
i \psi_{t}+\psi_{a a}+S(t, x, \rho) \psi=0, \quad S_{\rho} \neq 0,
$$


where $S$ is an arbitrary smooth complex-valued function of its arguments. After a preliminary study of the Lie symmetries of equations from the class $\mathcal{S}$, we further constrain the arbitrary function $S$ by putting $S(t, x, \rho)=f(\rho)+V(t, x), f_{\rho} \neq 0$. This gives the non-normalized class $\mathcal{V}$ of multidimensional nonlinear Schrödinger equations with potentials and modular nonlinearities of the form

$$
i \psi_{t}+\psi_{a a}+f(\rho) \psi+V(t, x) \psi=0, \quad f_{\rho} \neq 0,
$$

where $V$ is an arbitrary smooth complex-valued potential depending on $t$ and $x$, and $f$ is an arbitrary complex-valued nonlinearity depending only on $\rho$. The class $\mathcal{S}$ is a quite narrow and appropriate normalized superclass of the class $\mathcal{V}$. Using results on the class $\mathcal{S}$, we partition the class $\mathcal{V}$ into normalized subclasses, study properties of Lie symmetries of equations from this class and solve completely the group classification problem for the class $\mathcal{V}$ with $n=2$. We note that the case $n=1$ and the case $f=\rho^{2}$ with $n=2$ were studied in [56].

The paper is organized as follows.

In Section 2 we compute the equivalence groupoid $\mathcal{G}_{\mathcal{N}}$ of the class $\mathcal{N}$. This allows us to find the equivalence group $G \widetilde{\mathcal{N}}$ of the class $\mathcal{N}$ and the equivalence groups $G_{\mathcal{N}_{0}}^{\sim}$ and $G{\widetilde{\tilde{N}_{0}}}$ of its subclasses $\mathcal{N}_{0}$ and $\overline{\mathcal{N}}_{0}$, to check the normalization of these subclasses and to show that equations from these subclasses are not related by point transformations. As a result, we obtain a partition of the equivalence groupoid $\mathcal{G}_{\mathcal{N}}, \mathcal{G}_{\mathcal{N}}^{\sim}=\mathcal{G}_{\mathcal{N}_{0}} \sqcup \mathcal{G}_{\widetilde{\aleph}_{0}}$.

A hierarchy of normalized subclasses of $\mathcal{N}_{0}$ is constructed in Section 3. For each next class in the chain $\mathcal{N}_{0} \supset \tilde{\mathcal{F}} \supset \mathcal{F} \supset \mathcal{F}_{1} \supset \mathcal{S}$, we use results on its directly preceding normalized superclass for describing the equivalence groupoid of this class, which reduces to finding the equivalence group of this class and checking its normalization. For the class $\mathcal{S}$, we additionally derive its equivalence algebra $\mathfrak{g}_{\mathcal{S}}^{\sim}$ as the set constituted by the infinitesimal generators of one-parameter subgroups of its equivalence group $G_{\mathcal{S}}^{\sim}$.

Section 4 is devoted to analyzing properties of the maximal Lie invariance algebras, $\mathfrak{g}_{S}$, of equations, $\mathcal{L}_{S}$, from the class $\mathcal{S}$. Applying the Lie infinitesimal criterion, we derive the system of determining equations for Lie symmetries of equations $\mathcal{L}_{S}$. The analysis of this system yields the kernel invariance algebra $\mathfrak{g}^{\bigcap}$, which is the intersection of all $\mathfrak{g}_{S}$, and a preliminary description of the algebras $\mathfrak{g}_{S}$. This description includes the general form of elements of $\mathfrak{g}_{S}$, the classifying equations for functions and constants parameterizing these elements, the least upper bound of the dimensions of the algebras $\mathfrak{g}_{S}$, which is equal to $n(n+3) / 2+4$, as well as estimates of dimensions of distinguished subalgebras of $\mathfrak{g}_{S}$.

Section 5 deals with the preliminary scheme of group classification for the class $\mathcal{V}$ in the case of an arbitrary space dimension $n$, which is based on the previous study of the class $\mathcal{S}$. We find the equivalence group of the class $\mathcal{V}$ and show that this class, unlike the class $\mathcal{S}$, is not normalized. Then the class $\mathcal{V}$ is partitioned into the disjoint normalized subclasses $\mathcal{V}^{\prime}, \mathcal{P}_{0}$ and $\mathcal{P}_{\lambda}, \lambda \in \mathbb{R}_{\neq 0}$, which are respectively related, up to gauge equivalence transformations in $\mathcal{V}$, to general, logarithmic and power values of the arbitrary element $f$, i.e., $f=f(\rho), f=\delta \ln \rho$ and $f=\delta \rho^{\lambda}$, where $\delta$ is an arbitrary complex constant. The equivalence groups of these subclasses are constructed as well. In order to study Lie symmetries of equations from these subclasses, we treat the nonlinear terms in a special way: by fixing $f(\rho)$ in $\mathcal{V}^{\prime}$ and $\delta$ (after gauging to $|\delta|=1$ and $\delta_{2} \geqslant 0$ by scaling equivalence transformations) in $\mathcal{P}_{\lambda}, \lambda \in \mathbb{R}$, and then considering $V$ as the only arbitrary element. We refine estimates for the dimensions of maximal Lie invariance algebras and of their distinguished subalgebras, which we obtain for equations from the superclass $\mathcal{S}$, for each of the above normalized subclasses with specific nonlinearity $f$.

The complete group classifications of these subclasses in the $(1+2)$-dimensional case are presented in Sections 6.1-6.3, respectively. We introduce $\mathcal{G}_{\mathcal{V}}$-invariant parameters, which depend on values of the arbitrary elements $f$ and $V$ and are related to the dimensions of distinguished subalgebras of the corresponding maximal Lie invariance algebras. The ranges of these parameters can be estimated using the derived estimations of the subalgebra dimensions; in fact, they 
take only small integer values. We use them to distinguish subalgebras of the projections of the equivalence algebras of the above normalized subclasses to the space with the coordinates $\left(t, x, \psi, \psi^{*}\right)$ that are appropriate as the maximal Lie invariance algebras of some equations from these classes. Therefore, it is natural to split the solution of the group classification problems in these subclasses into different cases depending on values of the introduced parameters. In this way, we obtain a complete list of $\mathcal{G}_{\mathcal{v}}$-inequivalent Lie symmetry extensions in the class $\mathcal{V}$ with $n=2$ as the union of the group classification lists for the subclasses $\mathcal{V}^{\prime}, \mathcal{P}_{0}$ and $\mathcal{P}_{\lambda}, \lambda \in \mathbb{R}_{\neq 0}$, up to $G_{\mathcal{V}^{\prime}}{ }^{-}, G_{\mathcal{P}_{0}}{ }^{-}$and $G_{\mathcal{P}_{\lambda}}$-equivalences, respectively.

As mentioned above, the results of the present paper generalize those given in [56] for the $(1+1)$-dimensional nonlinear Schrödinger equations with potentials and modular nonlinearities and for (1+2)-dimensional cubic Schrödinger equations with potentials. It is relevant to also complete the study for the case of spatial dimension three as the most important from the physical point of view. Note that the complexity of group classification of nonlinear Schrödinger equations with potentials and modular nonlinearities exponentially grows when the spatial dimension increases.

We intend to exploit the results of this paper to obtain Lie reductions and invariant solutions for the class of nonlinear Schrödinger equations of the form (5) in future publications.

\section{Admissible and equivalence transformations within the superclass}

Using the direct method, we compute the equivalence groupoid $\mathcal{G} \tilde{\mathcal{N}}$ for the class $\mathcal{N}$ with $n \geqslant 2$, which consists of the equations of the form (1). In the course of this and other computations, we should assume $\left(\psi, \psi^{*}\right)$ to be the tuple of the dependent variables and extend the corresponding arbitrary-element tuple with the complex conjugates of its components, e.g., $(G, F)$ with $G^{*}$ and $F^{*}$. Confining a given differential function of $\left(\psi, \psi^{*}\right)$ to the solution set of an equation from the class $\mathcal{N}$, we should supplement this equation by its complex conjugate, thus considering the system of two equations instead of the single equation; see [37, Section 1]. We find all point transformations of the general form

$$
\varphi: \tilde{t}=T\left(t, x, \psi, \psi^{*}\right), \quad \tilde{x}_{a}=X^{a}\left(t, x, \psi, \psi^{*}\right), \quad \tilde{\psi}=\Psi\left(t, x, \psi, \psi^{*}\right), \quad \tilde{\psi}^{*}=\Psi^{*}\left(t, x, \psi, \psi^{*}\right)
$$

with $\mathrm{d} T \wedge \mathrm{d} X^{1} \wedge \cdots \wedge \mathrm{d} X^{n} \wedge \mathrm{d} \Psi \wedge \mathrm{d} \Psi^{*} \neq 0$, that map a fixed equation $\mathcal{L}_{G F}$ from the class $\mathcal{N}$ to an equation $\mathcal{L}_{\tilde{G} \tilde{F}}$,

$$
i \tilde{\psi}_{\tilde{t}}+\tilde{G}\left(\tilde{t}, \tilde{x}, \tilde{\psi}, \tilde{\psi}^{*}, \tilde{\nabla} \tilde{\psi}, \tilde{\nabla} \tilde{\psi}^{*}\right) \tilde{\psi}_{\tilde{x}_{a} \tilde{x}_{a}}+\tilde{F}\left(\tilde{t}, \tilde{x}, \tilde{\psi}, \tilde{\psi}^{*}, \tilde{\nabla} \tilde{\psi}, \tilde{\nabla} \tilde{\psi}^{*}\right)=0,
$$

from the same class.

Theorem 1. The equivalence groupoid $\mathcal{G}_{\mathcal{N}}$ of the class $\mathcal{N}$ with $n \geqslant 2$ is constituted by triples of the form $((G, F), \varphi,(\tilde{G}, \tilde{F}))$. Here $\varphi$ is a point transformation of the form (6) whose components satisfy the equations

$$
T_{\psi}=T_{\psi^{*}}=0, \quad T_{a}=0, \quad X_{\psi}^{a}=X_{\psi^{*}}^{a}=0, \quad X_{a}^{b} X_{a}^{c}=H \delta_{b c},
$$

for some positive smooth real-valued function $H$ of $(t, x), T_{t} \neq 0, \delta_{b c}$ is the Kronecker delta and,

$$
\text { if } G^{*} \neq-G \text {, then } \Psi_{\psi} \Psi_{\psi^{*}}=0 \text {. }
$$

The transformed arbitrary elements $\tilde{G}$ and $\tilde{F}$ are given by

$$
\tilde{G}=\frac{H}{T_{t}}\left\{\begin{array}{lll}
G & \text { if } \quad \Psi_{\psi} \neq 0, \\
\left(-G^{*}\right) & \text { if } \quad \Psi_{\psi^{*}} \neq 0,
\end{array}\right.
$$




$$
\tilde{F}=\frac{\Psi_{\psi}}{T_{t}} F-\frac{\Psi_{\psi^{*}}}{T_{t}} F^{*}+\left(\hat{\Delta} \Psi+\frac{n-2}{2} \frac{H_{a}}{H} \mathrm{D}_{a} \Psi\right) \frac{\tilde{G}}{H}-\frac{i}{T_{t}}\left(\Psi_{t}-X_{t}^{b} \frac{X_{a}^{b}}{H} \mathrm{D}_{a} \Psi\right),
$$

where $\hat{\Delta}:=\partial_{a a}+2 \psi_{a} \partial_{a \psi}+2 \psi_{a}^{*} \partial_{a \psi^{*}}+\psi_{a} \psi_{a} \partial_{\psi \psi}+2 \psi_{a} \psi_{a}^{*} \partial_{\psi \psi^{*}}+\psi_{a}^{*} \psi_{a}^{*} \partial_{\psi^{*} \psi^{*}}$.

Proof. Let $\varphi$ be a point transformation of the form (6) connecting two equations $\mathcal{L}_{G F}$ and $\mathcal{L}_{\tilde{G} \tilde{F}}$ from the class $\mathcal{N}$. Applying the total derivative operators $\mathrm{D}_{\mu}$ 's to $\tilde{\psi}(\tilde{t}, \tilde{x})=\Psi\left(t, x, \psi, \psi^{*}\right)$ and $\tilde{\psi}^{*}(\tilde{t}, \tilde{x})=\Psi^{*}\left(t, x, \psi, \psi^{*}\right)$, we obtain the equations

$$
\tilde{\psi}_{\tilde{x}_{\nu}} \mathrm{D}_{\mu} X^{\nu}=\mathrm{D}_{\mu} \Psi, \quad \tilde{\psi}_{\tilde{x}_{\nu}}^{*} \mathrm{D}_{\mu} X^{\nu}=\mathrm{D}_{\mu} \Psi^{*}
$$

where we denote $X^{0}:=T$, and $x_{0}:=t$. After rearranging their terms, these equations take the form

$$
\begin{aligned}
& \left(\Psi_{\psi}-\tilde{\psi}_{\tilde{x}_{\nu}} X_{\psi}^{\nu}\right) \psi_{\mu}+\left(\Psi_{\psi^{*}}-\tilde{\psi}_{\tilde{x}_{\nu}} X_{\psi^{*}}^{\nu}\right) \psi_{\mu}^{*}=-\left(\Psi_{\mu}-\tilde{\psi}_{\tilde{x}_{\nu}} X_{\mu}^{\nu}\right), \\
& \left(\Psi_{\psi}^{*}-\tilde{\psi}_{\tilde{x}_{\nu}}^{*} X_{\psi}^{\nu}\right) \psi_{\mu}+\left(\Psi_{\psi^{*}}^{*}-\tilde{\psi}_{\tilde{x}_{\nu}}^{*} X_{\psi^{*}}^{\nu}\right) \psi_{\mu}^{*}=-\left(\Psi_{\mu}^{*}-\tilde{\psi}_{\tilde{x}_{\nu}}^{*} X_{\mu}^{\nu}\right) .
\end{aligned}
$$

Denoting $W=W\left(t, x, \psi, \psi^{*}, \tilde{\nabla} \tilde{\psi}\right):=\Psi-\tilde{\psi}_{\tilde{x}_{\nu}} X^{\nu}$, we solve the above equations with respect to $\psi_{\mu}$ and $\psi_{\mu}^{*}$ :

$$
\psi_{\mu}=-\frac{W_{\mu} W_{\psi^{*}}^{*}-W_{\mu}^{*} W_{\psi^{*}}}{Y}, \quad \psi_{\mu}^{*}=-\frac{W_{\psi} W_{\mu}^{*}-W_{\psi}^{*} W_{\mu}}{Y},
$$

where

$$
\begin{aligned}
Y & :=W_{\psi} W_{\psi^{*}}^{*}-W_{\psi}^{*} W_{\psi^{*}} \\
& =\left|\begin{array}{cc}
\Psi_{\psi} & \Psi_{\psi^{*}} \\
\Psi_{\psi}^{*} & \Psi_{\psi^{*}}^{*}
\end{array}\right|-\left|\begin{array}{cc}
X_{\psi}^{\nu} & X_{\psi^{*}}^{\nu} \\
\Psi_{\psi}^{*} & \Psi_{\psi^{*}}^{*}
\end{array}\right| \tilde{\psi}_{\tilde{x}_{\nu}}-\left|\begin{array}{cc}
\Psi_{\psi} & \Psi_{\psi^{*}} \\
X_{\psi}^{\mu} & X_{\psi^{*}}^{\mu}
\end{array}\right| \tilde{\psi}_{\tilde{x}_{\mu}}^{*}+\left|\begin{array}{cc}
X_{\psi}^{\nu} & X_{\psi^{*}}^{\nu} \\
X_{\psi}^{\mu} & X_{\psi^{*}}^{\mu}
\end{array}\right| \tilde{\psi}_{\tilde{x}_{\nu}} \tilde{\psi}_{\tilde{x}_{\mu}}^{*} .
\end{aligned}
$$

Note that $Y \neq 0$. Indeed, if $Y=0$, then each of the determinants in (9) vanishes, which implies that the tuples $\left(T_{\psi}, X_{\psi}^{1}, \ldots, X_{\psi}^{n}, \Psi_{\psi}, \Psi_{\psi}^{*}\right)$ and $\left(T_{\psi^{*}}, X_{\psi^{*}}^{1}, \ldots, X_{\psi^{*}}^{n}, \Psi_{\psi^{*}}, \Psi_{\psi^{*}}^{*}\right)$ are linearly dependent, leading to a contradiction of the invertibility of the point transformation.

We compute $\psi_{a a}$ using the representations (8) for $\psi_{a}$ :

$$
\begin{aligned}
\psi_{a a} & =\mathrm{D}_{a} \psi_{a}=\psi_{a} \tilde{\psi}_{\tilde{x}_{\mu}} \mathrm{D}_{a} \tilde{\psi}_{\tilde{x}_{\mu}}+\psi_{a} \tilde{\psi}_{\tilde{x}_{\mu}}^{*} \mathrm{D}_{a} \tilde{\psi}_{\tilde{x}_{\mu}}^{*}+R \\
& =\frac{1}{Y}\left(\mathrm{D}_{a} X^{\mu}\right)\left(\mathrm{D}_{a} X^{\nu}\right)\left(\tilde{\psi}_{\tilde{x}_{\mu} \tilde{x}_{\nu}} W_{\psi^{*}}^{*}-\tilde{\psi}_{\tilde{x}_{\mu} \tilde{x}_{\nu}}^{*} W_{\psi^{*}}\right)+R,
\end{aligned}
$$

where $R$ is an expression not involving second derivatives of $\psi$ and $\psi^{*}$, the precise form of which is not essential. We substitute the derived expression for $\psi_{a a}$ into the equation $\mathcal{L}_{G F}$ and then substitute for $\tilde{\psi}_{\tilde{x}_{1} \tilde{x}_{1}}$ and $\tilde{\psi}_{\tilde{x}_{1} \tilde{x}_{1}}^{*}$ in view of the equation $\mathcal{L}_{\tilde{G} \tilde{F}}$ and its conjugate. The obtained equation $\hat{\mathcal{L}}$ can be split with respect to $\tilde{\psi}_{\tilde{t} \tilde{t}}$ and $\tilde{\psi}_{\tilde{t} \tilde{t}}^{*}$. Collecting the coefficients of the first degrees of these derivatives leads to the equations $W_{\psi^{*}}^{*}\left(\mathrm{D}_{a} T\right)\left(\mathrm{D}_{a} T\right)=0$ and $W_{\psi^{*}}\left(\mathrm{D}_{a} T\right)\left(\mathrm{D}_{a} T\right)=0$. Since $\left(W_{\psi^{*}}^{*}, W_{\psi^{*}}\right) \neq(0,0)$ in view of $Y \neq 0$, these equations obviously imply $\left(\mathrm{D}_{a} T\right)\left(\mathrm{D}_{a} T\right)=0$. The last equation can be split with respect to $\psi_{a}$ and $\psi_{a}^{*}$, which leads to $T_{\psi}=T_{\psi^{*}}=0, T_{a} T_{a}=0$. Hence $T_{a}=0$ and $T_{t} \neq 0$. Since $n \geqslant 2$, we can also split the equation $\hat{\mathcal{L}}$ with respect to $\tilde{\psi}_{\tilde{x}_{b}} \tilde{x}_{c}$ and $\tilde{\psi}_{\tilde{x}_{b}}^{*} \tilde{x}_{c}$ with $(b, c) \neq(1,1)$. We collect the coefficients of the first degrees of these derivatives. Taking into account the inequality $\left(W_{\psi^{*}}^{*}, W_{\psi^{*}}\right) \neq(0,0)$, we derive the equations $\left(\mathrm{D}_{a} X^{b}\right)\left(\mathrm{D}_{a} X^{c}\right)=0$, $b \neq c$, and $\left(\mathrm{D}_{a} X^{1}\right)\left(\mathrm{D}_{a} X^{1}\right)=\cdots=\left(\mathrm{D}_{a} X^{n}\right)\left(\mathrm{D}_{a} X^{n}\right)$, which can further be split with respect to $\psi_{a}$ and $\psi_{a}^{*}$. The resulting system includes the equations

$$
\begin{aligned}
& X_{\psi}^{b} X_{\psi}^{c}=0, \quad X_{\psi^{*}}^{b} X_{\psi^{*}}^{c}=0, \quad b \neq c, \quad\left(X_{\psi}^{1}\right)^{2}=\cdots=\left(X_{\psi}^{n}\right)^{2}, \quad\left(X_{\psi^{*}}^{1}\right)^{2}=\cdots=\left(X_{\psi^{*}}^{n}\right)^{2}, \\
& X_{a}^{b} X_{a}^{c}=0, \quad b \neq c, \quad X_{a}^{1} X_{a}^{1}=\cdots=X_{a}^{n} X_{a}^{n}
\end{aligned}
$$


implying $X_{\psi}^{a}=X_{\psi^{*}}^{a}=0$ and $X_{a}^{b} X_{a}^{c}=H \delta_{b c}$ for some positive smooth real-valued function $H$ of $(t, x)$. Using these results for $T$ and $X^{a}$ in (8) we obtain $Y=\Psi_{\psi} \Psi_{\psi^{*}}^{*}-\Psi_{\psi}^{*} \Psi_{\psi^{*}}$,

$$
\begin{aligned}
& \psi_{\mu}=\frac{X_{\mu}^{\nu} \Psi_{\psi^{*}}^{*}}{Y} \tilde{\psi}_{\tilde{x}_{\nu}}-\frac{X_{\mu}^{\nu} \Psi_{\psi^{*}}}{Y} \tilde{\psi}_{\tilde{x}_{\nu}}^{*}-\frac{\Psi_{\mu} \Psi_{\psi^{*}}^{*}-\Psi_{\mu}^{*} \Psi_{\psi^{*}}}{Y}, \\
& \psi_{a a}=\frac{H}{Y}\left(\Psi_{\psi^{*}}^{*} \tilde{\psi}_{\tilde{x}_{b} \tilde{x}_{b}}-\Psi_{\psi^{*}} \tilde{\psi}_{\tilde{x}_{b} \tilde{x}_{b}}^{*}\right)+\tilde{\psi}_{\tilde{x}_{b}} \mathrm{D}_{a} \frac{X_{a}^{b} \Psi_{\psi^{*}}^{*}}{Y}-\tilde{\psi}_{\tilde{x}_{b}}^{*} \mathrm{D}_{a} \frac{X_{a}^{b} \Psi_{\psi^{*}}}{Y}-\mathrm{D}_{a} \frac{\Psi_{a} \Psi_{\psi^{*}}^{*}-\Psi_{a}^{*} \Psi_{\psi^{*}}}{Y} .
\end{aligned}
$$

Due to the above expressions for $\psi_{t}$ and $\psi_{a a}$ we can easily split the equation $\hat{\mathcal{L}}$ with respect to $\tilde{\psi}_{\tilde{t}}$ and $\tilde{\psi}_{\tilde{t}}^{*}$. Collecting the coefficients of the first degrees of these derivatives gives

$$
\left(H G-T_{t} \tilde{G}\right) \Psi_{\psi^{*}}^{*}=0, \quad\left(H G+T_{t} \tilde{G}^{*}\right) \Psi_{\psi^{*}}=0,
$$

and thus the condition (7b) holds since $\left(\Psi_{\psi^{*}}^{*}, \Psi_{\psi^{*}}\right) \neq(0,0)$. If $\Psi_{\psi^{*}}^{*} \Psi_{\psi^{*}} \neq 0$, then $H G=T_{t} \tilde{G}=$ $-T_{t} \tilde{G}^{*}$ and $H G=T_{t} \tilde{G}=-H G^{*}$, i.e., $\tilde{G}=-\tilde{G}^{*}, G=-G^{*}$. Finally, collecting the remaining terms gives the relation between $F$ and $\tilde{F}$.

Remark 2. The determining equations (7a) for elements of the equivalence groupoid $\mathcal{G}_{\mathcal{N}}$ represent the principal structure properties of equations from the class $\mathcal{N}$ with $n \geqslant 2$ or, more precisely, of systems that each consists of such an equation and its complex conjugate. Thus, the equations $T_{\psi}=T_{\psi^{*}}=T_{a}=0, X_{\psi}^{a}=X_{\psi^{*}}^{a}=0$ and $X_{a}^{b} X_{a}^{c}=H \delta_{b c}$ are associated with the evolution form of these systems, their quasi-linearity and the involvement of the derivatives of $\psi$ and $\psi^{*}$ of the highest (second) order via Laplacian, respectively; cf. $[7,36]$ for the case of single equations.

Corollary 3. The class $\mathcal{N}$ is not normalized. Its equivalence group $G \tilde{\mathcal{N}}$ consists of the point transformations in the space of $\left(t, x, \psi, \psi^{*}, \nabla \psi, \nabla \psi^{*}, G, G^{*}, F, F^{*}\right),{ }^{1}$ where the components for $t, x$ and $\psi$ are of the form (6) with $T, X^{a}$ and $\Psi$ satisfying the equations (7a) and $\Psi_{\psi} \Psi_{\psi^{*}}=0$, and the components for $G$ and $F$ are of the form (7b) and (7c), respectively.

Since the class $\mathcal{N}$ is not normalized, we partition it into two disjoint subclasses $\mathcal{N}_{0}$ and $\overline{\mathcal{N}}_{0}$, which are singled out by the constraints $G^{*} \neq-G$ and $G^{*}=-G$, respectively. It is clear that there are no point transformations mapping equations from the class $\mathcal{N}_{0}$ to equations from the class $\overline{\mathcal{N}}_{0}$. We can obtain their corresponding equivalence groupoids $\mathcal{G}_{\mathcal{N}_{0}}$ and $\mathcal{G}_{\widetilde{\aleph}_{0}}$, and as well as their equivalence groups $G_{\widetilde{N}_{0}}^{\sim}$ and $G_{\widetilde{\mathcal{N}}_{0}}^{\sim}$ from Theorem 1 .

Corollary 4. The class $\mathcal{N}$ is partitioned by the constraints $G^{*} \neq-G$ and $G^{*}=-G$ into the two disjoint subclasses $\mathcal{N}_{0}$ and $\overline{\mathcal{N}}_{0}$, respectively, which are normalized and are not related by point transformations. The equivalence group $G_{\tilde{\mathcal{N}}_{0}}$ coincides with the equivalence group $G_{\mathcal{N}}$ of the entire class $\mathcal{N}$. The equivalence group $G_{\widetilde{\mathcal{N}}_{0}}$ consists of the point transformations in the space of $\left(t, x, \psi, \psi^{*}, \nabla \psi, \nabla \psi^{*}, G, G^{*}, F, F^{*}\right)$, where the components for $t, x$ and $\psi$ are of the form (6) with $T, X^{a}$ and $\Psi$ satisfying the equations (7a), and the components for $G$ and $F$ are of the form (7b) and (7c).

\footnotetext{
${ }^{1}$ According to the definition of equivalence groups, the group $G \widetilde{\mathcal{N}}$ acts in the space with the coordinates $\left(t, x, \psi_{(2)}, \psi_{(2)}^{*}, G, G^{*}, F, F^{*}\right)$, where the subscript "(2)" of a dependent variable denotes the collection of jet coordinates corresponding to the derivatives of this dependent variable up to order two, including the dependent variable itself as its zeroth-order derivative. At the same time, the arbitrary elements $F$ and $G$ depend only on the jet variables $\left(t, x, \psi, \psi^{*}, \nabla \psi, \nabla \psi^{*}\right)$. In view of Theorem 1 , admissible transformations in the class $\mathcal{N}$ preserve the subspace of the second-order jet space $\mathrm{J}^{2}\left(t, x \mid \psi, \psi^{*}\right)$ with these coordinates. Hence, we can restrict the space underlying the group $G \tilde{\mathcal{N}}$ and thus assume that its elements act in the space with the coordinates $\left(t, x, \psi, \psi^{*}, \nabla \psi, \nabla \psi^{*}\right)$. It suffices to present only the transformation components for $(t, x, \psi)$ and for the arbitrary elements. The transformation components for derivatives of $\psi$ are constructed from the $(t, x, \psi)$-components by the standard prolongation using the chain rule. The transformation components for derivatives of $\psi^{*}$ and for the complex conjugates of complex-valued arbitrary elements are obtained by conjugating their counterparts for derivatives of $\psi$ and for the corresponding arbitrary elements, cf. [37, Section 1]. We use the same approach for all the subclasses of $\mathcal{N}$ and their reparameterizations considered in the present paper.
} 
In other words, the partition $\mathcal{N}=\mathcal{N}_{0} \sqcup \overline{\mathcal{N}}_{0}$ of the class $\mathcal{N}$ leads to a partition of its equivalence

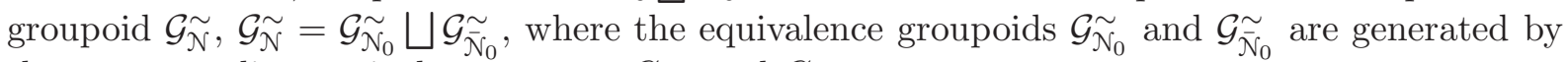
the corresponding equivalence groups $G{\widetilde{\mathcal{N}_{0}}}$ and $G{\widetilde{\mathcal{N}_{0}}}$.

Corollary 5. If a subclass of the class $\mathcal{N}$ intersects both the subclasses $\mathcal{N}_{0}$ and $\overline{\mathcal{N}}_{0}$, then its equivalence group is a subgroup of $G_{\mathcal{N}}$.

\section{Transformational properties of intermediate subclasses}

Selecting the equations with $G=1$ from the class $\mathcal{N}$, we obtain the subclass $\mathcal{F}$ of $\mathcal{N}$ whose equations are of the form (2). It is easy to single out its equivalence groupoid $\mathcal{G}_{\mathcal{F}}$ as a subgroupoid from the equivalence groupoid $\mathcal{G}_{\mathcal{N}}$ computed in Theorem 1 for $n \geqslant 2$. In the case $n=1$, the equivalence groupoid $\mathcal{G}_{\mathcal{F}}$ was computed in [56, Theorem 1]. Merging the above cases of $n$, we formulate the following assertion.

Proposition 6. The equivalence groupoid $\mathcal{G}_{\mathcal{F}}$ of the class $\mathcal{F}$ consists of triples of the form $(F, \varphi, \tilde{F})$, where $\varphi$ is a point transformation in the space of variables, given by

$$
\tilde{t}=T, \quad \tilde{x}_{a}=\left|T_{t}\right|^{1 / 2} O^{a b} x_{b}+\mathcal{X}^{a}, \quad \tilde{\psi}=\Psi(t, x, \hat{\psi}),
$$

and the target value $\tilde{F}$ of the arbitrary element is expressed via its source value $F$ as

$$
\begin{aligned}
\tilde{F}= & \frac{\Psi_{\hat{\psi}}}{\left|T_{t}\right|} \hat{F}-i \frac{\Psi_{t}}{T_{t}}+i\left(\frac{T_{t t}}{2\left|T_{t}\right|^{2}} x_{a}+\frac{\varepsilon^{\prime}}{\left|T_{t}\right|^{3 / 2}} \mathcal{X}_{t}^{b} O^{b a}\right)\left(\Psi_{a}+\Psi_{\hat{\psi}} \hat{\psi}_{a}\right) \\
& -\frac{1}{\left|T_{t}\right|}\left(\Psi_{a a}+2 \Psi_{a \hat{\psi}} \hat{\psi}_{a}+\Psi_{\hat{\psi} \hat{\psi}} \hat{\psi}_{a} \hat{\psi}_{a}\right),
\end{aligned}
$$

where $T$ and $\mathcal{X}^{a}$ are arbitrary smooth real-valued functions of $t$ with $T_{t} \neq 0, \Psi$ is an arbitrary smooth complex-valued function of $t, x$ and $\hat{\psi}$ with $\Psi_{\hat{\psi}} \neq 0, O=\left(O^{a b}\right)$ is an arbitrary constant orthogonal $n \times n$ matrix, and $\varepsilon^{\prime}=\operatorname{sgn} T_{t}$.

Proof. Since the case $n=1$ was studied in [56, Theorem 1], it suffices to consider the case $n \geqslant 2$. Setting $G=1$ and $\tilde{G}=1$ in the description of admissible transformations of $\mathcal{N}$ that is given in Theorem 1, we obtain that transformational parts $\varphi$ of admissible transformations of the class $\mathcal{F}$ are of the form (6), where the components satisfy the equations (7a) with $H=\left|T_{t}\right|$ and the equation $\Psi_{\psi} \Psi_{\psi^{*}}=0$. This expression for $H$ and the condition $\Psi_{\hat{\psi}} \neq 0$ follow from the equation (7b). As a result, we obtain the representation (10a) for $\varphi$. The expression for the target value $\tilde{F}$ of the arbitrary element is derived from (7c) in view of (10a).

The analysis of structure of $\mathcal{G}_{\mathfrak{F}}$ shows that it suffices to choose the space with the coordinates $\left(t, x, \psi, \psi^{*}, \nabla \psi, \nabla \psi^{*}, F, F^{*}\right)$ as the space underlying the equivalence group $G_{\mathcal{F}}^{\sim}$ of the class $\mathcal{F}$.

Corollary 7. The class $\mathcal{F}$ is normalized. The equivalence group $G_{\mathcal{F}}^{\sim}$ of $\mathcal{F}$ is constituted by the point transformations in the space with the coordinates $\left(t, x, \psi, \psi^{*}, \nabla \psi, \nabla \psi^{*}, F, F^{*}\right)$ whose components for $(t, x, \psi, F)$ are of the form (10a)-(10b), and the other components are obtained via the complex conjugation or the prolongation by the chain rule.

The continuous equivalence transformations of the class $\mathcal{F}$ are singled out from the group $G_{\mathcal{F}}$ by the constraints $T_{t}>0$ and $\operatorname{det} O=1$. Therefore, the class $\mathcal{F}$ possesses only two discrete equivalence transformations that are independent up to combining with each other and with continuous equivalence transformations. These are the space reflection $\tilde{t}=t, \tilde{x}_{a}=-x_{a}, \tilde{x}_{b}=x_{b}$, $b \neq a, \tilde{\psi}=\psi, \tilde{F}=F$ for a fixed $a$ and the Wigner time reflection $\tilde{t}=-t, \tilde{x}=x, \tilde{\psi}=\psi^{*}, \tilde{F}=F^{*}$. The above property of the class $\mathcal{F}$ is inherited by its subclasses $\mathcal{F}_{1}, \mathcal{S}, \mathcal{V}, \mathcal{V}^{\prime}, \mathcal{P}_{\lambda}, \lambda \in \mathbb{R}$, which are considered below. 
Remark 8. From the point of view of physical applications, an important class of generalized Schrödinger equations is the wider subclass $\tilde{\mathcal{F}}$ of $\mathcal{N}_{0}$ that is singled out by the constraint that $G$ is a positive real constant, $G \in \mathbb{R}_{>0}$. The class $\tilde{\mathcal{F}}$ is normalized as well. Similarly to the class $\mathcal{F}$, the equivalence group of $\tilde{\mathcal{F}}$ consists of the point transformations in the space with the coordinates $\left(t, x, \psi, \psi^{*}, \nabla \psi, \nabla \psi^{*}, G, F, F^{*}\right)$ whose $(t, \psi, F)$-components are given by (10a)(10b), the $x$-components is modified, in comparison to (10a), as $\tilde{x}_{a}=c\left|T_{t}\right|^{1 / 2} O^{a b} x_{b}+\mathcal{X}^{a}$, the $G$-component is $\tilde{G}=c^{2} G$ and the other components are again obtained via the complex conjugation or the prolongation by the chain rule. Here $c$ runs through $\mathbb{R}_{>0}$. The extension of $\mathcal{F}$ to $\tilde{\mathcal{F}}$ is not essential since the class $\tilde{\mathcal{F}}$ can be mapped to its subclass $\mathcal{F}$ via gauging of $G$ by scalings of $x$. Relaxing the constraint $G \in \mathbb{R}_{>0}$ to $G \in \mathbb{R}_{\neq 0}$ merely introduce the simultaneous alternating of the signs of $t, F$ and $G$ into the corresponding equivalence group. In the same way, the constraint $G=1$ can be relaxed to $G \in \mathbb{R}_{>0}$ or $G \in \mathbb{R}_{\neq 0}$ for the subclasses of $\mathcal{F}$ considered below, and these relaxations are also not essential in the course of the study of admissible transformations and Lie symmetries.

Constraining the arbitrary element $F$ with the equations $F_{\psi_{a}}=F_{\psi_{a}^{*}}=0$, we single out the subclass $\mathcal{F}_{1}$, whose equations are of the form (3). Then the expression (10b) for $\tilde{F}$ implies the equations $\Psi_{a \hat{\psi}}=\frac{1}{2} X_{b}^{a} X_{t}^{b} \Psi_{\hat{\psi}}$ and $\Psi_{\hat{\psi} \hat{\psi}}=0$. Integrating them, we obtain the expression for $\Psi$ and then substitute it into (10), which gives the description of the admissible transformations of the class $\mathcal{F}_{1}$.

Proposition 9. The class $\mathcal{F}_{1}$ is normalized. Its equivalence group $G_{\mathcal{F}_{1}}$ is a subgroup of $G_{\tilde{F}}$ and consists of point transformations of the form (10a)- (10b), where

$$
\Psi=\exp \left(\frac{i}{8} \frac{T_{t t}}{\left|T_{t}\right|} x_{a} x_{a}+\frac{i}{2} \frac{\varepsilon^{\prime} \mathcal{X}_{t}^{b}}{\left|T_{t}\right|^{1 / 2}} O^{b a} x_{a}+i \Sigma+Z\right) \hat{\psi}+\Psi^{0}
$$

$T, \mathcal{X}^{a}, \Sigma$ and $Z$ are arbitrary smooth real-valued functions of $t$ with $T_{t} \neq 0, \Psi^{0}$ is an arbitrary smooth complex-valued function of $t$ and $x$, and $O=\left(O^{a b}\right)$ is an arbitrary constant orthogonal $n \times n$ matrix.

We now turn our attention to the subclass of $\mathcal{F}_{1}$ singled out by the constraints

$$
\psi\left(\frac{F}{\psi}\right)_{\psi}-\psi^{*}\left(\frac{F}{\psi}\right)_{\psi^{*}}=0, \quad \psi\left(\frac{F}{\psi}\right)_{\psi}+\psi^{*}\left(\frac{F}{\psi}\right)_{\psi^{*}} \neq 0
$$

which are jointly equivalent to the representation $F=S(t, x, \rho) \psi$ with $S_{\rho} \neq 0$, where $\rho:=|\psi|$. The reparameterization of this subclass by assuming $S:=F / \psi$ to be the arbitrary element instead of $F$ leads to the class $\mathcal{S}$ of equations of the form (4). In terms of $S$, the above auxiliary system for the arbitrary element takes the form

$$
\psi S_{\psi}-\psi^{*} S_{\psi^{*}}=0, \quad \psi S_{\psi}+\psi^{*} S_{\psi^{*}} \neq 0
$$

Proposition 9 implies that the equivalence group $G_{\tilde{\delta}}^{\sim}$ of the class $\mathcal{S}$ is imbedded as a subgroup into the group $G_{\mathcal{F}_{1}}$, which is associated with the constraint $\Psi^{0}=0$. A similar claim holds for the equivalence groupoid of the class $\mathcal{S}$.

Theorem 10. The class $\mathcal{S}$ is normalized. Its equivalence group $G_{\mathcal{S}}^{\sim}$ consists of the point transformations in the space with the coordinates $\left(t, x, \psi, \psi^{*}, S, S^{*}\right)$ that are of the form

$$
\begin{aligned}
& \tilde{t}=T, \quad \tilde{x}_{a}=\left|T_{t}\right|^{1 / 2} O^{a b} x_{b}+\mathcal{X}^{a}, \\
& \tilde{\psi}=\exp \left(\frac{i}{8} \frac{T_{t t}}{\left|T_{t}\right|} x_{a} x_{a}+\frac{i}{2} \frac{\varepsilon^{\prime} \mathcal{X}_{t}^{b}}{\left|T_{t}\right|^{1 / 2}} O^{b a} x_{a}+i \Sigma+Z\right) \hat{\psi},
\end{aligned}
$$




$$
\tilde{S}=\frac{\hat{S}}{\left|T_{t}\right|}+\frac{2 T_{t t t} T_{t}-3 T_{t t}^{2}}{16 \varepsilon^{\prime} T_{t}^{3}} x_{a} x_{a}+\frac{\varepsilon^{\prime}}{2}\left(\frac{\mathcal{X}_{t}^{b}}{T_{t}}\right)_{t} \frac{O^{b a} x_{a}}{\left|T_{t}\right|^{1 / 2}}+\frac{\Sigma_{t}-i Z_{t}}{T_{t}}-\frac{\mathcal{X}_{t}^{a} \mathcal{X}_{t}^{a}+i n T_{t t}}{4 T_{t}^{2}} .
$$

Here $T, \mathcal{X}^{a}, Z$ and $\Sigma$ are arbitrary smooth real-valued functions of $t$ with $T_{t} \neq 0, \varepsilon^{\prime}=\operatorname{sgn} T_{t}$ and $O=\left(O^{a b}\right)$ is an arbitrary constant orthogonal $n \times n$ matrix.

Theorem 10 is important for the group classification of any subclass of the class $\mathcal{S}$. Admissible and equivalence transformations for any such subclass are deduced from transformations of the form (12).

Corollary 11. The ratio $\rho S_{\rho \rho} / S_{\rho}$ is a differential invariant of the subgroup of $G_{\S}^{\sim}$ associated with the condition $T_{t}>0$. Moreover, if this ratio is real-valued, then it is a differential invariant of the entire group $G_{\mathcal{S}}^{\sim}$.

To find the equivalence algebra $\mathfrak{g}_{\mathcal{S}}^{\sim}$ of the class $\mathcal{S}$, we use the knowledge of the equivalence group $G_{\mathcal{S}}$ of the class $\mathcal{S}$ as described in Theorem 10. We evaluate the set of all infinitesimal generators of one-parameter subgroups of the group $G_{\mathcal{S}}^{\sim}$ by representing the parameter function $\Sigma$ as $\Sigma=\frac{1}{4} \mathcal{X}^{a} \mathcal{X}_{t}^{a}+\bar{\Sigma}$, where $\bar{\Sigma}$ is a smooth function of $t$, to ensure the existence of such subgroups. Then, we successively assume one of the parameters $T, O, \mathcal{X}^{a}, \bar{\Sigma}$ and $Z$ to depend on a continuous parameter $\delta$ and setting the other parameters to their trivial values, which are $t$ for $T, E$ for $O$ and zeroes for $\mathcal{X}^{a}, \bar{\Sigma}$ and $Z$. This procedure leads to the components of the associated infinitesimal generator $\tau \partial_{t}+\xi^{a} \partial_{a}+\eta \partial_{\psi}+\eta^{*} \partial_{\psi^{*}}+\theta \partial_{S}+\theta^{*} \partial_{S^{*}}$, computed as

$$
\tau=\left.\frac{\mathrm{d} \tilde{t}}{\mathrm{~d} \delta}\right|_{\delta=0}, \quad \xi^{a}=\left.\frac{\mathrm{d} \tilde{x}}{\mathrm{~d} \delta}\right|_{\delta=0}, \quad \eta=\left.\frac{\mathrm{d} \tilde{\psi}}{\mathrm{d} \delta}\right|_{\delta=0}, \quad \theta=\left.\frac{\mathrm{d} \tilde{S}}{\mathrm{~d} \delta}\right|_{\delta=0} .
$$

Corollary 12. The equivalence algebra of the class $\mathcal{S}$ is the algebra

$$
\mathfrak{g}_{\mathfrak{S}}=\left\langle\hat{D}(\tau), \hat{J}_{a b}, a<b, \hat{P}(\chi), \hat{M}(\sigma), \hat{I}(\zeta)\right\rangle,
$$

where $\chi:=\left(\chi^{1}, \ldots, \chi^{n}\right) ; \tau, \chi^{a}, \sigma$ and $\zeta$ run through the set of smooth real-valued functions of $t$,

$$
\begin{aligned}
\hat{D}(\tau)= & \tau \partial_{t}+\frac{1}{2} \tau_{t} x_{a} \partial_{a}+\frac{i}{8} \tau_{t t} x_{a} x_{a}\left(\psi \partial_{\psi}-\psi^{*} \partial_{\psi^{*}}\right) \\
& -\left(\tau_{t} S-\frac{1}{8} \tau_{t t t} x_{a} x_{a}+i \frac{\tau_{t t}}{4}\right) \partial_{S}-\left(\tau_{t} S^{*}-\frac{1}{8} \tau_{t t t} x_{a} x_{a}-i \frac{\tau_{t t}}{4}\right) \partial_{S^{*}}, \\
\hat{J}_{a b}=x_{a} \partial_{b}-x_{b} \partial_{a}, \quad a \neq b & \\
\hat{P}(\chi)= & \chi^{a} \partial_{a}+\frac{i}{2} \chi_{t}^{a} x_{a}\left(\psi \partial_{\psi}-\psi^{*} \partial_{\psi^{*}}\right)+\frac{1}{2} \chi_{t t}^{a} x_{a}\left(\partial_{S}+\partial_{S^{*}}\right), \\
\hat{M}(\sigma)= & i \sigma\left(\psi \partial \psi-\psi \partial \psi^{*}\right)+\sigma_{t}\left(\partial_{S}+\partial_{S}^{*}\right), \\
\hat{I}(\zeta)= & \zeta\left(\psi \partial \psi+\psi^{*} \partial \psi^{*}\right)-i \zeta_{t}\left(\partial_{S}+\partial_{S}^{*}\right) .
\end{aligned}
$$

\section{Preliminary analysis of Lie symmetries of equations from an intermediate class}

For a fixed value of the arbitrary element $S$, let $\mathfrak{g}_{S}$ denote the maximal Lie invariance algebra of the corresponding equation $\mathcal{L}_{S}$ from the class $\mathcal{S}$. Elements of $\mathfrak{g}_{S}$ are vector fields in the space of variables $\left(t, x, \psi, \psi^{*}\right)$ of the form $Q=\tau \partial_{t}+\xi^{a} \partial_{a}+\eta \partial_{\psi}+\eta^{*} \partial_{\psi^{*}}$, where the components $\tau$ and $\xi^{a}$ (resp. $\eta$ ) are real-valued (resp. complex-valued) smooth functions of $\left(t, x, \psi, \psi^{*}\right)$ that satisfy the infinitesimal invariance criterion for the equation $\mathcal{L}_{S}$,

$$
\left.Q_{(2)}\left(i \psi_{t}+\psi_{a a}+S(t, x, \rho) \psi\right)\right|_{\mathcal{L}_{S}}=\left.\left(i \eta^{t}+\eta^{a a}+\left(\tau S_{t}+\xi^{a} S_{a}\right) \psi+\rho S_{\rho} \eta\right)\right|_{\mathcal{L}_{S}}=0
$$


Here $\eta^{*}$ is the complex conjugate of $\eta \cdot Q_{(2)}$ is the second prolongation of the vector field $Q$ whose components $\eta^{t}$ and $\eta^{a b}$ correspond to the jet coordinates $\psi_{t}$ and $\psi_{a b}$, respectively,

$$
\eta^{t}=\mathrm{D}_{t}\left(\eta-\tau \psi_{t}-\xi^{a} \psi_{a}\right)+\tau \psi_{t t}+\xi^{a} \psi_{t a}, \quad \eta^{a b}=\mathrm{D}_{a} \mathrm{D}_{b}\left(\eta-\tau \psi_{t}-\xi^{c} \psi_{c}\right)+\tau \psi_{t a b}+\xi^{c} \psi_{a b c} .
$$

Recall that $\mathrm{D}_{t}$ and $\mathrm{D}_{a}$ are the operators of total derivatives with respect to $t$ and $x_{a}$, respectively. We expand the condition (13), confine it to the manifold defined by the equation $\mathcal{L}_{S}$ in the underlying jet space by substituting $\psi_{t}=i \psi_{a a}+i S \psi$ and $\psi_{t}^{*}=-i \psi_{a a}^{*}-i S^{*} \psi^{*}$ and split the obtained equation with respect to the parametric derivatives $\psi_{t a}, \psi_{t a}^{*}, \psi_{a b}, \psi_{a b}^{*}, \psi_{a}$ and $\psi_{a}^{*}$. After a re-arrangement, we derive the system of determining equations for the components of a vector field $Q \in \mathfrak{g}_{S}$,

$$
\begin{aligned}
& \tau_{\psi}=\tau_{\psi^{*}}=\tau_{a}=0, \quad \xi_{\psi}^{a}=\xi_{\psi^{*}}^{a}=0, \quad \tau_{t}=2 \xi_{1}^{1}=\cdots=2 \xi_{n}^{n}, \quad \xi_{b}^{a}+\xi_{a}^{b}=0, a \neq b, \\
& \eta_{\psi^{*}}=\eta_{\psi \psi}=0, \quad 2 \eta_{\psi a}=i \xi_{t}^{a}, \quad \psi \eta_{\psi}=\eta, \\
& i \eta_{t}+\eta_{a a}+\left(\tau S_{t}+\xi^{a} S_{a}\right) \psi+\rho S_{\rho} \operatorname{Re} \eta_{\psi}+\tau_{t} S=0 .
\end{aligned}
$$

The general solution of the subsystem (14a)-(14b) is

$$
\tau=\tau(t), \quad \xi^{a}=\frac{1}{2} \tau_{t} x_{a}+\kappa_{a b} x_{b}+\chi^{a}, \quad \eta=\left(\frac{i}{8} \tau_{t t} x_{a} x_{a}+\frac{i}{2} \chi^{a} x_{a}+i \sigma+\zeta\right) \psi,
$$

where $\tau, \chi^{a}, \sigma$ and $\zeta$ are smooth real-valued functions of $t$, and $\left(\kappa_{a b}\right)$ is a constant skewsymmetric matrix. Substituting these expressions into the equation (14c), we get the classifying condition for the Lie symmetry vector fields of equations from the class $\mathcal{S}$.

Theorem 13. The maximal Lie invariance algebra $\mathfrak{g}_{S}$ of an equation $\mathcal{L}_{S}$ from the class $\mathcal{S}$ consists of the vector fields of the form $Q=D(\tau)-\sum_{a<b} \kappa_{a b} J_{a b}+P(\chi)+\sigma M+\zeta I$, where

$$
\begin{aligned}
& D(\tau)=\tau \partial_{t}+\frac{1}{2} \tau_{t} x_{a} \partial_{a}+\frac{1}{8} \tau_{t t} x_{a} x_{a} M, \quad J_{a b}=x_{a} \partial_{b}-x_{b} \partial_{a}, \quad a \neq b, \\
& P(\chi)=\chi^{a} \partial_{a}+\frac{1}{2} \chi_{t}^{a} x_{a} M, \quad M=i \psi \partial_{\psi}-i \psi^{*} \partial_{\psi^{*}}, \quad I=\psi \partial_{\psi}+\psi^{*} \partial_{\psi^{*}},
\end{aligned}
$$

the parameters $\tau, \chi^{a}, \sigma$ and $\zeta$ are arbitrary real-valued smooth functions of $t$ and $\left(\kappa_{a b}\right)$ is an arbitrary constant skew-symmetric $n \times n$ matrix that together satisfy the classifying condition

$$
\tau S_{t}+\left(\frac{1}{2} \tau_{t} x_{a}+\kappa_{a b} x_{b}+\chi^{a}\right) S_{a}+\zeta \rho S_{\rho}+\tau_{t} S=\frac{1}{8} \tau_{t t t} x_{a} x_{a}+\frac{1}{2} \chi_{t t}^{a} x_{a}+\sigma_{t}-i \zeta_{t}-i \frac{n}{4} \tau_{t t} .
$$

Varying the arbitrary element $S$ and splitting the classifying condition (15) with respect to derivatives of $S$, we find the system of determining equations for elements of the kernel Lie invariance algebra $\mathfrak{g}^{\cap}$ of equations from the class $\mathcal{S}, \tau=\chi=\sigma_{t}=\zeta=0$ and $\kappa_{a b}=0$, which implies $\mathfrak{g}^{\cap}=\langle M\rangle$. Choosing various appropriate values of $S$ in Theorem 10, we show that the common point transformations of equations from the class $\mathcal{S}$ are of the form $(12 \mathrm{a})-(12 \mathrm{~b})$ with $T=t, O=E$ and $\mathcal{X}^{a}=0=\Sigma_{t}=Z=0$.

Proposition 14. The kernel point-symmetry group $G^{\cap}$ of equations from the class $\mathcal{S}$ is constituted by the point transformations $\tilde{t}=t, \tilde{x}=x, \tilde{\psi}=e^{i c} \psi$, where $c$ is an arbitrary real constant. The Lie algebra of $G^{\cap}$ coincides with $\mathfrak{g}^{\cap}=\langle M\rangle$.

Consider the linear span $\mathfrak{g}_{\langle\rangle}$of all the maximal Lie invariance algebras of equations from the class $\mathcal{S}, \mathfrak{g}_{\langle\rangle}:=\sum_{S} \mathfrak{g}_{S}$. For any vector field $Q$ of the general form from Theorem 13, where at least one of the parameters $\tau, \kappa_{a b}, \chi^{a}$ and $\zeta$ takes a nonzero value, there are values of the arbitrary 
element $S$ that each satisfies, together with the components of $Q$, the classifying condition (15), and thus the corresponding algebra $\mathfrak{g}_{S}$ contains $Q$. Therefore,

$$
\mathfrak{g}_{\langle\rangle}:=\left\langle D(\tau), J_{a b}, P(\chi), \sigma M, \zeta I\right\rangle,
$$

where the parameter functions $\tau, \chi^{a}, \sigma$ and $\zeta$ run through the set of real-valued smooth functions of $t$. The nonzero commutation relations between vector fields spanning $\mathfrak{g}_{\langle>}$are

$$
\begin{aligned}
& {\left[D\left(\tau^{1}\right), D\left(\tau^{2}\right)\right]=D\left(\tau^{1} \tau_{t}^{2}-\tau^{2} \tau_{t}^{1}\right), \quad[D(\tau), P(\chi)]=P\left(\tau \chi_{t}-\frac{\tau_{t}}{2} \chi\right),} \\
& {[D(\tau), \sigma M]=\tau \sigma_{t} M, \quad[D(\tau), \zeta I]=\tau \zeta_{t} I, \quad\left[J_{a b}, J_{b c}\right]=J_{a c}, \quad a \neq b \neq c \neq a,} \\
& {\left[J_{a b}, P(\chi)\right]=P(\hat{\chi}) \quad \text { with } \quad \hat{\chi}^{a}=\chi^{b}, \quad \hat{\chi}^{b}=-\chi^{a}, \quad \chi^{c}=0, \quad a \neq b \neq c \neq a,} \\
& {[P(\chi), P(\tilde{\chi})]=\frac{1}{2}\left(\chi^{a} \tilde{\chi}_{t}^{a}-\tilde{\chi}^{a} \chi_{t}^{a}\right) M .}
\end{aligned}
$$

From these commutation relations, it is clearly seen that the space $\mathfrak{g}_{\langle\rangle}$is closed with respect to the Lie bracket of vector fields and thus is a Lie algebra. The subspaces $\langle\sigma M\rangle,\langle\zeta I\rangle,\langle P(\chi), \sigma M\rangle$, $\langle P(\chi), \sigma M, \zeta I\rangle,\left\langle J_{a b}, P(\chi), \sigma M\right\rangle,\left\langle J_{a b}, P(\chi), \sigma M, \zeta I\right\rangle$ and $\langle D(\tau), P(\chi), \sigma M, \zeta I\rangle$ are ideals of $\mathfrak{g}_{\langle\rangle}$. Furthermore, the subspaces $\langle D(\tau)\rangle,\left\langle J_{a b}\right\rangle$ and $\left\langle D(\tau), J_{a b}\right\rangle$ are subalgebras of $\mathfrak{g}_{\langle\rangle}$.

The algebra $\mathfrak{g}_{\langle\rangle}$coincides, in view of Corollary 12 , with the projection $\pi_{*} \mathfrak{g}_{\mathcal{S}}^{\sim}$ of $\mathfrak{g}_{\mathcal{S}}^{\sim}$ to the space with the coordinates $\left(t, x, \psi, \psi^{*}\right)$. Therefore, within the framework of the algebraic method, the group classification of the class $\mathcal{S}$ reduces to the classification of the appropriate subalgebras of $\mathfrak{g}_{\langle>}$up to $\pi_{*} G_{\mathcal{S}}^{\sim}$-equivalence.

Definition 15. A subalgebra $\mathfrak{s}$ of $\mathfrak{g}_{\langle>}$is said to be appropriate if there exists an arbitrary smooth function $S$ such that $\mathfrak{s}=\mathfrak{g}_{S}$.

By $\mathcal{D}(T), \mathcal{J}(O), \mathcal{P}(\mathcal{X})$ with $\mathcal{X}=\left(\mathcal{X}^{1}, \ldots, \mathcal{X}^{n}\right), \mathcal{M}(\Sigma)$ and $\mathcal{I}(Z)$ we respectively denote the point transformations in the space with the coordinates $\left(t, x, \psi, \psi^{*}\right)$ that are of the form (12a)(12b), where the parameter-functions $T, O, \mathcal{X}^{a}, \Sigma$ and $Z$, successively excluding one of them, are set to the values corresponding to the identity transformation, which are $t$ for $T, E$ for $O$ and zeroes for $\mathcal{X}^{a}, \Sigma$ and $Z$. These elementary transformations generate the entire group $\pi_{*} G^{\sim}$. The nonidentity pushforward actions of elementary transformations from $\pi_{*} G^{\sim}$ on the vector fields spanning $\mathfrak{g}_{\langle>}$are

$$
\begin{aligned}
& \mathcal{D}_{*}(T) D(\tau)=D(\tilde{\tau}), \quad \mathcal{D}_{*}(T) P(\chi)=P(\tilde{\chi}), \quad \mathcal{D}_{*}(T)(\sigma M)=\tilde{\sigma} \tilde{M}, \quad \mathcal{D}_{*}(T)(\zeta I)=\tilde{\zeta} \tilde{I}, \\
& \mathcal{J}_{*}(O) P(\chi)=\tilde{P}(O \chi), \\
& \mathcal{P}_{*}(\mathcal{X}) D(\tau)=\tilde{D}(\tau)+\tilde{P}\left(\tau \mathcal{X}_{t}-\frac{\tau_{t}}{2} \mathcal{X}\right)+\left(\frac{\tau_{t t}}{8} \mathcal{X}^{a} \mathcal{X}^{a}-\frac{\tau_{t}}{4} \mathcal{X}^{a} \mathcal{X}_{t}^{a}-\frac{\tau}{2} \mathcal{X}^{a} \mathcal{X}_{t t}^{a}\right) \tilde{M}, \\
& \mathcal{P}_{*}(\mathcal{X}) J_{a b}=\tilde{J}_{a b}+P(\hat{\mathcal{X}})-\frac{1}{2}\left(\mathcal{X}^{a} \mathcal{X}_{t}^{b}-\mathcal{X}^{b} \mathcal{X}_{t}^{a}\right) \tilde{M}, \\
& \mathcal{P}_{*}(\mathcal{X}) P(\chi)=\tilde{P}(\chi)+\frac{1}{2}\left(\chi^{a} \mathcal{X}_{t}^{a}-\chi_{t}^{a} \mathcal{X}^{a}\right) \tilde{M}, \\
& \mathcal{M}_{*}(\Sigma) D(\tau)=\tilde{D}(\tau)+\tau \Sigma_{t} \tilde{M}, \quad \mathcal{I}_{*}(Z) D(\tau)=\tilde{D}(\tau)+\tau Z_{t} \tilde{I} .
\end{aligned}
$$

Here tildes over the vector fields mean that these vector fields are expressed in the new variables, where $\tilde{\tau}(\tilde{t})=\left(T_{t} \tau\right)\left(T^{-1}(\tilde{t})\right), \tilde{\chi}(\tilde{t})=\left(\left|T_{t}\right|^{1 / 2} \chi\right)\left(T^{-1}(\tilde{t})\right), \hat{\mathcal{X}}^{a}=\mathcal{X}^{b}, \hat{\mathcal{X}}^{b}=-\mathcal{X}^{a}, \hat{\mathcal{X}}^{c}=0, c \neq a, b$, $\tilde{\sigma}=\sigma\left(T^{-1}(\tilde{t})\right), \tilde{\rho}=\rho\left(T^{-1}(\tilde{t})\right)$, and in each pushforward by $\mathcal{D}_{*}(T)$ we should substitute the expression for $t$ given by inverting the relation $\tilde{t}=T(t)$, whereas $t=\tilde{t}$ for the other pushforwards.

Lemma 16. $\mathfrak{g}_{S} \cap\langle\sigma M, \zeta I\rangle=\langle M\rangle=\mathfrak{g}^{\cap}$ for any $S$ with $\left(\rho S_{\rho}\right)_{\rho} \neq 0$ or with $S_{a \rho} \neq 0$ for some a. 
Proof. If $\tau=0, \chi^{a}=0$ and $\kappa_{a b}=0$, then the classifying condition (15) reduces to $\zeta \rho S_{\rho}=\sigma_{t}-i \zeta_{t}$. Differentiating this equation with respect to $\rho$ and $x_{a}$, we derive $\zeta\left(\rho S_{\rho}\right)_{\rho}=\zeta \rho S_{a \rho}=0$, which implies $\zeta=0$. Then $\sigma_{t}=0$.

Lemma 17. $\mathfrak{g}_{S} \cap\langle\sigma M\rangle=\langle M\rangle$ for any $S$ with $S_{\rho} \neq 0$.

Proof. For $\tau=0, \chi^{a}=0, \kappa_{a b}=0$ and $\zeta=0$, the classifying condition (15) reduces to $\sigma_{t}=0$.

Lemma 18. $\operatorname{dim} \mathfrak{g}_{S} \leqslant \frac{n(n+3)}{2}+4$ for any $S$ with $S_{\rho} \neq 0$, and this upper bound is the least.

Proof. The proof is similar to the one of Lemma 1 in [37]. For each fixed value of the arbitrary element $S$, the classifying condition (15) yields a system of linear ordinary differential equations of the following form:

$$
\begin{aligned}
& \tau_{t t t}=\gamma^{00} \tau_{t}+\gamma^{01} \tau+\gamma^{0, a+1} \chi^{a}+\gamma^{0, n+2} \zeta+\theta^{0 a b} \kappa_{a b}, \\
& \chi_{t t}^{c}=\gamma^{c 0} \tau_{t}+\gamma^{c 1} \tau+\gamma^{c, a+1} \chi^{a}+\gamma^{c, n+2} \zeta+\theta^{c a b} \kappa_{a b}, \\
& \zeta_{t}=-\frac{n}{4} \tau_{t t}+\gamma^{n+2,0} \tau_{t}+\gamma^{n+2,1} \tau+\gamma^{n+2, a+1} \chi^{a}+\gamma^{n+2, n+2} \zeta+\theta^{n+2, a b} \kappa_{a b}, \\
& \sigma_{t}=\gamma^{n+1,0} \tau_{t}+\gamma^{n+1,1} \tau+\gamma^{n+1, a+1} \chi^{a}+\gamma^{n+1, n+2} \zeta+\theta^{n+1, a b} \kappa_{a b},
\end{aligned}
$$

where the coefficients $\gamma^{p q}$ and $\theta^{p a b}, p=0, \ldots, n+2, q=0, \ldots, n+2, a<b$, are functions of $t$. From this it is clear that the upper bound of $\operatorname{dim} \mathfrak{g}_{S}$ can not exceed the sum of the number of pairs $(a, b)$ of rotations with $a<b$ and the number of arbitrary constants involved in the general solution of the above system, i.e., $n(n+3) / 2+5$. At the same time, Lemma 16 shows that this number is reduced by 1 for any $S$ with $\left(\rho S_{\rho}\right)_{\rho} \neq 0$ or with $S_{a \rho} \neq 0$ for some $a$. For other values of $S$, we differentiate the classifying condition (15) with respect to $\rho$. In view of the constraint $S_{\rho} \neq 0$, the obtained equation $S_{\rho} \tau_{t}+S_{t \rho} \tau=0$ implies a linear first-order ordinary differential equation in $\tau$, and thus the above number is reduced by 2 .

The dimension of $\mathfrak{g}_{S}$ for $S=\rho^{4 / n}$ coincides with the found upper bound.

Lemma 19. $\operatorname{dim} \mathfrak{g}_{S} \cap\langle P(\chi), \sigma M\rangle \leqslant 2 n+1$ for any $S$ with $S_{\rho} \neq 0$.

Proof. Modifying the proof of Lemma 18, we omit the first and penultimate equations of the system from that proof and set $\tau=0, \kappa_{a b}=0$ and $\zeta=0$.

\section{Schrödinger equations with potentials and modular nonlinearity}

The class $\mathcal{V}$ of multidimensional nonlinear Schrödinger equations with potentials and modular nonlinearities of the form (5) can be embedded into the class $\mathcal{S}$ as the subclass $\tilde{\mathcal{V}}$ of equations with $S=f(\rho)+V(t, x)$, where $f$ is an arbitrary complex-valued nonlinearity depending only on $\rho:=|\psi|$ with $f_{\rho} \neq 0$ and $V$ is an arbitrary smooth complex-valued potential depending on $t$ and $x$. The subclass $\tilde{\mathcal{V}}$ is singled out from the class $\mathcal{S}$ by the constraints $S_{\rho t}=S_{\rho a}=0$ and $S_{\rho} \neq 0$ or, equivalently,

$$
\psi S_{\psi t}+\psi^{*} S_{\psi^{*} t}=\psi S_{\psi a}+\psi^{*} S_{\psi^{*} a}=0, \quad \psi S_{\psi}+\psi^{*} S_{\psi^{*}} \neq 0 .
$$

Equivalence transformations of the subclass $\tilde{\mathcal{V}}$ are exhausted by the elements of the group $G_{\tilde{\delta}}$ that preserve, in addition to (4) and (11), the constraints (16). It is obvious from Theorem 10 that not all admissible transformations of the subclass $\tilde{V}$ are generated by its equivalence transformations. 
Proposition 20. The class $\tilde{\mathcal{V}}$ is not normalized. The equivalence group $G_{\tilde{\mathcal{V}}}^{\tilde{v}}$ of this class consists of the point transformations (12) with $T_{t t}=0$ and $Z_{t}=0$.

The class $\mathcal{V}$ can be interpreted as a reparameterization of the class $\tilde{\mathcal{V}}$, where the parameter functions $f$ and $V$ are assumed to be arbitrary elements instead of $S$.

Proposition 21. The class $\mathcal{V}$ is not normalized. The equivalence group $G_{\mathcal{V}}$ of this class consists of the point transformations in the space with the coordinates $\left(t, x, \psi, \psi^{*}, f, f^{*}, V, V^{*}\right)$ whose $(t, x, \psi)$-components are of the form $(12 \mathrm{a})-(12 \mathrm{~b})$ and whose components for the arbitrary elements $f$ and $V$ are

$$
\tilde{f}=\frac{\hat{f}}{\left|T_{t}\right|}+c, \quad \tilde{V}=\frac{\hat{V}}{\left|T_{t}\right|}+\frac{\mathcal{X}_{t t}^{b}}{2\left|T_{t}\right|^{3 / 2}} O^{b a} x_{a}+\frac{\Sigma_{t}}{T_{t}}-\frac{\mathcal{X}_{t}^{a} \mathcal{X}_{t}^{a}}{4 T_{t}^{2}}-c,
$$

where $T, \mathcal{X}^{a}$ and $\Sigma$ are arbitrary smooth real-valued functions of $t$ with $T_{t} \neq 0$ and $T_{t t}=0$, $Z$ is an arbitrary real constant, $\varepsilon^{\prime}=\operatorname{sgn} T_{t}, O=\left(O^{a b}\right)$ is an arbitrary constant orthogonal $n \times n$ matrix, and $c$ is an arbitrary complex constant.

The appearance of the gauge equivalence transformations, which are associated with the group parameter $c$, is related to the ambiguity in the representation of $S$ as the sum of $f$ and $V$.

Generalizing Theorem 6 from [56] to an arbitrary $n \in \mathbb{N}$, we obtain the following assertion, which is motivated by Corollary 11 .

Theorem 22. The class $\mathcal{V}$ is partitioned into the normalized subclasses $\mathcal{V}^{\prime}$ and $\mathcal{P}_{\lambda}, \lambda \in \mathbb{R}$, which are singled out by the conditions that the ratio $\rho f_{\rho \rho} / f_{\rho}\left(\equiv \rho S_{\rho \rho} / S_{\rho}\right)$ is not a real constant and that $\rho f_{\rho \rho} / f_{\rho}=\lambda-1$, respectively, $\mathcal{V}=\mathcal{V}^{\prime} \sqcup\left(\bigsqcup_{\lambda \in \mathbb{R}} \mathcal{P}_{\lambda}\right)$. There are no point transformations relating equations from different subclasses among the above ones.

In other words, the above partition of the class $\mathcal{V}$ induces the partition of its equivalence groupoid $\mathcal{G}_{\mathcal{V}}=\mathcal{G}_{\mathcal{V}^{\prime}} \sqcup\left(\bigsqcup_{\lambda \in \mathbb{R}} \mathcal{G}_{\mathcal{P}_{\lambda}}\right)$, where the equivalence groupoids $\mathcal{G}_{\mathcal{V}^{\prime}}$ and $\mathcal{G}_{\mathcal{P}_{\lambda}}$ of the subclasses $\mathcal{V}^{\prime}$ and $\mathcal{P}_{\lambda}, \lambda \in \mathbb{R}$, are generated by the corresponding equivalence groups $G_{\mathcal{V}^{\prime}}$ and $G_{\mathcal{P}_{\lambda}}$. Moreover, $G_{\tilde{\mathcal{V}}^{\prime}}=G_{\mathcal{V}}$ and $G_{\mathcal{\mathcal { P }}_{\lambda}}^{\sim} \supsetneq G_{\mathcal{V}}$ for any $\lambda \in \mathbb{R}$, cf. the results of Sections 6.1-6.3 below. Therefore, a complete list of $\mathcal{G}_{\mathcal{v}}^{\lambda}$-inequivalent Lie-symmetry extensions in the class $\mathcal{V}$ is exhausted by the union of group-classification lists for the classes $\mathcal{V}^{\prime}$ and $\mathcal{P}_{\lambda}, \lambda \in \mathbb{R}$, with respect to the corresponding equivalence groups.

Remark 23. Instead of the partition of the class $\mathcal{V}$, one can consider the associated partition of the class $\tilde{\mathcal{V}}$, where the partition subclasses are singled out by the conditions that the ratio $\rho S_{\rho \rho} / S_{\rho}$ is not a real constant and that $\rho S_{\rho \rho} / S_{\rho}=\lambda-1, \lambda \in \mathbb{R}$, respectively. At the same time, one needs to handle these conditions jointly with conditions $S_{\rho t}=S_{\rho a}=0$ and $S_{\rho} \neq 0$ for the class $\mathcal{S}$. This is why it is more convenient to work with the reparameterized class $\mathcal{V}$ in spite of the fact that this class possesses gauge equivalence transformations.

Up to gauge equivalence transformations of the class $\mathcal{V}$, for equations from the subclasses $\mathcal{P}_{0}$ and $\mathcal{P}_{\lambda}$ with $\lambda \in \mathbb{R} \backslash\{0\}$ the arbitrary element $f$ takes the form $f=\delta \ln \rho$ and $f=\delta \rho^{\lambda}$, respectively, where $\delta$ is an arbitrary nonzero complex constant. We reparameterize these classes, assuming $\delta$ as an arbitrary element instead of $f$ and preserving the notation for these classes. Then gauge equivalence transformations are neglected.

\subsection{General case of modular nonlinearity}

As discussed above, the class $\mathcal{V}^{\prime}$ consists of the equations of the form

$$
i \psi_{t}+\psi_{a a}+f(\rho) \psi+V(t, x) \psi=0 \quad \text { with } \quad f_{\rho} \neq 0, \quad \rho f_{\rho \rho} / f_{\rho} \neq \text { const } \in \mathbb{R} .
$$

Proposition 21 implies the following assertion. 
Corollary 24. An equation from the class $\mathcal{V}^{\prime}$ with a potential $V$ is reduced by a point transformation to an equation from the same class with the zero potential if and only if the potential $V$ is real-valued and affine in $x$.

Remark 25. The action of each element of the group $G_{\mathcal{V}}$ on $f$ is the composition of complex conjugation, multiplication by a nonzero real constant, shift in a complex constant and rescaling its argument, which does not change the form of $f$ essentially. This is why in the course of group classification of the class $\mathcal{V}^{\prime}$, we can assume that the nonlinearity $f$ is fixed and the only arbitrary element is $V$. By $\nu^{f}$ we denote the subclass of equations in $\mathcal{V}^{\prime}$ with a fixed value of the arbitrary element $f . \mathcal{V}^{f}=\mathcal{V}^{\tilde{f}}$ if and only if $\tilde{f}-f=$ const $\in \mathbb{C}$. Hence the class $\mathcal{V}^{\prime}$ can be interpreted as the disjoint union of its subclasses $\mathcal{V}^{f}$, where $f$ runs through the set of complex-valued smooth functions of $\rho:=|\psi|$ with $f_{\rho} \neq 0$ and $\rho f_{\rho \rho} / f_{\rho} \neq$ const $\in \mathbb{R}$ modulo adding complex constants.

Proposition 26. The class $\mathcal{V}^{f}$ is normalized. Its equivalence group $G_{\mathcal{V} f}^{\sim}$ is constituted by the projections of elements of $G_{\mathcal{V}}$ on the space with the coordinates $\left(t, x, \psi, \psi^{*}, V, V^{*}\right)$, where $T_{t}=1$ (resp. $T_{t}= \pm 1$ if $f$ is a real-valued function), $Z=0$ and $c=0$.

Lemma 27. The maximal Lie invariance algebra $\mathfrak{g}_{V}$ of an equation $\mathcal{L}_{V}$ from $\mathcal{V}^{f}$ with $\rho f_{\rho \rho} / f_{\rho}$ not being a real constant consists of the vector fields of the form $D(c)-\sum_{a<b} \kappa_{a b} J_{a b}+P(\chi)+\sigma M$, where $c$ is an arbitrary real constant, $\left(\kappa_{a b}\right)$ is an arbitrary constant skew-symmetric $n \times n$ matrix and the parameter functions $\chi^{a}$ and $\sigma$ are arbitrary real-valued smooth functions of that satisfy the condition

$$
c V_{t}+\left(\kappa_{a b} x_{b}+\chi^{a}\right) V_{a}=\frac{1}{2} \chi_{t t}^{a} x_{a}+\sigma_{t} .
$$

The kernel Lie invariance algebra of equations from the class $\mathcal{V}^{f}$ is $\mathfrak{g}_{v_{f}}^{\cap}=\langle M\rangle$.

Proof. Substituting $S=f(\rho)+V(t, x)$ into the classifying condition (15) and recalling that $\rho f_{\rho \rho} / f_{\rho} \neq$ const $\in \mathbb{R}$, we derive $\tau_{t}=0$ and $\zeta=0$. The algebra $\mathfrak{g}_{v f}^{\cap}$ is obtained by varying the arbitrary element $V$ and splitting with respect to its derivatives.

Any vector field of the general form presented in Lemma 27, where at least one of the parameters $c, \kappa_{a b}$ and $\chi^{a}$ takes a nonzero value, belongs to $\mathfrak{g}_{V}$ for a potential $V$ satisfying the classifying condition (18) for this vector field. This is why we have

$$
\mathfrak{g}_{\langle\rangle}:=\sum_{V} \mathfrak{g}_{V}=\left\langle D(1), J_{a b}, a<b, P(\chi), \sigma M\right\rangle,
$$

where the parameter functions $\chi^{a}$ and $\sigma$ run through the set of real-valued smooth functions of $t$. This linear span is closed with respect to the Lie bracket of vector fields and thus is a Lie algebra, which coincides, in view of Proposition 26, with the projection of the equivalence algebra of the class $\mathcal{V}^{f}$ to the space with the coordinates $\left(t, x, \psi, \psi^{*}\right)$. Obtaining the above coincidence is the main incentive for fixing $f$. As a result, the action of the group $\pi_{*} G_{\mathcal{v} f}^{\sim}$ on the algebra $\mathfrak{g}_{\langle>}$is naturally consistent with this algebra. Therefore, within the framework of the algebraic method, the group classification of the class $\mathcal{V}^{f}$ reduces to the classification of appropriate subalgebras of $\mathfrak{g}_{\langle>}$up to $\pi_{*} G_{\mathcal{v} f}^{\sim}$-equivalence. The following conditions hold for any equation $\mathcal{L}_{V}$ from the class $\mathcal{V}^{f}$, and thus for any appropriate subalgebra of $\mathfrak{g}_{\langle\rangle}$, which by Definition 15 coincides with $\mathfrak{g}_{V}$ for some $V$ :

$$
\operatorname{dim} \mathfrak{g}_{V} \leqslant \frac{n(n+3)}{2}+2, \quad \operatorname{dim} \mathfrak{g}_{V} \cap\langle P(\chi), \sigma M\rangle \leqslant 2 n+1, \quad \mathfrak{g}_{V} \cap\langle\sigma M\rangle=\langle M\rangle .
$$




\subsection{Logarithmic modular nonlinearity}

The class $\mathcal{P}_{0}$ consists of the equations of the form

$$
i \psi_{t}+\psi_{a a}+\delta \psi \ln \rho+V(t, x) \psi=0,
$$

where $\delta$ is an arbitrary nonzero complex number, $\delta=\delta_{1}+i \delta_{2}, \delta_{1}, \delta_{2} \in \mathbb{R}$, and $V$ is an arbitrary complex-valued function of $t$ and $x$. The corresponding subclass of the class $\tilde{\mathcal{V}}$ is singled out from the class $\mathcal{S}$ by the constraints $S_{\rho t}=S_{\rho a}=0$ and $\left(\rho S_{\rho}\right)_{\rho}=0$, i.e., $\psi S_{\psi t}+\psi^{*} S_{\psi^{*} t}=$ $\psi S_{\psi a}+\psi^{*} S_{\psi^{*} a}=0$ and $\left(\psi \partial_{\psi}+\psi^{*} \partial_{\psi^{*}}\right)^{2} S=0$.

We can find the point transformations connecting two equations from the class $\mathcal{P}_{0}$ by the direct method. However, we have already described the equivalence groupoid $\mathcal{G}_{\mathcal{S}}^{\sim}$ of the class $\mathcal{S}$ in Theorem 10, so we can use this description and single out the equivalence groupoid of the class $\mathcal{P}_{0}$ as a subgroupoid of $\mathcal{G}_{\mathcal{S}}^{\sim}$, substituting $S=\delta \ln \rho+V(t, x)$ and $\tilde{S}=\tilde{\delta} \ln \tilde{\rho}+\tilde{V}(\tilde{t}, \tilde{x})$ into (12c).

Theorem 28. The class $\mathcal{P}_{0}$ is normalized. Its equivalence group $G_{\mathcal{P}_{0}}^{\sim}$ is constituted by the point transformations in the space with the coordinates $\left(t, x, \psi, \psi^{*}, \delta, \delta^{*}, V, V^{*}\right)$ whose components for the variables are of the form $(12 \mathrm{a})-(12 \mathrm{~b})$ and whose components for the arbitrary elements $\delta$ and $V$ are

$$
\tilde{\delta}=\frac{\hat{\delta}}{\left|T_{t}\right|}, \quad \tilde{V}=\frac{\hat{V}}{\left|T_{t}\right|}+\frac{\mathcal{X}_{t t}^{b}}{2\left|T_{t}\right|^{3 / 2}} O^{b a} x_{a}-\hat{\delta} \frac{Z}{\left|T_{t}\right|}-\frac{1}{4} \frac{\mathcal{X}_{t}^{a} \mathcal{X}_{t}^{a}}{T_{t}^{2}}+\frac{\Sigma_{t}-i Z_{t}}{T_{t}},
$$

where the parameter functions $T, \mathcal{X}^{a}, Z$ and $\Sigma$ are arbitrary smooth real-valued functions of $t$ with $T_{t} \neq 0$ and $T_{t t}=0, \varepsilon^{\prime}=\operatorname{sgn} T_{t}$ and $O=\left(O^{a b}\right)$ is an arbitrary constant orthogonal $n \times n$ matrix.

Corollary 29. An equation from the class $\mathcal{P}_{0}$ with a potential $V$ is reduced by a point transformation to an equation from the same class with the zero potential if and only if the potential $V$ is affine in $x$ and the coefficients of $x_{a}$ are real-valued, i.e., $V_{a b}=0$ and $V_{a}$ are real-valued.

Remark 30. Theorem 28 implies that any point transformation connecting two equations in the class $\mathcal{P}_{0}$ acts on $\delta$ by multiplication with a nonzero real constant and by complex conjugation. Similarly to Remark 25, we can fix an arbitrary value of $\delta$ and assume $V$ the only arbitrary element. By $\mathcal{P}_{0}^{\delta}$ we denote the subclass of equations in $\mathcal{P}_{0}$ with a fixed value of $\delta$. The class $\mathcal{P}_{0}$ can be interpreted as the disjoint union of its subclasses $\mathcal{P}_{0}^{\delta}$, where $\delta$ runs through the set of nonzero complex numbers. Theorem 28 implies that equations from subclasses $\mathcal{P}_{0}^{\delta}$ and $\mathcal{P}_{0}^{\tilde{\delta}}$ are related by point transformations if and only if $\tilde{\delta} / \delta \in \mathbb{R}_{>0}$ or $\tilde{\delta} / \delta^{*} \in \mathbb{R}_{>0}$, and then the point transformation $\mathcal{D}(T)$ with $T=\delta \tilde{\delta}^{-1} t$ or with $T=-\delta^{*} \tilde{\delta}^{-1} t$, respectively, maps the entire subclass $\mathcal{P}_{0}^{\delta}$ onto the subclass $\mathcal{P}_{0}^{\tilde{\delta}}$. In other words, up to $G_{\mathcal{P}_{0}}^{\sim}$-equivalence one can set the constraints $|\delta|=1$ and $\operatorname{Im} \delta \geqslant 0$ on $\delta$.

Proposition 31. The class $\mathcal{P}_{0}^{\delta}$ is normalized. The equivalence group $G_{\mathcal{P}^{\delta}}^{\sim}$ of this class is constituted by the projections of elements of $G_{\mathcal{P}_{0}}^{\sim}$ on the space with the coordinates $\left(t, x, \psi, \psi^{*}, V, V^{*}\right)$, where $T_{t}=1$ if $\delta_{2}:=\operatorname{Im} \delta \neq 0$ and $T_{t}= \pm 1$ if $\delta_{2}=0$.

Setting $S=\delta \ln \rho+V(t, x)$ in the classifying condition (15) and splitting with respect to $\rho$ yield $\tau_{t}=0$.

Lemma 32. Any vector field $Q$ from the maximal Lie invariance algebra $\mathfrak{g}_{V}$ of an equation $\mathcal{L}_{V}$ from the subclass $\mathcal{P}_{0}^{\delta}$ is of the form $D(c)-\sum_{a<b} \kappa_{a b} J_{a b}+P(\chi)+\sigma M+\zeta I$, where $c$ is an arbitrary real constant, $\left(\kappa_{a b}\right)$ is an arbitrary constant skew-symmetric $n \times n$ matrix and the parameter functions $\chi^{a}, \sigma$ and $\zeta$ are arbitrary real-valued smooth functions of t that satisfy the classifying condition

$$
c V_{t}+\left(\kappa_{a b} x_{b}+\chi^{a}\right) V_{a}=\frac{1}{2} \chi_{t t}^{a} x_{a}+\sigma_{t}-i \zeta_{t}-\delta \zeta .
$$


Lemma 33. The kernel Lie invariance algebra of equations from the class $\mathcal{P}_{0}^{\delta}$ is $\mathfrak{g}_{\mathcal{P}^{\delta}}^{\cap}=\left\langle M, I^{\prime}\right\rangle$, where $I^{\prime}=e^{-\delta_{2} t}\left(\delta_{2} I-\delta_{1} M\right)$ if $\delta_{2} \neq 0$ and $I^{\prime}=I+\delta_{1} t M$ if $\delta_{2}=0$. The kernel point-symmetry group of this class consists of the transformations of the form (12a)-(12b) with $T=t, \mathcal{X}=0$, $O=E, Z=Z_{0} \delta_{2} e^{-\delta_{2} t}, \Sigma=\Sigma_{0}-Z_{0} \delta_{1} e^{-\delta_{2} t}$ if $\delta_{2} \neq 0$ and $Z=Z_{0}, \Sigma=\Sigma_{0}+Z_{0} \delta_{1} t$ if $\delta_{2}=0$, where $\Sigma_{0}$ and $Z_{0}$ are arbitrary real constants.

Any vector field of the general form from Lemma 32, where at least one of the parameters $c, \kappa_{a b}$ and $\chi^{a}$ takes a nonzero value, belongs to $\mathfrak{g}_{V}$ for a potential $V$ satisfying the classifying condition (21) for this vector field. This is why we have

$$
\mathfrak{g}_{\langle\rangle}:=\sum_{V} \mathfrak{g}_{V}=\left\langle D(1), J_{a b}, a<b, P(\chi), \sigma M, \zeta I\right\rangle,
$$

where the parameter functions $\chi^{a}, \sigma$ and $\zeta$ run through the set of real-valued smooth functions of $t$. This linear span is closed with respect to the Lie bracket of vector fields and thus is a Lie algebra, which coincides, in view of Proposition 31, with the projection of the equivalence algebra of the class $\mathcal{P}_{0}^{\delta}$ to the space with the coordinates $\left(t, x, \psi, \psi^{*}\right)$. The above coincidence obtained due to fixing $\delta$ leads to the consistency of the action of the group $\pi_{*} G_{\mathcal{P} \delta}^{\sim}$ with the algebra $\mathfrak{g}_{\langle\rangle}$. Therefore, within the framework of the algebraic method, the group classification of the class $\mathcal{P}_{0}^{\delta}$ reduces to the classification of appropriate subalgebras of $\mathfrak{g}_{\langle\rangle}$up to $\pi_{*} G_{\mathcal{P}_{\hat{\beta}}^{-}}^{\sim}$-equivalence. The following conditions hold for any equation $\mathcal{L}_{V}$ from the class $\mathcal{P}_{0}^{\delta}$, and thus for any appropriate subalgebra of $\mathfrak{g}_{\langle\rangle}$, which by Definition 15 coincides with $\mathfrak{g}_{V}$ for some $V$ :

$$
\begin{aligned}
& \operatorname{dim} \mathfrak{g}_{V} \leqslant \frac{n(n+3)}{2}+3, \quad \operatorname{dim} \mathfrak{g}_{V} \cap\langle P(\chi), \sigma M, \zeta I\rangle \leqslant 2 n+2, \\
& \mathfrak{g}_{V} \cap\langle\sigma M, \zeta I\rangle=\left\langle M, I^{\prime}\right\rangle=\mathfrak{g}_{\mathcal{P}_{0}^{\delta}}^{\cap} .
\end{aligned}
$$

\subsection{Power modular nonlinearity}

For any fixed nonzero real constant $\lambda$, the class $\mathcal{P}_{\lambda}$ of nonlinear Schrödinger equations with potentials and power nonlinearity consists of the equations of the form

$$
i \psi_{t}+\psi_{a a}+\delta \rho^{\lambda} \psi+V(t, x) \psi=0 .
$$

Here $\delta$ is an arbitrary nonzero complex constant and $V$ is an arbitrary complex-valued potential depending on $t$ and $x$. The subclass of the class $\tilde{\mathcal{V}}$ corresponding to the class $\mathcal{P}_{\lambda}$ is singled out from the class $\mathcal{S}$ by the constraints $S_{\rho t}=S_{\rho a}=0,\left(\rho S_{\rho}\right)_{\rho}=\lambda S_{\rho}$ or, equivalently, by the constraints

$$
\psi S_{\psi t}+\psi^{*} S_{\psi^{*} t}=\psi S_{\psi a}+\psi^{*} S_{\psi^{*} a}=0, \quad\left(\psi \partial_{\psi}+\psi^{*} \partial_{\psi^{*}}\right)^{2} S=\lambda\left(\psi \partial_{\psi}+\psi^{*} \partial_{\psi^{*}}\right) S .
$$

Using the description of the equivalence groupoid $\mathcal{G}_{\mathcal{S}}^{\sim}$ of the class $\mathcal{S}$ in Theorem 10, we single out the equivalence groupoid of the class $\mathcal{P}_{\lambda}$ as a subgroupoid of $\mathcal{G}_{\mathcal{S}}^{\sim}$, substituting $S=$ $\delta \rho^{\lambda}+V(t, x)$ and $\tilde{S}=\tilde{\delta} \tilde{\rho}^{\lambda}+\tilde{V}(\tilde{t}, \tilde{x})$ into $(12 \mathrm{c})$.

Theorem 34. The class $\mathcal{P}_{\lambda}$ with $\lambda \in \mathbb{R}_{\neq 0}$ is normalized. Its equivalence group $G_{\tilde{\mathcal{P}}_{\lambda}}$ consists of the point transformations in the space with the coordinates $\left(t, x, \psi, \psi^{*}, \delta, \delta^{*}, V, V^{*}\right)$ whose $(t, x, \psi)$-components are of the form (12a)-(12b) and whose components for the arbitrary elements $\delta$ and $V$ are

$$
\tilde{\delta}=\frac{\hat{\delta}}{\mu^{\lambda}}, \quad \tilde{V}=\frac{\hat{V}}{\left|T_{t}\right|}+\frac{2 T_{t t t} T_{t}-3 T_{t t}^{2}}{16 \varepsilon^{\prime} T_{t}^{3}} x_{a} x_{a}+\frac{\varepsilon^{\prime}}{2}\left(\frac{\mathcal{X}_{t}^{b}}{T_{t}}\right)_{t} \frac{O^{b a} x_{a}}{\left|T_{t}\right|^{1 / 2}}+\frac{\Sigma_{t}}{T_{t}}-\frac{\mathcal{X}_{t}^{a} \mathcal{X}_{t}^{a}}{4 T_{t}^{2}}+i \lambda^{\prime} \frac{T_{t t}}{T_{t}^{2}},
$$

where $\lambda^{\prime}:=1 / \lambda-n / 4, T, \mathcal{X}^{a}$ and $\Sigma$ are real-valued functions of $t$ with $T_{t} \neq 0$, and $e^{Z}=\mu\left|T_{t}\right|^{-1 / \lambda}$ with real constant $\mu>0$. 
Corollary 35. A $(1+n)$-dimensional nonlinear Schrödinger equation of the form (22) with a potential $V$ is reduced by a point transformation to an equation of the same form with potential independent of $x$ if and only if

$$
V=h(t) x_{a} x_{a}+h^{a}(t) x_{a}+\tilde{h}^{0}(t)+i h^{0}(t)
$$

where $h, h^{a}, h^{0}$ and $\tilde{h}^{0}$ are real-valued functions of $t$. Moreover, the transformed potential can be assumed imaginary-valued.

Corollary 36. $A(1+n)$-dimensional nonlinear Schrödinger equation of the form (22) with a potential $V$ is reduced by a point transformation to an equation of the same form with zero potential if and only if $V$ is of the form (24), where $16\left(\lambda^{\prime}\right)^{2} h=2 \lambda^{\prime} h_{t}^{0}+\left(h^{0}\right)^{2}$. If $\lambda=4 / n$, then the condition for $V$ means that $V$ is an arbitrary real-valued $x$-quadratic potential.

Proof. The expression (24) for $V$ that is reducible to zero is obtained by setting $\tilde{V}=0$ in Theorem 34. The representations

$$
h^{0}=-\lambda^{\prime} \frac{T_{t t}}{T_{t}}, \quad h=-\frac{2 T_{t t t} T_{t}-3 T_{t t}^{2}}{16 T_{t}^{2}}
$$

are jointly equivalent to the equality $16\left(\lambda^{\prime}\right)^{2} h=2 \lambda^{\prime} h_{t}^{0}+\left(h^{0}\right)^{2}$.

Remark 37. Instead of the family of the subclasses $\mathcal{P}_{\lambda}$ parameterized by $\lambda \in \mathbb{R}_{\neq 0}$, one can study the entire class $\mathcal{P}$ of $(1+n)$-dimensional nonlinear Schrödinger equations with potentials and power modular nonlinearities, i.e., the class of equations of the form (22), where $\lambda$ is assumed to be one more constant arbitrary element running through $\mathbb{R}_{\neq 0}$. We can interpret the class $\mathcal{P}$ as the disjoint union of the subclasses $\mathcal{P}_{\lambda}$ with $\lambda \in \mathbb{R}_{\neq 0}$. A drawback of the above approach is the need to consider the generalized equivalence group of $\mathcal{P}$, where the $x$-component of equivalence transformations will depend on $\lambda$, see Theorem 34, since the class $\mathcal{P}$ is normalized in the generalized sense only.

Remark 38. Arguing similarly to Remark 30 using Theorem 34, we fix an arbitrary value of $\delta$ and assume $V$ the only arbitrary element. By $\mathcal{P}_{\lambda}^{\delta}$ we denote the subclass of equations in $\mathcal{P}_{\lambda}$ with a fixed value of $\delta$. The class $\mathcal{P}_{\lambda}$ can be interpreted as the disjoint union of its subclasses $\mathcal{P}_{\lambda}^{\delta}$, where $\delta$ runs through the set of nonzero complex numbers. Theorem 34 implies that equations from subclasses $\mathcal{P}_{\lambda}^{\delta}$ and $\mathcal{P}_{\lambda}^{\tilde{\delta}}$ are related by point transformations if and only if $\tilde{\delta} / \delta \in \mathbb{R}_{>0}$ or $\tilde{\delta} / \delta^{*} \in \mathbb{R}_{>0}$, and then the point transformation $\mathcal{D}(T)$ with $T=\delta \tilde{\delta}^{-1} t$ or with $T=-\delta^{*} \tilde{\delta}^{-1} t$, respectively, maps the entire subclass $\mathcal{P}_{0}^{\delta}$ onto the subclass $\mathcal{P}_{0}^{\tilde{\delta}}$. In other words, up to $G_{\widetilde{\mathcal{P}}_{0}}^{\sim}$-equivalence one can set the constraints $|\delta|=1$ and $\operatorname{Im} \delta \geqslant 0$ on $\delta$.

Proposition 39. The class $\mathcal{P}_{\lambda}^{\delta}$ is normalized. The equivalence group $G_{\mathcal{P}^{\delta}}^{\sim}$ of this class is constituted by the projections of elements of $G_{\mathcal{P}_{\lambda}}^{\sim}$ onto the space with the coordinates $\left(t, x, \psi, \psi^{*}, V, V^{*}\right)$, where $\mu=1$ and, if $\operatorname{Im} \delta \neq 0, T_{t}>0$.

Substituting $S=\delta \rho^{\lambda}+V(t, x)$ into the classifying condition (15) and splitting with respect to $\rho$, we obtain the determining equation $\lambda \zeta+\tau_{t}=0$ and the classifying condition for the class $\mathcal{P}_{\lambda}^{\delta}$,

$$
\tau V_{t}+\left(\frac{1}{2} \tau_{t} x_{a}+\kappa_{a b} x_{b}+\chi^{a}\right) V_{a}+\tau_{t} V=\frac{1}{8} \tau_{t t t} x_{a} x_{a}+\frac{1}{2} \chi_{t t}^{a} x_{a}+\sigma_{t}+i \lambda^{\prime} \tau_{t t} .
$$

As a result, Theorem 13 implies the following assertion.

Lemma 40. Any vector field $Q$ from the maximal Lie invariance algebra $\mathfrak{g}_{V}$ of an equation $\mathcal{L}_{V}$ from the class $\mathcal{P}_{\lambda}^{\delta}$ is of the form $D^{\lambda}(\tau)-\sum_{a<b} \kappa_{a b} J_{a b}+P(\chi)+\sigma M$, where $D^{\lambda}(\tau)=D(\tau)-\lambda^{-1} \tau_{t} I$, $\left(\kappa_{a b}\right)$ is an arbitrary constant skew-symmetric $n \times n$ matrix, and the parameter functions $\tau, \chi^{a}$ and $\sigma$ are arbitrary real-valued smooth functions of $t$ that satisfy the classifying condition (25). 
Lemma 41. The kernel Lie invariance algebra of the equations from the class $\mathcal{P}_{\lambda}^{\delta}$ is $\mathfrak{g}_{\mathcal{P}_{\lambda}^{\delta}}^{\cap}=\langle M\rangle$.

Any vector field of the general form from Lemma 40, where at least one of the parameters $\tau$, $\kappa_{a b}, \chi^{a}$ and $\sigma$ takes a nonzero value, belongs to $\mathfrak{g}_{V}$ for a potential $V$ satisfying the classifying condition (25) for this vector field. This is why we have

$$
\mathfrak{g}_{\langle\rangle}:=\sum_{V} \mathfrak{g}_{V}=\left\langle D^{\lambda}(\tau), J_{a b}, a<b, P(\chi), \sigma M\right\rangle,
$$

where the parameter functions $\tau, \chi^{a}$ and $\sigma$ run through the set of real-valued smooth functions of $t$. This linear span is closed with respect to the Lie bracket of vector fields and thus is a Lie algebra, which coincides, in view of Proposition 39, with the projection of the equivalence algebra of the class $\mathcal{P}_{\lambda}^{\delta}$ to the space with the coordinates $\left(t, x, \psi, \psi^{*}\right)$. The above coincidence is again both a consequence of and a justification for fixing $\delta$ since the action of the group $\pi_{*} G_{\mathcal{P}^{\delta}}^{\sim}$ is then consistent with the algebra $\mathfrak{g}_{\langle\rangle}$. Therefore, within the framework of the algebraic method, the group classification of the class $\mathcal{P}_{\lambda}^{\delta}$ reduces to the classification of the appropriate subalgebras of $\mathfrak{g}_{\langle\rangle}$up to $\pi_{*} G_{\mathcal{P}^{\delta}}^{\sim}$-equivalence. The following conditions hold for any equation $\mathcal{L}_{V}$ from the class $\mathcal{P}_{\lambda}^{\delta}$, and thus for any appropriate subalgebra of $\mathfrak{g}_{\langle\rangle}$, which by Definition 15 coincides with $\mathfrak{g}_{V}$ for some $V$ :

$$
\operatorname{dim} \mathfrak{g}_{V} \leqslant \frac{n(n+3)}{2}+4, \quad \mathfrak{g}_{V} \cap\langle\sigma M\rangle=\langle M\rangle, \quad \operatorname{dim} \mathfrak{g}_{V} \cap\langle P(\chi), \sigma M\rangle \leqslant 2 n+1 .
$$

Lemma 42. For all $V, \pi_{*}^{0} \mathfrak{g}_{V}$ is a Lie algebra and $\operatorname{dim} \pi_{*}^{0} \mathfrak{g}_{V} \leqslant 3$. Moreover,

$$
\pi_{*}^{0} \mathfrak{g}_{V} \in\left\{\{0\},\left\langle\partial_{t}\right\rangle,\left\langle\partial_{t}, t \partial_{t}\right\rangle,\left\langle\partial_{t}, t \partial_{t}, t^{2} \partial_{t}\right\rangle\right\} \bmod \pi_{*}^{0} G_{\mathcal{P}_{\lambda}}, \quad \text { where } \quad \pi_{*}^{0} \mathfrak{g}_{\langle\rangle}=\left\langle\tau \partial_{t}\right\rangle,
$$

and $\pi^{0}$ is the projection onto the space with the coordinate $t$.

\section{Group classification in dimension $(1+2)$}

We present the complete group classification of the class $\mathcal{V}$ for $n=2$. We recall that this class is not normalized but it is partitioned into the normalized subclasses $\mathcal{V}^{\prime}, \mathcal{P}_{0}$ and $\mathcal{P}_{\lambda}, \lambda \in \mathbb{R}_{\neq 0}$, consisting of equations whose general forms are (17), (19) and (22), respectively. Following results of Section 5 , we consider the classes $\mathcal{V}^{f}, \mathcal{P}_{0}^{\delta}$ and $\mathcal{P}_{\lambda}^{\delta}$ instead of $\mathcal{V}^{\prime}, \mathcal{P}_{0}$ and $\mathcal{P}_{\lambda}$, respectively.

In this section, the maximal Lie invariance algebra of the equation from one of the classes under consideration with a potential $V$ and the corresponding nonlinearity is denoted by $\mathfrak{g}_{V}$. The indices $a$ and $b$ run from 1 to 2 , and we assume summation over repeated indices. Since here the collection $\left\{J_{a b}, a<b\right\}$ consists of the single vector field $J_{12}$, for convenience we re-denote $J:=J_{12}$ and $\kappa:=-\kappa_{12}=\kappa_{21}$. In each subsection of this section, we will omit the indication of the class under study in the notation of its equivalence group, shortly denoting this group by $G^{\sim}$. We also use the notations

$$
|x|=\sqrt{x_{1}^{2}+x_{2}^{2}}, \quad \phi=\arctan \frac{x_{2}}{x_{1}}, \quad \omega_{1}=x_{1} \cos \kappa t+x_{2} \sin \kappa t, \quad \omega_{2}=-x_{1} \sin \kappa t+x_{2} \cos \kappa t .
$$

For each appropriate subalgebra $\mathfrak{s}$ of the associated linear span $\mathfrak{g}_{\langle\rangle}$, we also introduce five integers that are invariant under equivalence transformations of the class under study. We define these integers as

$$
\begin{aligned}
& r_{1}:=\operatorname{rank}\{\chi \mid \exists \sigma: P(\chi)+\sigma M \in \mathfrak{s}\}, \\
& k_{0}:=\operatorname{dim} \mathfrak{s} \cap\langle\sigma M\rangle=\operatorname{dim} \mathfrak{g}^{\cap}=1 \quad \text { since } \mathfrak{s} \supseteq \mathfrak{g}^{\cap}=\langle M\rangle, \\
& k_{1}:=\operatorname{dim} \mathfrak{s} \cap\langle P(\chi), \sigma M\rangle-k_{0}, \\
& k_{2}:=\operatorname{dim} \mathfrak{s} \cap\langle J, P(\chi), \sigma M\rangle-k_{1}-k_{0}, \\
& k_{3}:=\operatorname{dim} \mathfrak{s}-k_{2}-k_{1}-k_{0}=\operatorname{dim} \pi_{*}^{0} \mathfrak{s}
\end{aligned}
$$


for the classes $\mathcal{V}^{f}$ and $\mathcal{P}_{0}^{\delta}$ in Sections 6.1 and 6.3 and as

$$
\begin{aligned}
& r_{1}:=\operatorname{rank}\{\chi \mid \exists \sigma, \zeta: P(\chi)+\sigma M+\zeta I \in \mathfrak{s}\}, \\
& k_{0}:=\operatorname{dim} \mathfrak{s} \cap\langle\sigma M, \zeta I\rangle=\operatorname{dim} \mathfrak{g}^{\cap}=2 \quad \text { since } \mathfrak{s} \supseteq \mathfrak{g}^{\cap}=\langle M, I\rangle, \\
& k_{1}:=\operatorname{dim} \mathfrak{s} \cap\langle P(\chi), \sigma M, \zeta I\rangle-k_{0}, \\
& k_{2}:=\operatorname{dim} \mathfrak{s} \cap\langle J, P(\chi), \sigma M, \zeta I\rangle-k_{1}-k_{0}, \\
& k_{3}:=\operatorname{dim} \mathfrak{s}-k_{2}-k_{1}-k_{0}=\operatorname{dim} \pi_{*}^{0} \mathfrak{s}
\end{aligned}
$$

for the class $\mathcal{P}_{\lambda}^{\delta}$ in Section 6.2. Here and in what follows $\pi^{0}$ is the projection onto the space of the variable $t$, and the parameter functions $\chi^{1}, \chi^{2}, \sigma$ and $\zeta$ in linear spans run through the set of real-valued smooth functions of $t$. It is obvious that in each case

$$
\operatorname{dim} \mathfrak{s}=k_{0}+k_{1}+k_{2}+k_{3}, \quad r_{1} \in\{0,1,2\}, \quad k_{2} \in\{0,1\}, \quad r_{1} \leqslant k_{1},
$$

and $r_{1}=0$ if and only if $k_{1}=0$. Results of Sections 4 and 5 imply that $k_{1} \in\{0, \ldots, 4\} ; k_{3} \in$ $\{0,1\}$ within the classes $\mathcal{V}^{f}$ and $\mathcal{P}_{0}^{\delta}$ and $k_{3} \in\{0,1,2,3\}$ within the class $\mathcal{P}_{\lambda}^{\delta}$, and $\operatorname{dim} \mathfrak{s} \leqslant 7,8,9$ for the classes $\mathcal{V}^{f}, \mathcal{P}_{0}^{\delta}$ and $\mathcal{P}_{\lambda}^{\delta}$, respectively.

Lemma 43. If $r_{1}=1$, then $k_{2}=0$.

Proof. Suppose that $r_{1}=1$ and $k_{2}=1$. Then the algebra $\mathfrak{g}_{V}$ contains vector fields $Q^{1}=$ $P\left(\chi^{11}, \chi^{12}\right)+Q^{10}$ with $\left(\chi^{11}, \chi^{12}\right) \neq(0,0)$ and $Q^{0}=J+P\left(\chi^{01}, \chi^{02}\right)+Q^{00}$. The condition $Q^{2}:=\left[Q^{0}, Q^{1}\right]=P\left(\chi^{12},-\chi^{11}\right)+Q^{20} \in \mathfrak{g}_{V}$ implies $r_{1}=2$, contradicting the condition $r_{1}=1$. Here $Q^{j 0} \in\langle\sigma M\rangle$ for the classes $\mathcal{V}^{f}$ and $\mathcal{P}_{0}^{\delta}$ and $Q^{j 0} \in\langle\sigma M, \zeta I\rangle$ for the class $\mathcal{P}_{\lambda}^{\delta}, j=0,1,2$.

Below we separately solve the group classification problems for each of the classes $\mathcal{V}^{f}, \mathcal{P}_{0}^{\delta}$ and $\mathcal{P}_{\lambda}^{\delta}, \lambda \in \mathbb{R}_{\neq 0}$. For this purpose, we classify appropriate subalgebras of the corresponding algebra $\mathfrak{g}_{\langle\rangle}$, taking into account the above constraints on the introduced invariant integers and on the structure of appropriate subalgebras in general as well as the classification conditions (18), (21) and (25) for the classes $\mathcal{V}^{f}, \mathcal{P}_{0}^{\delta}$ and $\mathcal{P}_{\lambda}^{\delta}$, respectively. Simultaneously or successively with this, we integrate the equations on $V$ implied by the corresponding classification condition for each appropriate subalgebra to be listed. In the course of solving the classification problems, we find more constraints for the invariant integers, whose derivation, in contrast to the presented constraints, is intricate for separating it from the process of group classification:

$k_{1} \neq 3 ; \quad r_{1}=1$ if and only if $k_{1} \in\{1,2\} ; \quad r_{1}=2$ if and only if $k_{1}=4$;

for the class $\mathcal{P}_{\lambda}^{\delta}: \quad k_{3} \in\{0,1,2\} \quad$ if $\lambda \neq 2, \quad k_{3} \in\{0,1,3\} \quad$ if $\lambda=2$.

\subsection{General case of modular nonlinearity}

In view of results of Section 5.1, the following conditions hold for any equation $\mathcal{L}_{V}$ from the class $\mathcal{V}^{f}$ with $n=2$ :

$$
\operatorname{dim} \mathfrak{g}_{V} \leqslant 7, \quad r_{1} \in\{0,1,2\}, \quad k_{0}=1, \quad k_{1} \in\{0, \ldots, 4\}, \quad k_{2} \in\{0,1\}, \quad k_{3} \in\{0,1\} .
$$

Therefore, any appropriate subalgebra of $\mathfrak{g}_{\langle\rangle}$is spanned by

- the basis vector field $M$ of the kernel $\mathfrak{g}^{\cap}$,

- $k_{1}$ vector fields $P\left(\chi^{p 1}, \chi^{p 2}\right)+\sigma^{p} M$ with linearly independent tuples $\left(\chi^{p 1}, \chi^{p 2}\right), p=1, \ldots, k_{1}$,

- $k_{2}$ vector fields of the form $J+P\left(\chi^{01}, \chi^{02}\right)+\sigma^{0} M$,

- $k_{3}$ vector fields of the form $D(1)+\kappa_{q} J+P\left(\chi^{q 1}, \chi^{q 2}\right)+\sigma^{q} M$ with $q=k_{1}+k_{3}$. 
Theorem 44. A complete list of inequivalent Lie symmetry extensions in the class $\mathcal{V}^{f}$ is exhausted by the cases listed below, where $U$ is an arbitrary complex-valued smooth function of its arguments or an arbitrary complex constant, and the other functions and constants take real values.

0. $V=V(t, x): \quad \mathfrak{g}_{V}=\mathfrak{g}^{\cap}=\langle M\rangle$.

1. $V=U\left(x_{1}, x_{2}\right): \mathfrak{g}_{V}=\langle M, D(1)\rangle$.

2. $V=U\left(\omega_{1}, \omega_{2}\right), \quad \kappa \neq 0: \quad \mathfrak{g}_{V}=\langle M, D(1)+\kappa J\rangle$.

3. $V=U(t,|x|)+\sigma_{t}(t) \phi: \quad \mathfrak{g}_{V}=\langle M, J+\sigma(t) M\rangle$.

4. $V=U(|x|)+\mu \phi: \quad \mathfrak{g}_{V}=\langle M, J+\mu t M, D(1)\rangle$.

5. $V=U\left(t, x_{2}\right)+\frac{1}{4} h^{11}(t) x_{1}^{2}: \quad \mathfrak{g}_{V}=\left\langle M, P\left(\theta^{1}, 0\right), P\left(\theta^{2}, 0\right)\right\rangle$, where $\theta^{1}$ and $\theta^{2}$ are linearly independent solutions of the equation $\theta_{t t}=h^{11} \theta$.

6. $V=U\left(x_{2}\right)+\frac{1}{4} \alpha x_{1}^{2}: \mathfrak{g}_{V}=\left\langle M, P\left(\theta^{1}, 0\right), P\left(\theta^{2}, 0\right), D(1)\right\rangle$, where $\theta^{1}$ and $\theta^{2}$ are linearly independent solutions of the equation $\theta_{t t}=\alpha \theta$.

7. $V=U(t, \varpi)+\frac{\chi_{t t}^{1}}{4 \chi^{1}} x_{1}^{2}+\frac{\chi_{t t}^{2}}{4 \chi^{2}} x_{2}^{2}, \chi^{1} \chi_{t}^{2}-\chi^{2} \chi_{t}^{1} \neq 0, \varpi=\chi^{1} x_{2}-\chi^{2} x_{1}: \quad \mathfrak{g}_{V}=\left\langle M, P\left(\chi^{1}, \chi^{2}\right)\right\rangle$.

8. $V=U\left(\omega_{2}\right)+\frac{1}{4}\left(\alpha^{2}-\kappa^{2}\right) \omega_{1}^{2}+\alpha \kappa \omega_{1} \omega_{2}, \alpha \kappa \neq 0$ : $\mathfrak{g}_{V}=\left\langle M, P\left(e^{\alpha t} \cos \kappa t, e^{\alpha t} \sin \kappa t\right), D(1)+\kappa J\right\rangle$.

9. $V=U\left(\omega_{2}\right)-\frac{1}{4} \kappa^{2} \omega_{1}^{2}+\mu \omega_{1}, \quad \kappa \neq 0, \mu \geqslant 0: \quad \mathfrak{g}_{V}=\langle M, P(\cos \kappa t, \sin \kappa t)+\mu t M, D(1)+\kappa J\rangle$.

10. $V=\frac{1}{4} h^{a b}(t) x_{a} x_{b}+i h^{00}(t), h^{12}=h^{21}: \mathfrak{g}_{V}=\left\langle M, P\left(\chi^{p 1}, \chi^{p 2}\right), p=1, \ldots, 4\right\rangle$, where $\left\{\left(\chi^{p 1}(t), \chi^{p 2}(t)\right)\right\}$ is a fundamental set of solutions of the system $\chi_{t t}^{a}=h^{a b} \chi^{b}$.

11. $V=\frac{1}{4} \alpha x_{1}^{2}+\frac{1}{4} \beta x_{2}^{2}+i \nu, \beta \neq \alpha \neq 0: \mathfrak{g}_{V}=\left\langle M, P\left(\theta^{1}, 0\right), P\left(\theta^{2}, 0\right), P\left(0, \theta^{3}\right), P\left(0, \theta^{4}\right), D(1)\right\rangle$, where $\left\{\theta^{1}(t), \theta^{2}(t)\right\}$ and $\left\{\theta^{3}(t), \theta^{4}(t)\right\}$ are fundamental sets of solutions of the equations $\theta_{t t}=\alpha \theta$ and $\theta_{t t}=\beta \theta$, respectively.

12. $V=\frac{1}{4} \alpha \omega_{1}^{2}+\frac{1}{4} \beta \omega_{2}^{2}+i \nu, \beta \neq \alpha \neq 0, \quad \kappa \neq 0$ :

$\mathfrak{g}_{V}=\left\langle M, P\left(\theta^{p 1} \cos \kappa t-\theta^{p 2} \sin \kappa t, \theta^{p 1} \sin \kappa t+\theta^{p 2} \cos \kappa t\right), D(1)+\kappa J, p=1, \ldots, 4\right\rangle$, where $\left(\theta^{p 1}(t), \theta^{p 2}(t)\right)$ are linearly independent solutions of the system $\theta_{t t}^{1}-2 \kappa \theta_{t}^{2}=\left(\kappa^{2}+\alpha\right) \theta^{1}, \theta_{t t}^{2}+2 \kappa \theta_{t}^{1}=\left(\kappa^{2}+\beta\right) \theta^{2}$.

13. $V=\frac{1}{4} h^{11}(t)|x|^{2}+i h^{00}(t): \mathfrak{g}_{V}=\left\langle M, P\left(\theta^{1}, 0\right), P\left(\theta^{2}, 0\right), P\left(0, \theta^{1}\right), P\left(0, \theta^{2}\right), J\right\rangle$, where $\left\{\theta^{1}(t), \theta^{2}(t)\right\}$ is a fundamental set of solutions of the equation $\theta_{t t}=h^{11} \theta$.

14. $V=\frac{1}{4} \alpha|x|^{2}+i \nu: \quad \mathfrak{g}_{V}=\left\langle M, P\left(\theta^{1}, 0\right), P\left(\theta^{2}, 0\right), P\left(0, \theta^{1}\right), P\left(0, \theta^{2}\right), J, D(1)\right\rangle$, where $\left\{\theta^{1}(t), \theta^{2}(t)\right\}$ is a fundamental set of solutions of the equation $\theta_{t t}=\alpha \theta$.

Remark 45. Lie invariance algebras listed in Theorem 44 are indeed maximal for the corresponding potentials if these potentials are $G^{\sim}$-inequivalent to listed potentials with larger Lie invariance algebras. Here we discuss the conditions of maximality of Lie invariance algebras for a few classification cases. Thus, in Case 3 the maximality condition is

$$
\left(\sigma_{t t}, \sigma_{t} U_{t|x|}, \sigma_{t} \operatorname{Im} U_{t t}\right) \neq(0,0,0) \quad \text { or, if } \quad \sigma_{t}=0, \quad\left(U_{|x||x|}-2|x| U_{|x|}, \operatorname{Im} U_{|x||x|}\right) \neq(0,0),
$$

which excludes the values of $V$ that are $G^{\sim}$-equivalent to those from Cases 4 and 13. Analogously, the condition $\left(\mu \neq 0\right.$ or $U_{|x||x|} \neq 2|x| U_{|x|}$ or $\left.\operatorname{Im} U_{|x||x|} \neq 0\right)$ singles out the potentials of Case 4 that are $G^{\sim}$-inequivalent to those from Case 14. The condition associated with Case 5 includes the inequality $\left(U_{t}, h_{t}^{11}\right) \neq(0,0)$ in order to exclude potentials $G^{\sim}$-equivalent to those from Case 6. To avoid the equivalence of potentials of Case 13 to those from Case 14 we require 
that $h_{t}(t) \neq 0$ or $h_{t}^{0}(t) \neq 0$ in Case 13. Analogous inequalities should be satisfied by each tuple of parameter functions appearing in listed potentials and depending only on the variable $t$. Similarly, potentials in Cases 5-9 are $G^{\sim}$-inequivalent to ones in Cases 10-14 if and only if $U_{\varpi \varpi \varpi} \neq 0$ or $\operatorname{Im} U_{\varpi} \neq 0$, where $\varpi:=x_{2}$ in Cases 5 and 6 and $\varpi:=\omega_{2}$ in Cases 8 and 9. A potential of the form given in Case 10 is $G^{\sim}$-inequivalent to a potential from Case 13 if and only if the matrix $\left(h^{a b}\right)$ is a multiple of the identity matrix, $h^{12}=h^{21}=0$ and $h^{11}=h^{22}$, i.e., the former potential itself belongs to Case 13 .

Remark 46. In Theorem 44 we neglect possible gauges of constant parameters in $V$ by discrete and scaling equivalence transformations. Thus, alternating the sign of $x_{2}$, we can set $\mu \geqslant 0$ in Case 4, and $\kappa>0$ in Cases 2, 8, 9, and 12. If $\operatorname{Im} f=0$, then we can additionally set $\nu \geqslant 0$ in Cases 11, 12 and 14 using the Wigner time reflection. Deriving the group-classification list for the class $\mathcal{V}^{\prime}$ from that for the class $\mathcal{V}^{f}$ via varying $f$, we need to regularly take into account the Wigner time reflection and the scale equivalence transformations, which correspond to linear (in $t$ ) values of the parameter function $T$. This leads to gauges $\mu \in\{0,1\}$ in Case 4 , $\alpha \in\{-1,0,1\}$ in Case 6, $\kappa=1$ in Cases 2, 8, 9, and 12, $\alpha= \pm 1$ and $\nu \geqslant 0$ in Case 11, $\alpha \in\{-1,0,1\}, \nu \geqslant 0$ and, if $\alpha=0, \nu \in\{0,1\}$ in Case 14 .

Proof. Following the discussion of Section 5.1, we single out different classification cases using the invariant integers $r_{1}, k_{2}$ and $k_{3}$. For the class $\mathcal{V}^{f}$, the general form of basis vector fields of $\mathfrak{g}_{V}$ from the complement of $\mathfrak{g}^{\cap}$ is

$$
Q^{s}=D\left(c_{s}\right)+\kappa_{s} J+P\left(\chi^{s 1}, \chi^{s 2}\right)+\sigma^{s} M,
$$

where the index $s$ runs from 1 to $\operatorname{dim} \mathfrak{g}_{V}-1, c_{s}$ and $\kappa_{s}$ are real constants, and $\chi^{s 1}, \chi^{s 2}$ and $\sigma^{s}$ are real-valued functions of $t$, and the parameter tuples $\left(c_{s}, \kappa_{s}, \chi^{s 1}, \chi^{s 2}\right)$ are linearly independent. Substituting the tuples $\left(c_{s}, \kappa_{s}, \chi^{s 1}, \chi^{s 2}, \sigma^{s}\right)$ into the classifying condition (18), we derive the system of $\operatorname{dim} \mathfrak{g}_{V}-1$ equations with respect to $V$,

$$
c_{s} V_{t}+\left(-\kappa_{s} x_{2}+\chi^{s 1}\right) V_{1}+\left(\kappa_{s} x_{1}+\chi^{s 2}\right) V_{2}=\frac{1}{2} \chi_{t t}^{s a} x_{a}+\sigma_{t}^{s} .
$$

$\boldsymbol{r}_{\mathbf{1}}=\boldsymbol{k}_{\mathbf{2}}=\mathbf{0}$. The value $k_{3}=0$ corresponds to the general Case 0 with no Lie-symmetry extension, $\mathfrak{g}_{V}=\mathfrak{g}^{\cap}$. If $k_{3}=1$, then an extension of the algebra $\mathfrak{g}_{V}$ is given by the vector field $Q^{1}=D(1)+\kappa_{1} J+P\left(\chi^{11}, \chi^{12}\right)+\sigma^{1} M$. Up to $\pi_{*} G^{\sim}$-equivalence we can set $\left(\chi^{11}, \chi^{12}\right)=(0,0)$, $\sigma^{1}=0$ and reduce $Q^{1}$ to $D(1)+\kappa_{1} J$, which results in Cases 1 and 2 depending on whether or not the parameter $\kappa$ vanishes.

$\boldsymbol{r}_{\mathbf{1}}=\mathbf{0}, \boldsymbol{k}_{\mathbf{2}}=\mathbf{1}$. The algebra $\mathfrak{g}_{V}$ contains, apart from the kernel, a vector field $Q^{0}$ with $\kappa_{0}=1$. Using $\mathcal{P}_{*}(\mathcal{X})$ with an appropriate $\mathcal{X}$, we can set $\chi^{0 a}=0$, which gives $Q^{0}=J+\sigma^{0} M$. For constant $\sigma^{0}, Q^{0}$ is reduced to $J$ by combining with $M$. Otherwise, no further reduction is possible. If $k_{3}=0$, then this extension is maximal. Integrating the equation (26) with $s=0$ yields the potential presented in Case 3 .

For $k_{3}=1$, an additional extension is provided by a vector field $Q^{1}$ with $c_{1}=1$. Combining $Q^{1}$ with $Q^{0}$ allows us to set $\kappa_{1}=0$. From the condition $\left[Q^{0}, Q^{1}\right] \in \mathfrak{g}_{V}$ we derive that $\chi^{1 a}=0$. Acting by $\mathcal{M}_{*}(\Sigma)$ with an appropriate $\Sigma$ on $\mathfrak{g}_{V}$, we also set $\sigma^{1}=0$. Finally, recalling the condition $\left[Q^{0}, Q^{1}\right] \in \mathfrak{g}_{V}$ gives $\sigma^{0}=\mu t$ with $\mu=$ const. Solving the system (26), where $s=0,1$, leads to Case 4.

$\boldsymbol{r}_{\mathbf{1}}=1$. The algebra $\mathfrak{g}_{V}$ contains a vector field $Q^{1}=P\left(\chi^{11}, \chi^{12}\right)+\sigma^{1} M$ with $\left(\chi^{11}, \chi^{12}\right) \neq(0,0)$, and $k_{2}=0$ in view of Lemma 43. The further consideration splits into two cases depending on whether or not the tuple $\left(\chi^{11}, \chi^{12}\right)$ is proportional to a constant tuple.

1. Suppose that $\chi^{11} \chi_{t}^{12}-\chi_{t}^{11} \chi^{12}=0$. Modulo $\pi_{*} G^{\sim}$-equivalence, we can set $\sigma^{1}=0, \chi^{12}=0$ and thus reduce $Q^{1}$ to $P\left(\theta^{1}, 0\right)$. Integrating the equation (26) with $s=1$, we construct the potential 
$V=U\left(t, x_{2}\right)+\frac{1}{4} h^{11}(t) x_{1}^{2}$, where $h^{11}:=\theta_{t t}^{1} / \theta^{1}$. The classifying condition (18) implies that for this value of $V$, the equation $\mathcal{L}_{V}$ admits one more Lie-symmetry vector field of the similar form, $Q^{2}=P\left(\theta^{2}, 0\right)$, where $\theta^{2}$ is a solution of the equation $\theta_{t t}=h^{11} \theta$ that is not proportional to $\theta^{1}$. If no further extensions are possible, then we have Case 5.

Otherwise, an additional extension is provided by a vector field $Q^{3}$ with $\tau^{3}=1$. The conditions $r_{1}=1$ and $\left[Q^{1}, Q^{3}\right] \in \mathfrak{g}_{V}$ require $\kappa_{3}=0$. Successively acting on $\mathfrak{g}_{V}$ by $\mathcal{P}_{*}\left(0, \mathcal{X}^{2}\right)$ and $\mathcal{M}_{*}(\Sigma)$ with appropriately chosen values of the parameter functions $\mathcal{X}^{2}$ and $\Sigma$, we can set $\chi^{32}=0$ and $\sigma^{3}=0$. Further, the condition $\left[Q^{1}, Q^{3}\right]=P\left(\theta_{t}^{1}, 0\right)+\frac{1}{2}\left(\chi^{31} \theta_{t}^{1}-\chi_{t}^{31} \theta^{1}\right) M \in$ $\left\langle Q^{1}, Q^{2}, M\right\rangle$ implies that $\chi^{31} \theta_{t}^{1}-\chi_{t}^{31} \theta^{1}=$ const, i.e., $\chi^{31} \theta_{t t}^{1}-\chi_{t t}^{31} \theta^{1}=0$ and thus $\chi_{t t}^{31}=h^{11} \chi^{31}$. This means that $\chi^{31} \in\left\langle\theta^{1}, \theta^{2}\right\rangle$. Therefore this parameter function can be set to zero by linear combining $Q^{3}$ with $Q^{1}$ and $Q^{2}$, which reduces $Q^{3}$ to $D(1)$ and gives Case 6 , where $V_{t}=0$, i.e., $h_{t}^{11}=0$ and $U_{t}=0$.

2. Let $\chi^{11} \chi_{t}^{12}-\chi_{t}^{11} \chi^{12} \neq 0$.

If $k_{3}=0$, then the vector field $Q^{1}$ reduces to the form $Q^{1}=P\left(\chi^{11}, \chi^{12}\right)$ up to $\pi_{*} G^{\sim}$ equivalence. The equation (26) with $s=1$ integrates to the expression for $V$ presented in Case 7 .

For $k_{3}=1$, there is an additional symmetry extension provided by a vector field $Q^{2}$ with $c_{2}=1$. We reduce $Q^{2}$ to $D(1)+\kappa_{2} J$. Since the commutator $\left[Q^{1}, Q^{2}\right]$ belongs to $\mathfrak{g}_{V}$, we have the equations

$$
\chi_{t}^{11}+\kappa_{2} \chi^{12}=\alpha \chi^{11}, \quad \chi_{t}^{12}-\kappa_{2} \chi^{11}=\alpha \chi^{12}, \quad \sigma_{t}^{1}=\alpha \sigma^{1}+\beta,
$$

where $\alpha$ and $\beta$ are real constants. In view of (27), for $\kappa_{2}=0$ the functions $\chi^{11}$ and $\chi^{12}$ are necessarily proportional. Consequently, $\kappa_{2}=: \kappa \neq 0$.

For $\alpha \neq 0$, modulo shifts of $t$ and combining with $M$, we have $Q^{1}=P\left(e^{\alpha t} \cos \kappa t, e^{\alpha t} \sin \kappa t\right)+$ $\beta e^{\alpha t} M$ with a real constant $\beta$. Push-forwarding vector fields from $\mathfrak{g}_{V}$ by $\mathcal{P}_{*}(-\beta \sin \kappa t, \beta \cos \kappa t)$, we set $\beta=0$. The general solution of the corresponding equations (26) with $s=1,2$ is presented in Case 8.

Let $\alpha=0$. Solving the system (27), we obtain the vector field $Q^{1}$, which takes, after linearly combining with $M$, the form $Q^{1}=P(\cos \kappa t, \sin \kappa t)+\mu t M$. No further simplification of $Q^{1}$ preserving $Q^{2}$ is possible. Thus, we obtain Case 9 .

$\boldsymbol{r}_{\mathbf{1}}=\mathbf{2}$. The algebra $\mathfrak{g}_{V}$ contains two vector fields of the form $Q^{a}=P\left(\chi^{a 1}, \chi^{a 2}\right)+\sigma^{a} M$, where $a=$ 1,2 and $\chi^{11} \chi^{22}-\chi^{12} \chi^{21} \neq 0$. The equations (26) with $s=1,2$ imply that $V_{a}=\frac{1}{2} h^{a b}(t) x_{b}+h^{0 a}(t)$, where the coefficients $h^{a b}, h^{0 a}$ are real-valued functions of $t$. Since $V_{12}=V_{21}$, the matrix $\left(h^{a b}\right)$ is symmetric and hence the potential $V$ is a quadratic polynomial in $x_{1}$ and $x_{2}$ with the coefficients being functions of $t$, and only the coefficient of the zeroth-degree summand may have a nonzero imaginary part,

$$
V=\frac{1}{4} h^{a b}(t) x_{a} x_{b}+h^{0 b}(t) x_{b}+\tilde{h}^{00}(t)+i h^{00}(t) .
$$

The subclass $\mathcal{V}_{\mathrm{q}}^{f}$ of the class $\mathcal{V}^{f}$ with potentials of the form (28) is normalized. The coefficients $h^{0 b}$ and $\tilde{h}^{00}$ can be set equal to zero up to $\pi_{*} G^{\sim}$-equivalence, which reduces the potential $V$ to the form

$$
V=\frac{1}{4} h^{a b}(t) x_{a} x_{b}+i h^{00}(t)
$$

The equations (26) with this $V$ are split with respect to different powers of $\left(x_{1}, x_{2}\right)$, yielding the systems

$$
\begin{aligned}
& c_{s} h_{t}^{11}+2 \kappa_{s} h^{12}=0, \quad c_{s} h_{t}^{12}+\kappa_{s}\left(h^{22}-h^{11}\right)=0, \quad c_{s} h_{t}^{22}-2 \kappa_{s} h^{12}=0, \quad c_{s} h_{t}^{00}=0, \\
& \chi_{t t}^{s a}=h^{a b} \chi^{s b}, \quad \sigma_{t}^{s}=0 .
\end{aligned}
$$


In view of the system (31), the algebra $\mathfrak{g}_{V}$ in fact contains, in addition to $M$, four vector fields $Q^{p}=P\left(\chi^{p 1}, \chi^{p 2}\right)$, where the tuples $\left(\chi^{p 1}, \chi^{p 2}\right), p=1, \ldots, 4$, constitute a fundamental set of solutions of the system $\chi_{t t}^{a}=h^{a b} \chi^{b}$. Moreover, we can set $\chi^{s 1}=\chi^{s 2}=\sigma^{s}=0$ for $s \neq 1, \ldots, 4$ by linearly combining $Q^{s}$ with $Q^{1}, \ldots, Q^{4}$ and $M$. Note that due to (30), the condition $k_{2}=1$ is equivalent to having $h^{11}=h^{22}$ and $h^{12}=0$.

We analyze different cases depending on values of $k_{2}$ and $k_{3}$.

1. $k_{2}=k_{3}=0$. This is the general case within the subclass $\mathcal{V}_{\mathrm{q}}^{f}$ with no further Lie symmetry extensions, which is represented by Case 10 .

2. $k_{2}=0, k_{3}=1$. The additional Lie symmetry extension is provided by the vector field $Q^{5}=$ $D(1)+\kappa_{5} J$.

If $\kappa_{5}=0$, the system (30) with $s=5$ is equivalent to that all $h^{a b}$ and $h^{0 a}$ are constants. Up to rotations, we can reduce the matrix $\left(h^{a b}\right)$ to a diagonal matrix $\operatorname{diag}(\alpha, \beta)$ with $\beta \neq \alpha \neq 0$, obtaining Case 11.

Otherwise, up to translations of time, the general solution of the system (30) with $s=5$ is $h^{11}=\alpha \cos ^{2} t+\beta \sin ^{2} t, h^{12}=h^{21}=(\alpha-\beta) \cos t \sin t, h^{22}=\alpha \sin ^{2} t+\beta \cos ^{2} t, h^{00}=\nu$, where $\alpha, \beta$ and $\nu$ are arbitrary real constants with $\alpha \neq \beta$ due to the auxiliary conditions for the subclass $\mathcal{V}_{0}^{f}$. We integrate the system (31) with the above values of $h^{a b}$, rearranging the potential $V$ in terms of $\omega_{a}$. This provides Case 12 .

3. $k_{2}=1, k_{3}=0$. The additional Lie-symmetry extension is provided by the vector field $Q^{0}=J$, which corresponds to Case 13.

4. $k_{2}=1, k_{3}=1$. We obviously obtain Case 14 , where there is one more Lie-symmetry vector field $Q^{5}=D(1)$ in comparison to Case 13 .

\subsection{Logarithmic modular nonlinearity}

We recall that the class of Schrödinger equations with a logarithmic modular nonlinearity with a fixed $\delta$ is denoted by $\mathcal{P}_{0}^{\delta}$ and consists of equations of the form (19). For any equation $\mathcal{L}_{V}$ from the class $\mathcal{P}_{0}^{\delta}$ with $n=2$, the results from Section 5.2 imply that

$$
\operatorname{dim} \mathfrak{g}_{V} \leqslant 8, \quad r_{1} \in\{0,1,2\}, \quad k_{0}=2, \quad k_{1} \in\{0, \ldots, 4\}, \quad k_{2} \in\{0,1\}, \quad k_{3} \in\{0,1\} .
$$

It then follows that any appropriate subalgebra of $\mathfrak{g}_{\langle>}$is spanned by

- the basis vector fields $M$ and $I$ of the kernel $\mathfrak{g}^{\cap}$,

- $k_{1}$ vector fields $P\left(\chi^{p 1}, \chi^{p 2}\right)+\sigma^{p} M+\zeta^{p} I$ with linearly independent tuples $\left(\chi^{p 1}, \chi^{p 2}\right), p=$ $1, \ldots, k_{1}$,

- $k_{2}$ vector fields $J+P\left(\chi^{01}, \chi^{02}\right)+\sigma^{0} M+\zeta^{0} I$,

- $k_{3}$ vector fields $D(1)+\kappa_{q} J+P\left(\chi^{q 1}, \chi^{q 2}\right)+\sigma^{q} M+\zeta^{q} I$ with $q=k_{1}+k_{3}$.

We will also use the following notation:

$$
\begin{aligned}
& I^{\prime}=e^{-\delta_{2} t}\left(\delta_{2} I-\delta_{1} M\right) \quad \text { if } \quad \delta_{2} \neq 0 \quad \text { and } \quad I^{\prime}=I+\delta_{1} t M \quad \text { if } \quad \delta_{2}=0, \\
& P^{\prime}\left(\chi^{1}, \chi^{2}\right)=P\left(\chi^{1}, \chi^{2}\right)-\hat{\zeta} I-\delta_{1} \int \hat{\zeta} \mathrm{d} t M \quad \text { with } \hat{\zeta}=e^{-\delta_{2} t} \int e^{\delta_{2} t} h^{0 b} \chi^{b} \mathrm{~d} t,
\end{aligned}
$$

where all involved parameters will be explained in the corresponding places, $\delta_{1}:=\operatorname{Re} \delta$ and $\delta_{2}:=\operatorname{Im} \delta$, and we assume that $h^{0 a}$ is the imaginary part of the coefficient of the summand of the first degree in $x_{a}$ if this summand is explicitly presented in the corresponding potential $V$, otherwise $h^{0 a}:=0$. For example, $h^{01}=\nu^{1} \cos \kappa t-\nu_{2} \sin \kappa t$ and $h^{02}=\nu^{1} \sin \kappa t+\nu_{2} \cos \kappa t$ in Case 12 below. 
Theorem 47. A complete list of inequivalent Lie symmetry extensions in the class $\mathcal{P}_{0}^{\delta}$ is exhausted by the cases listed below, where $U$ is an arbitrary complex-valued smooth function of its arguments or an arbitrary complex constant, and the other functions and constants take real values.

0. $V=V(t, x): \quad \mathfrak{g}_{V}=\mathfrak{g}^{\bigcap}=\left\langle M, I^{\prime}\right\rangle$.

1. $V=U\left(x_{1}, x_{2}\right): \mathfrak{g}_{V}=\left\langle M, I^{\prime}, D(1)\right\rangle$.

2. $V=U\left(\omega_{1}, \omega_{2}\right): \mathfrak{g}_{V}=\left\langle M, I^{\prime}, D(1)+\kappa J, \kappa \neq 0\right\rangle$.

3. $V=U(t,|x|)+\left(\sigma_{t}-i \zeta_{t}-\delta \zeta\right) \phi: \mathfrak{g}_{V}=\left\langle M, I^{\prime}, J+\sigma(t) M+\zeta(t) I\right\rangle$.

4. $V=U(|x|)+\left(\mu-\delta^{\prime} \nu\right) \phi$,

$\delta_{2} \neq 0: \quad \delta^{\prime}:=\delta_{1}+i \delta_{2}, \quad \mathfrak{g}_{V}=\left\langle M, I^{\prime}, J+\mu t M+\nu I, D(1)\right\rangle$,

$\delta_{2}=0: \quad \delta^{\prime}:=i, \quad \mathfrak{g}_{V}=\left\langle M, I^{\prime}, J+\left(\mu t+\frac{1}{2} \nu \delta_{1} t^{2}\right) M+\nu t I, D(1)\right\rangle$.

5. $V=U\left(t, x_{2}\right)+\frac{1}{4} h^{11}(t) x_{1}^{2}+i h^{01}(t) x_{1}: \mathfrak{g}_{V}=\left\langle M, I^{\prime}, P^{\prime}\left(\theta^{1}, 0\right), P^{\prime}\left(\theta^{2}, 0\right)\right\rangle$,

where $\theta^{1}$ and $\theta^{2}$ are linearly independent solutions of the equation $\theta_{t t}=h^{11} \theta$.

6. $V=U\left(x_{2}\right)+\frac{1}{4} \alpha x_{1}^{2}+i \nu x_{1}: \mathfrak{g}_{V}=\left\langle M, I^{\prime}, P^{\prime}\left(\theta^{1}, 0\right), P^{\prime}\left(\theta^{2}, 0\right), D(1)\right\rangle$, where $\theta^{1}$ and $\theta^{2}$ are linearly independent solutions of the equation $\theta_{t t}=\alpha \theta$.

7. $V=U(t, \varpi)+\frac{\chi_{t t}^{11}}{4 \chi^{11}} x_{1}^{2}+\frac{\chi_{t t}^{12}}{4 \chi^{12}} x_{2}^{2}+i h^{01}(t) x_{1}, \varpi=\chi^{11} x_{2}-\chi^{12} x_{1}, \quad \chi_{t}^{11} \chi^{12} \neq \chi^{11} \chi_{t}^{12}$ : $\mathfrak{g}_{V}=\left\langle M, I^{\prime}, P^{\prime}\left(\chi^{11}, \chi^{12}\right)\right\rangle$.

8. $V=U\left(\omega_{2}\right)+\frac{1}{4}\left(\alpha^{2}-\kappa^{2}\right) \omega_{1}^{2}+\alpha \kappa \omega_{1} \omega_{2}+i \nu \omega_{1}, \quad \alpha \kappa \neq 0$ : $\mathfrak{g}_{V}=\left\langle M, I^{\prime}, P^{\prime}\left(e^{\alpha t} \cos \kappa t, e^{\alpha t} \sin \kappa t\right), D(1)+\kappa J\right\rangle$.

9. $V=U\left(\omega_{2}\right)-\frac{1}{4} \kappa^{2} \omega_{1}^{2}+(\mu+i \nu) \omega_{1}, \kappa \neq 0: \quad \mathfrak{g}_{V}=\left\langle M, I^{\prime}, P^{\prime}(\cos \kappa t, \sin \kappa t)+\mu t M, D(1)+\kappa J\right\rangle$.

10. $V=\frac{1}{4} h^{a b}(t) x_{a} x_{b}+i h^{0 b}(t) x_{b}, h^{12}=h^{21}: \mathfrak{g}_{V}=\left\langle M, I^{\prime}, P^{\prime}\left(\chi^{p 1}, \chi^{p 2}\right), p=1, \ldots, 4\right\rangle$, where $\left\{\left(\chi^{p 1}(t), \chi^{p 2}(t)\right)\right\}$ is a fundamental set of solutions of the system $\chi_{t t}^{a}=h^{a b} \chi^{b}$.

11. $V=\frac{1}{4} \alpha x_{1}^{2}+\frac{1}{4} \beta x_{2}^{2}+i \nu_{a} x_{a}, \beta \neq \alpha \neq 0$ : $\mathfrak{g}_{V}=\left\langle M, I^{\prime}, P^{\prime}\left(\theta^{1}, 0\right), P^{\prime}\left(\theta^{2}, 0\right), P^{\prime}\left(0, \theta^{3}\right), P^{\prime}\left(0, \theta^{4}\right), D(1)\right\rangle$, where $\left\{\theta^{1}(t), \theta^{2}(t)\right\}$ and $\left\{\theta^{3}(t), \theta^{4}(t)\right\}$ are fundamental sets of solutions of the equations $\theta_{t t}=\alpha \theta$ and $\theta_{t t}=\beta \theta$, respectively.

12. $V=\frac{1}{4} \alpha \omega_{1}^{2}+\frac{1}{4} \beta \omega_{2}^{2}+i \nu_{a} \omega_{a}, \beta \neq \alpha \neq 0, \quad \kappa \neq 0$ : $\mathfrak{g}_{V}=\left\langle M, I^{\prime}, P^{\prime}\left(\theta^{p 1} \cos \kappa t-\theta^{p 2} \sin \kappa t, \theta^{p 1} \sin \kappa t+\theta^{p 2} \cos \kappa t\right), D(1)+\kappa J, p=1, \ldots, 4\right\rangle$, where $\left(\theta^{p 1}(t), \theta^{p 2}(t)\right)$ are linearly independent solutions of the system $\theta_{t t}^{1}-2 \kappa \theta_{t}^{2}=\left(\kappa^{2}+\alpha\right) \theta^{1}, \theta_{t t}^{2}+2 \kappa \theta_{t}^{1}=\left(\kappa^{2}+\beta\right) \theta^{2}$.

13. $V=\frac{1}{4} h(t)|x|^{2}: \quad \mathfrak{g}_{V}=\left\langle M, I^{\prime}, P\left(\chi^{p 1}, \chi^{p 2}\right), p=1, \ldots, 4, J\right\rangle$, where $\left\{\left(\chi^{p 1}(t), \chi^{p 2}(t)\right)\right\}$ is a fundamental set of solutions of the system $\chi_{t t}=h \chi$.

14. $V=\frac{1}{4} \alpha|x|^{2}: \quad \mathfrak{g}_{V}=\left\langle M, I^{\prime}, P\left(\chi^{1}, 0\right), P\left(\chi^{2}, 0\right), P\left(0, \chi^{1}\right), P\left(0, \chi^{2}\right), J, D(1)\right\rangle$, where $\left(\chi^{1}(t), \chi^{2}(t)\right)$ is a fundamental set of solutions of the equation $\chi_{t t}=\alpha \chi$.

Remark 48. The conditions of maximality of Lie invariance algebras presented in Theorem 47 are similar to those discussed in Remark 45 for algebras listed in Theorem 44. The required modifications are obvious. For example, in Case 3 the maximality condition is $\left(\sigma_{t t}-i \zeta_{t t}-\right.$ $\left.\delta \zeta_{t},\left(\sigma_{t}-i \zeta_{t}-\delta \zeta\right) U_{t|x|}\right) \neq(0,0)$ or, if $\sigma_{t}-i \zeta_{t}-\delta \zeta=0$, it is $\left(U_{|x||x|}-2|x| U_{|x|}, \operatorname{Im} U_{|x||x|}\right) \neq(0,0)$, which excludes the values of $V$ that is $G^{\sim}$-equivalent to those from Cases 4 and 13. A potential of the form presented in Case 10 is $G^{\sim}$-equivalent to a potential from Case 13 if and only if the matrix $\left(h^{a b}\right)$ is a multiple of the identity matrix, $h^{12}=h^{21}=0$ and $h^{11}=h^{22}$, and $h^{0 a}=0$, i.e., the former potential itself belongs to Case 13 . 
Remark 49. In Theorem 47, we also neglect some possible gauges of constant parameters in $V$. Thus, alternating the signs of $x_{2}$ and/or $x_{1}$, we can set $\mu \geqslant 0$ and, if $\mu=0$, we can make $\nu \geqslant 0$ in Cases 4 and 9 and $\kappa>0$ in Cases 2, 8, 9 and 12. If $\operatorname{Im} \delta=0$, then using the Wigner time reflection we can additionally set $\nu \geqslant 0$ in Cases 4,6,8 and 9 and make one of nonzero $\nu_{a}$ positive in Cases 11, 12 and 14. In contrast to the class $\mathcal{V}^{\prime}$, in the course of group classification of the entire class $\mathcal{P}_{0}$, we have no additional possibilities for gauging constant parameters in $V$.

Proof. Similar to the proof of Theorem 44, we follow the discussion of Section 5.2 and single out different classification cases using the invariant integers $r_{1}, k_{2}$ and $k_{3}$. At the same time, this proof essentially differs in some points from the proof of Theorem 44 due to the appearance of the vector fields $\zeta I$ in $\mathfrak{g}_{\langle\rangle}$and the extension of the kernel Lie invariance algebra with $I^{\prime}$. For the class $\mathcal{P}_{0}^{\delta}$, the general form of basis vector fields of $\mathfrak{g}_{V}$ from the complement of $\mathfrak{g}^{\cap}$ is

$$
Q^{s}=D\left(c_{s}\right)+\kappa_{s} J+P\left(\chi^{s 1}, \chi^{s 2}\right)+\sigma^{s} M+\zeta^{s} I,
$$

where the range of $s$ is equal to $\operatorname{dim} \mathfrak{g}_{V}-2, c_{s}$ and $\kappa_{s}$ are real constants, $\chi^{s 1}, \chi^{s 2}, \sigma^{s}$ and $\zeta^{s}$ are real-valued functions of $t$, and the parameter tuples $\left(c_{s}, \kappa_{s}, \chi^{s 1}, \chi^{s 2}\right)$ are linearly independent. Substituting the tuples $\left(c_{s}, \kappa_{s}, \chi^{s 1}, \chi^{s 2}, \sigma^{s}, \zeta^{s}\right)$ into the classifying condition (21), we derive the system of $\operatorname{dim} \mathfrak{g}_{V}-2$ equations with respect to $V$,

$$
c_{s} V_{t}+\left(-\kappa_{s} x_{2}+\chi^{s 1}\right) V_{1}+\left(\kappa_{s} x_{1}+\chi^{s 2}\right) V_{2}=\frac{1}{2} \chi_{t t}^{s a} x_{a}+\sigma_{t}^{s}-\delta_{1} \zeta^{s}-i\left(\zeta_{t}^{s}+\delta_{2} \zeta^{s}\right) .
$$

We operate with the parameters $c_{s}, \kappa_{s}, \chi^{s 1}, \chi^{s 2}$ and $\sigma^{s}$ within the class $\mathcal{P}_{0}^{\delta}$ in the same way as we did within the class $\mathcal{V}^{f}$. This is why below we assume all the reductions for these parameters having been carried out and present only the points where the presence of $\zeta I$ in $\mathfrak{g}_{\langle>}$crucially modifies the proof. Each case of this theorem corresponds to the case of Theorem 44 with the same number. The derivation of Cases 0-3 is essentially the same as in the proof of Theorem 44 .

$r_{1}=0, k_{2}=1, k_{3}=1$. Up to $\pi_{*} G^{\sim}$-equivalence, the Lie-symmetry extension can be assumed to be provided by $Q^{0}=J+\sigma M+\zeta I$ and $Q^{1}=D(1)$. The condition $\left[Q^{1}, Q^{0}\right] \in \mathfrak{g}_{V}$ reduces to $\left[Q^{1}, Q^{0}\right]=\sigma_{t} M+\zeta_{t} I \in\left\langle M, I^{\prime}\right\rangle$. This gives expressions for the derivatives $\sigma_{t}$ and $\zeta_{t}$, which depend on whether or not $\delta_{2}$ vanishes. Integrating these expressions up to $\pi_{*} G^{\sim}$-equivalence, we find $\sigma=\mu t, \zeta=\nu$ if $\delta_{2} \neq 0$ and $\sigma=\mu t+\frac{1}{2} \nu \delta_{1} t^{2}, \zeta=\nu t$ if $\delta_{2}=0$. The equations (32) with $s=0,1$ leads to the expression for $V$ in Case 4 .

$r_{1}=1$. Then $k_{2}=0$, and, the algebra $\mathfrak{g}_{V}$ contains, in addition to $M$, at least a vector field $Q^{1}=$ $P\left(\chi^{11}, \chi^{12}\right)+\sigma^{1} M+\zeta^{1} I$ with $\left(\chi^{11}, \chi^{12}\right) \neq(0,0)$. The further analysis depends on whether or not the parameter functions $\chi^{11}$ and $\chi^{12}$ are linearly dependent.

1. $\chi_{t}^{11} \chi^{12}=\chi^{11} \chi_{t}^{12}$. Up to $G^{\sim}$-equivalence, we can set $\chi^{12}=0$. It is then convenient to re-denote $\theta^{1}:=\chi^{11}$. Then integrating the equation (32) with $s=1$ with respect to $V$ gives

$$
V=U\left(t, x_{2}\right)+\frac{1}{4} h^{11}(t) x_{1}^{2}+i h^{01}(t) x_{1}+\tilde{h}^{01}(t) x_{1},
$$

where $h^{11}, h^{01}$ and $\tilde{h}^{01}$ are smooth real-valued functions of $t$.

If $k_{3}=0$, then we make the coefficient $\tilde{h}^{01}$ zero using equivalence transformations. For this potential, the classifying condition (21) implies that the functions involved in $Q^{1}$ satisfy the system $\theta_{t t}^{1}=h^{11} \theta^{1}, \zeta_{t}^{1}+\delta_{2} \zeta^{1}=-h^{01} \chi^{11}$ and $\sigma_{t}^{1}=\delta_{1} \zeta^{1}$, i.e., $Q^{1}=P^{\prime}\left(\theta^{1}, 0\right)$. Moreover, the algebra $\mathfrak{g}_{V}$ contains one more vector field of the similar form, $Q^{2}=P^{\prime}\left(\theta^{2}, 0\right)$, where $\theta^{2}$ is a solution of the equation $\theta_{t t}=h^{11} \theta$ that is linearly independent with $\theta^{1}$. As a result, we have Case 5.

For $k_{3}=1$, an additional Lie-symmetry extension is provided by a vector field $Q^{3}$ with $\tau^{3}=1$. The conditions $r_{1}=1$ and $\left[Q^{1}, Q^{3}\right] \in \mathfrak{g}_{V}$ require $\kappa_{3}=0$. Successively acting on $\mathfrak{g}_{V}$ 
by $\mathcal{P}_{*}(\mathcal{X}), \mathcal{M}_{*}(\Sigma)$ and $\mathcal{I}_{*}(Z)$ with appropriately chosen values of the parameter functions $\mathcal{X}^{a}$, $\Sigma$ and $Z$, we can set $\chi^{3 a}=0, \sigma^{3}=0$ and $\zeta^{3}=0$. Then the equation (32) with $s=3$ takes the form $V_{t}=0$ and implies that the coefficients $h^{11}, h^{01}$ and $\tilde{h}^{01}$ in the representation (33) for $V$ are constants. Acting by $\mathcal{P}\left(\tilde{h}^{01}, 0\right)$ on $\mathcal{L}_{V}$, we annihilate $\tilde{h}^{01}$. Repeating the argumentation from the case $k_{3}=0$ leads to Case 6 .

2. $\chi_{t}^{11} \chi^{12} \neq \chi^{11} \chi_{t}^{12}$. The equation (32) with $s=1$ integrates with respect to $V$ to

$$
V=U(t, \varpi)+\frac{\chi_{t t}^{11}}{4 \chi^{11}} x_{1}^{2}+\frac{\chi_{t t}^{12}}{4 \chi^{12}} x_{2}^{2}+i h^{01}(t) x_{1}+\tilde{h}^{01}(t) x_{1}
$$

where $\varpi:=\chi^{11} x_{2}-\chi^{12} x_{1}$, and $h^{01}$ and $\tilde{h}^{01}$ are smooth real-valued functions of $t$.

If $k_{3}=0$, then we make the coefficient $\tilde{h}^{01}$ zero using equivalence transformations. For this potential, the classifying condition (21) implies that the functions involved in $Q^{1}$ satisfy the system $\zeta_{t}^{1}+\delta_{2} \zeta^{1}=-h^{01} \chi^{11}$ and $\sigma_{t}^{1}=\delta_{1} \zeta^{1}$, i.e., $Q^{1}=P^{\prime}\left(\chi^{11}, \chi^{12}\right)$, which corresponds to Case 7 .

If $k_{3}=1$, then modulo $\pi_{*} G^{\sim}$-equivalence we reduce a vector field $Q^{2}$ with $\tau^{2}=1$, which provides an additional Lie-symmetry extension, to the form $Q^{2}=D(1)+\kappa_{2} J$. In view of the condition $\left[Q^{2}, Q^{1}\right] \in \mathfrak{g}_{V}$ and modulo shifts of $t$ and combining with $M$, we have $\left(\chi^{11}, \chi^{12}\right)=$ $\left(e^{\alpha t} \cos \kappa_{2} t, e^{\alpha t} \sin \kappa_{2} t\right)$. The equations (32) with $s=1,2$ simultaneously integrate with respect to $V$ to

$$
V=U\left(\omega_{2}\right)+\frac{1}{4}\left(\alpha^{2}-\kappa_{2}^{2}\right) \omega_{1}^{2}+\alpha \kappa_{2} \omega_{1} \omega_{2}+(\mu+i \nu) \omega_{1} .
$$

Here $\alpha, \mu$ and $\nu$ are real constants such that $\zeta_{t}^{1}+\delta_{2} \zeta^{1}=-\nu$ and $\sigma_{t}^{1}=\delta_{1} \zeta^{1}+\mu$. If $\alpha \neq 0$, then acting by $\mathcal{P}(-\mu \cos t, \mu \sin t)$ on $\mathcal{L}_{V}$, we set $\mu=0$. For $\alpha=0$, annihilating $\mu$ if it is nonzero is impossible. After integrating the above system for $\left(\sigma^{1}, \zeta^{1}\right)$ and linearly combining $Q^{1}$ with $M$ and $I^{\prime}$, we obtain Cases 8 and 9 for $\alpha \neq 0$ and $\alpha=0$, respectively.

$\boldsymbol{r}_{\mathbf{1}}=\mathbf{2}$. Similarly to the case $r_{1}=2$ in the proof of Theorem 44 , here the potential $V$ is quadratic in $x$,

$$
V=\frac{1}{4} h^{a b}(t) x_{a} x_{b}+\tilde{h}^{0 b}(t) x_{b}+i h^{0 b}(t) x_{b}+h^{00}(t)+i \tilde{h}^{00}(t),
$$

where the functions $\tilde{h}^{0 b}, h^{00}, \tilde{h}^{00}$ can be set to zero up to $G^{\sim}$-equivalence. So, it suffices to study potentials of the form

$$
V=\frac{1}{4} h^{a b}(t) x_{a} x_{b}+i h^{0 b}(t) x_{b} .
$$

Splitting the equations (32) in view of (35), with respect to different powers of $\left(x_{1}, x_{2}\right)$ leads to the systems

$$
\begin{aligned}
& c_{s} h_{t}^{11}+2 \kappa_{s} h^{12}=0, \quad c_{s} h_{t}^{12}+\kappa_{s}\left(h^{22}-h^{11}\right)=0, \quad c_{s} h_{t}^{22}-2 \kappa_{s} h^{12}=0, \\
& c_{s} h_{t}^{01}+\kappa_{s} h^{02}=0, \quad c_{s} h_{t}^{02}-\kappa_{s} h^{01}=0, \\
& \chi_{t t}^{s a}=h^{a b} \chi^{s b}, \quad \sigma_{t}^{s}=\delta_{1} \zeta^{s}, \quad \zeta_{t}^{s}+\delta_{2} \zeta^{s}=-h^{0 b} \chi^{s b} .
\end{aligned}
$$

In view of the system (38), the algebra $\mathfrak{g}_{V}$ necessarily contains, in additional to $M$ and $I^{\prime}$, four vector fields $Q^{p}=P^{\prime}\left(\chi^{p 1}, \chi^{p 2}\right)$, where the tuples $\left(\chi^{p 1}, \chi^{p 2}\right), p=1, \ldots, 4$, constitute a fundamental set of solutions of the system $\chi_{t t}^{a}=h^{a b} \chi^{b}$. Moreover, we can set $\chi^{s 1}=\chi^{s 2}=\sigma^{s}=$ $\zeta^{s}=0, s \neq 1, \ldots, 4$, by linearly combining $Q^{s}$ with $Q^{1}, \ldots, Q^{4}, M$ and $I^{\prime}$. Further analysis, which depends on values of $k_{2}$ and $k_{3}$, is analogous to the proof of Theorem 44 and results in Cases 10-14. Note only that due to (36) and (37), here the condition $k_{2}=1$ is equivalent to having $h^{11}=h^{22}$ and $h^{12}=h^{0 b}=0$. 


\subsection{Power modular nonlinearity}

We solve the group classification problem of the class $\mathcal{P}_{\lambda}^{\delta}$ of nonlinear Schrödinger equations with potentials and power nonlinearity with a fixed $\delta, \delta \in \mathbb{C}_{0}$ for $n=2$. For any potential $V$ in an equation of this class we have

$\operatorname{dim} \mathfrak{g}_{V} \leqslant 9, \quad r_{1} \in\{0,1,2\}, \quad k_{0}=1, \quad k_{1} \in\{0, \ldots, 4\}, \quad k_{2} \in\{0,1\}, \quad k_{3} \in\{0,1,2,3\}$,

and $\lambda^{\prime}=\frac{1}{\lambda}-\frac{1}{2}$. The algebra $\mathfrak{g}_{V}$ is spanned by the following vector fields:

- the basis vector field $M$ of the kernel $\mathfrak{g}^{\cap}$,

- $k_{1}$ vector fields $P\left(\chi^{p 1}, \chi^{p 2}\right)+\sigma^{p} M$ with linearly independent tuples $\left(\chi^{p 1}, \chi^{p 2}\right)$ and $p=$ $1, \ldots, k_{1}$,

- $k_{2}$ vector fields of the form $J+P\left(\chi^{01}, \chi^{02}\right)+\sigma^{0} M$,

- $k_{3}$ vector fields $D^{\lambda}\left(\tau^{q}\right)+\kappa_{q} J+P\left(\chi^{q 1}, \chi^{q 2}\right)+\sigma^{q} M, q=k_{1}+1, \ldots, k_{1}+k_{3}$ with linearly independent $\tau^{k_{1}+1}, \ldots, \tau^{k_{1}+k_{3}}$.

Theorem 50. A complete list of inequivalent Lie symmetry extensions in the class $\mathcal{P}_{\lambda}^{\delta}$ is exhausted by the cases listed below, where $U$ is an arbitrary complex-valued smooth function of its arguments or an arbitrary complex constant, and the other functions and constants take real values.

0. $V=V(t, x): \quad \mathfrak{g}_{V}=\mathfrak{g}^{\cap}=\langle M\rangle$.

1. $V=U\left(x_{1}, x_{2}\right): \mathfrak{g}_{V}=\langle M, D(1)\rangle$.

2. $V=U\left(\omega_{1}, \omega_{2}\right), \quad \kappa \neq 0: \quad \mathfrak{g}_{V}=\langle M, D(1)+\kappa J\rangle$.

3. $V=|x|^{-2} U(\varpi), \varpi=\phi-2 \kappa \ln |x|, \quad \kappa \neq 0, \quad U_{\varpi} \neq 0: \quad \mathfrak{g}_{V}=\left\langle M, D(1), D^{\lambda}(t)+\kappa J\right\rangle$.

4. $V=|x|^{-2} U(\phi), \quad U_{\phi} \neq 0$,

$\lambda \neq 2: \quad \mathfrak{g}_{V}=\left\langle M, D(1), D^{\lambda}(t)\right\rangle$,

$\lambda=2: \quad \mathfrak{g}_{V}=\left\langle M, D(1), D^{\lambda}(t), D^{\lambda}\left(t^{2}\right)\right\rangle$.

5. $V=U(t,|x|)+\mu \phi: \quad \mathfrak{g}_{V}=\langle M, J+\mu t M\rangle$.

6. $V=U(|x|)+\mu \phi: \quad \mathfrak{g}_{V}=\langle M, J+\mu t M, D(1)\rangle$.

7. $V=|x|^{-2} U, \quad U \neq 0$,

$\lambda \neq 2: \quad \mathfrak{g}_{V}=\left\langle M, J, D(1), D^{\lambda}(t)\right\rangle$,

$\lambda=2: \quad \mathfrak{g}_{V}=\left\langle M, J, D(1), D^{\lambda}(t), D^{\lambda}\left(t^{2}\right)\right\rangle$.

8. $V=U\left(t, x_{2}\right): \quad \mathfrak{g}_{V}=\langle M, P(1,0), P(t, 0)\rangle$.

9. $V=U(\varpi), \varpi=x_{2}: \quad \mathfrak{g}_{V}=\langle M, P(1,0), P(t, 0), D(1)\rangle$.

10. $V=t^{-1} U(\varpi), \varpi=|t|^{-1 / 2} x_{2}: \mathfrak{g}_{V}=\left\langle M, P(1,0), P(t, 0), D^{\lambda}(t)\right\rangle$.

11. $V=\left(t^{2}+1\right)^{-1}\left(U(\varpi)+2 i \lambda^{\prime} t\right), \varpi=\left(t^{2}+1\right)^{-1 / 2} x_{2}$ :

$\mathfrak{g}_{V}=\left\langle M, P(1,0), P(t, 0), D^{\lambda}\left(t^{2}+1\right)\right\rangle$.

12. $V=x_{2}^{-2} U, U \neq 0$,

$\lambda \neq 2: \quad \mathfrak{g}_{V}=\left\langle M, P(1,0), P(t, 0), D(1), D^{\lambda}(t)\right\rangle$,

$\lambda=2: \quad \mathfrak{g}_{V}=\left\langle M, P(1,0), P(t, 0), D(1), D^{\lambda}(t), D^{\lambda}\left(t^{2}\right)\right\rangle$.

13. $V=U\left(t, \omega_{2}\right)+\frac{1}{4}\left(h_{t t}-h\right) h^{-1} \omega_{1}^{2}+h_{t} h^{-1} \omega_{1}, h=h(t) \neq 0: \quad \mathfrak{g}_{V}=\langle M, P(h \cos t, h \sin t)\rangle$. 
14. $V=U\left(\omega_{2}\right)+\frac{1}{4}\left(\alpha^{2}-\kappa^{2}\right) \omega_{1}^{2}+\alpha \kappa \omega_{1} \omega_{2}, \quad \alpha \kappa \neq 0$ : $\left.\mathfrak{g}_{V}=\left\langle M, P\left(e^{\alpha t} \cos \kappa t, e^{\alpha t} \sin \kappa t\right), D(1)+\kappa J\right\rangle\right\rangle$.

15. $V=U\left(\omega_{2}\right)-\frac{1}{4} \kappa^{2} \omega_{1}^{2}+\mu \omega_{1}, \quad \kappa \neq 0: \mathfrak{g}_{V}=\langle M, P(\cos \kappa t, \sin \kappa t)+\mu t M, D(1)+\kappa J\rangle$.

16. $V=\frac{1}{4} h^{a b}(t) x_{a} x_{b}+i h^{00}(t), h^{12}=h^{21}: \quad \mathfrak{g}_{V}=\left\langle M, P\left(\chi^{p 1}, \chi^{p 2}\right), p=1, \ldots, 4\right\rangle$, where $\left\{\left(\chi^{p 1}(t), \chi^{p 2}(t)\right)\right\}$ is a fundamental set of solutions of the system $\chi_{t t}^{a}=h^{a b} \chi^{b}$.

17. $V=\frac{1}{4} \alpha x_{1}^{2}+\frac{1}{4} \beta x_{2}^{2}+i \nu, \beta \neq \alpha \neq 0: \mathfrak{g}_{V}=\left\langle M, P\left(\theta^{1}, 0\right), P\left(\theta^{2}, 0\right), P\left(0, \theta^{3}\right), P\left(0, \theta^{4}\right), D(1)\right\rangle$, where $\left\{\theta^{1}(t), \theta^{2}(t)\right\}$ and $\left\{\theta^{3}(t), \theta^{4}(t)\right\}$ are fundamental sets of solutions of the equations $\theta_{t t}=\alpha \theta$ and $\theta_{t t}=\beta \theta$, respectively.

18. $V=\frac{1}{4} \alpha \omega_{1}^{2}+\frac{1}{4} \beta \omega_{2}^{2}+i \nu, \beta \neq \alpha \neq 0, \quad \kappa \neq 0$ :

$\mathfrak{g}_{V}=\left\langle M, P\left(\theta^{p 1} \cos \kappa t-\theta^{p 2} \sin \kappa t, \theta^{p 1} \sin \kappa t+\theta^{p 2} \cos \kappa t\right), D(1)+\kappa J, p=1, \ldots, 4\right\rangle$, where $\left(\theta^{p 1}(t), \theta^{p 2}(t)\right)$ are linearly independent solutions of the system $\theta_{t t}^{1}-2 \kappa \theta_{t}^{2}=\left(\kappa^{2}+\alpha\right) \theta^{1}, \theta_{t t}^{2}+2 \kappa \theta_{t}^{1}=\left(\kappa^{2}+\beta\right) \theta^{2}$.

19. $\left.V=i h^{00}(t): \mathfrak{g}_{V}=\langle M, P(1,0), P(t, 0), P(0,1), P(0, t), J)\right\rangle$.

20. $V=i \nu, \quad \nu \neq 0: \quad \mathfrak{g}_{V}=\langle M, P(1,0), P(t, 0), P(0,1), P(0, t), J, D(1)\rangle$.

21. $V=i \nu t^{-1}, \quad \nu \neq 0: \mathfrak{g}_{V}=\left\langle M, P(1,0), P(t, 0), P(0,1), P(0, t), J, D^{\lambda}(t)\right\rangle$.

22. $V=i\left(t^{2}+1\right)^{-1}\left(2 \lambda^{\prime} t+\nu\right), \quad\left(\lambda^{\prime}, \nu\right) \neq(0,0)$ : $\mathfrak{g}_{V}=\left\langle M, P(1,0), P(t, 0), P(0,1), P(0, t), J, D^{\lambda}\left(t^{2}+1\right)\right\rangle$.

23. $V=0$ :

$\lambda \neq 2: \quad \mathfrak{g}_{V}=\left\langle M, P(1,0), P(t, 0), P(0,1), P(0, t), J, D(1), D^{\lambda}(t)\right\rangle$,

$\lambda=2: \quad \mathfrak{g}_{V}=\left\langle M, P(1,0), P(t, 0), P(0,1), P(0, t), J, D(1), D^{\lambda}(t), D^{\lambda}\left(t^{2}\right)\right\rangle$.

Remark 51. As in Theorems 44 and 47, Lie invariance algebras listed in Theorem 50 are indeed maximal and coincide with $\mathfrak{g}_{V}$ for the corresponding potentials if these potentials are $G^{\sim}$-inequivalent to listed potentials with larger Lie invariance algebras. For Cases $3,4,12$ and 20-22, the maximality conditions are obvious and presented directly in the corresponding cases. A potential of the form given in Case 16 is $G^{\sim}$-equivalent to a potential from Case 19 if and only if $h^{12}=h^{21}=0$ and $h^{11}=h^{22}$, i.e., the former potential itself belongs to Case 19. At the same time, the maximality conditions for a number of cases of Lie-symmetry extensions within the class $\mathcal{P}_{\lambda}^{\delta}$ are more cumbersome than similar conditions within the classes $\mathcal{V}^{f}$ and $\mathcal{P}_{0}^{\delta}$. This is caused by the fact that the class $\mathcal{P}_{\lambda}^{\delta}$ admits equivalence transformations with nonconstant $T_{t}$, which complicates describing $G^{\sim}$-equivalent cases. Moreover, there are a number of cases of Lie symmetry extensions within the class $\mathcal{P}_{\lambda}^{\delta}$ that have no counterparts among Lie symmetry extensions within the classes $\mathcal{V}^{f}$ and $\mathcal{P}_{0}^{\delta}$ since they are associated with the values of $k_{3}$ in $\{2,3\}$. For example, the maximality condition for Case 5,

$$
\mu\left(U_{t|x|}, \operatorname{Im} U_{t t}\right) \neq(0,0) \quad \text { or, if } \mu=0, \quad\left(U_{|x||x|}-2|x| U_{|x|}, \operatorname{Im} U_{|x||x|}\right) \neq(0,0),
$$

is analogous to that from Remark 45 for Case 3 of Theorem 44, and similarly excludes the values of $V$ being $G^{\sim}$-equivalent to those from Cases 6 and 16. Nevertheless, the maximality condition for Case 6 is modified, in comparison with the condition from Remark 45 for Case 4 of Theorem 44, to

$$
\mu \neq 0 \quad \text { or, if } \mu=0, \quad\left(U_{|x||x|}-2|x| U_{|x|}, \operatorname{Im} U_{|x||x|}\right) \neq(0,0) \quad \text { and } \quad\left(|x|^{2} U\right)_{|x|} \neq 0
$$

to single out the potentials from Case 4 that are $G^{\sim}$-inequivalent to those in both Cases 7 and 16. Inequalities to be satisfied by tuples of parameter functions appearing in listed potentials and depending only on the variable $t$ also become more complicated. Thus, the maximality condition in Case 19 is that $\left(\left(\alpha t^{2}+\beta t+\gamma\right) h^{00}\right)_{t} \neq 2 \alpha \lambda^{\prime}$ for any nonzero constant tuple $(\alpha, \beta, \gamma)$, 
which excludes further Lie symmetry extensions $G^{\sim}$-equivalent to Cases $20-22$. For Case 8 , the maximality condition is $\left(U_{222}, \operatorname{Im} U_{2}\right) \neq(0,0)$ and $\left(\left(\alpha t^{2}+\beta t+\gamma\right) U\right)_{t} \neq 2 \alpha \lambda^{\prime}$ for any nonzero constant tuple $(\alpha, \beta, \gamma)$. In Cases 9-11 the maximality condition is $\left(U_{\varpi \varpi \varpi}, \operatorname{Im} U_{\varpi}\right) \neq(0,0)$ and $\left(\varpi^{2} U\right)_{\varpi} \neq 0$, and this excludes values of $V$ that are $G^{\sim}$-equivalent to those from Cases 12 and 16-23. Similarly, potentials from Cases 13-15 are $G^{\sim}$-inequivalent to those from Cases 1623 if and only if $\left(U_{\omega_{2} \omega_{2} \omega_{2}}, \operatorname{Im} U_{\omega_{2}}\right) \neq(0,0)$. This condition is necessary and sufficient for the maximality of Lie symmetry extensions given in Cases 14 and 15, but for Case 13 we need a further condition to guarantee the inequivalence with potentials from Cases 14 and 15.

Remark 52. To make the presentation in Theorem 50 consistent with Theorems 44 and 47, we avoid scalings of constant parameters and alternating their signs as well as gauging some parameter functions. In contrast to the classes $\mathcal{V}^{f}$ and $\mathcal{P}_{0}^{\delta}$, the class $\mathcal{P}_{\lambda}^{\delta}$ admits scaling equivalence transformations and even more general transformations with nonconstant $T_{t}$. As a result, we can set $\mu \in\{0,1\}$ in Cases 5 and $6, \mu \geqslant 0$ in Case 15, $\kappa=1$ in Cases 2, 3, 14, 15, 17 and 18, $h^{11}=0\left(\right.$ or $\left.h^{22}=0\right)$ in Case $16, \operatorname{Im} U \geqslant 0$ if $\operatorname{Im} \delta=0$ in Cases 7 and $12, \nu= \pm 1$ if $\operatorname{Im} \delta \neq 0$ and $\nu=1$ if $\operatorname{Im} \delta=0$ in Case 20, $\nu \geqslant \lambda^{\prime}$ in Case 21 using the equivalence transformation $\mathcal{D}\left(-t^{-1}\right) \mathcal{I}\left(2 \lambda^{-1} \ln |t|\right)$, and $\nu \geqslant 0$ if $\operatorname{Im} \delta=0$ in Case 22 .

Proof. As in the proofs of Theorems 44 and 47, we follow the discussion from the corresponding Section 5.3 and single out different classification cases using the invariant integers $r_{1}, k_{2}$ and $k_{3}$. For the class $\mathcal{P}_{\lambda}^{\delta}$, the general form of basis vector fields of $\mathfrak{g}_{V}$ from the complement of $\mathfrak{g}^{\cap}$ is

$$
Q^{s}=D^{\lambda}\left(\tau^{s}\right)+\kappa_{s} J+P\left(\chi^{s 1}, \chi^{s 2}\right)+\sigma^{s} M,
$$

where the range of $s$ is equal to $\operatorname{dim} \mathfrak{g}_{V}-1, \kappa_{s}$ are real constants, $\tau^{s}, \chi^{s 1}, \chi^{s 2}$ and $\sigma^{s}$ are real-valued functions of $t$, and the parameter tuples $\left(\tau^{s}, \kappa_{s}, \chi^{s 1}, \chi^{s 2}\right)$ are linearly independent. Substituting $\left(\tau^{s}, \kappa_{s}, \chi^{s 1}, \chi^{s 2}, \sigma^{s}\right)$ into the classifying condition (25), we derive the system of $\operatorname{dim} \mathfrak{g}_{V}-1$ equations with respect to $V$,

$$
\begin{aligned}
\tau^{s} V_{t}+ & \frac{1}{2} \tau_{t}^{s} x_{a} V_{a}+\kappa_{s}\left(x_{1} V_{2}-x_{2} V_{1}\right)+\chi^{s a} V_{a}+\tau_{t}^{s} V \\
= & \frac{1}{8} \tau_{t t t}^{s} x_{a} x_{a}+\frac{1}{2} \chi_{t t}^{s a} x_{a}+\sigma_{t}^{s}+i \lambda^{\prime} \tau_{t t}^{s} .
\end{aligned}
$$

$\boldsymbol{r}_{\mathbf{1}}=\boldsymbol{k}_{\mathbf{2}}=\mathbf{0}$. The classification of Lie-symmetry extensions depends only on $k_{3}$, and Lemma 42 reduces it to the classification of finite-dimensional algebras of vector fields on the real line. The algebra $\mathfrak{g}_{V}$ contains $k_{3}$ vector fields $Q^{s}, s=1, \ldots, k_{3}$, where $\tau^{s}$ are linearly independent.

The condition $k_{3}=0$ means that there is no Lie-symmetry extension, $\mathfrak{g}_{V}=\mathfrak{g}^{\cap}=\langle M\rangle$, and we have Case 0.

If $k_{3}=1$, then up to $\pi_{*} G^{\sim}$-equivalence we can set $\tau^{1}=1, \chi^{11}=\chi^{12}=\sigma^{1}=0$ and, if $\delta_{2}=0$, then $\kappa_{3} \geqslant 0$. Depending on whether or not $\kappa$ is zero, this splits into Cases 2 and 3 .

For $k_{3} \geqslant 2$, modulo $\pi_{*} G^{\sim}$-equivalence and changing basis of $\mathfrak{g}_{V}$ we can assume that $\left(\tau^{1}, \tau^{2}\right)=$ $(1, t)$ and further set $\chi^{1 a}=\sigma^{1}=0$. In view of the equations $\kappa_{1}=0, \chi_{t}^{2 a}=\sigma_{t}^{2}=0$ following from the condition $\left[Q^{1}, Q^{2}\right] \in \mathfrak{g}_{V}$, we can annihilate $\sigma^{2}$ and $\left(\chi^{21}, \chi^{22}\right)$ by combining $Q^{2}$ with $M$ and by acting with $\mathcal{P}_{*}\left(2 \chi^{21}, 2 \chi^{22}\right)$ on $\mathfrak{g}_{V}$, respectively. Solving the equations (39) with $s=1,2$, we obtain the expression for $V$ from Case 4. Then the classifying condition (25) with such $V$ implies that there is no further Lie-symmetry extension for $\lambda \neq 2$, and for $\lambda=2$ the algebra $\mathfrak{g}_{V}$ contains one more vector field, $Q^{3}=D^{\lambda}\left(t^{2}\right)$.

$\boldsymbol{r}_{\mathbf{1}}=\mathbf{0}, \boldsymbol{k}_{2}=\mathbf{1}$. The algebra $\mathfrak{g}_{V}$ necessarily contains the vector field $Q^{0}$ with $\tau^{1}=0$ and $\kappa_{1}=1$, where $\chi^{0 a}$ can be set to zero up to $\pi_{*} G^{\sim}$-equivalence. The further consideration depends on the value of $k_{3}$.

$k_{3}=0$. Modulo $\pi_{*} G^{\sim}$-equivalence, we can assume that $\sigma^{0}=\mu t$ with $\mu \in\{0,1\}$, which leads to Case 5 . 
$k_{3}=1$. The algebra $\mathfrak{g}_{V}$ additionally contains the vector field $Q^{1}$ with $\tau^{1} \neq 0$. Up to $\pi_{*} G^{\sim}$ equivalence we can set $\tau^{1}=1, \sigma^{1}=0$. The condition $\left[Q^{0}, Q^{1}\right] \in \mathfrak{g}_{V}$ implies $\chi^{1 a}=0, \sigma_{t}^{0}=\mu=$ const and thus, linearly combining $Q^{0}$ with $M$, we set $\sigma^{0}=\mu t$ and obtain Case 6 .

$k_{3} \geqslant 2$. Modulo $\pi_{*} G^{\sim}$-equivalence and changing basis of $\mathfrak{g}_{V}$, we can again assume that $\left(\tau^{1}, \tau^{2}\right)=$ $(1, t)$. We successively set $\kappa_{1}=\kappa_{2}=0$ by linearly combining $Q^{1}$ and $Q^{2}$ with $Q^{0}$ and $\sigma^{1}=0$ by acting with appropriate $\mathcal{M}_{*}(\Sigma)$ on $\mathfrak{g}_{V}$. The condition $\left[Q^{0}, Q^{s}\right] \in \mathfrak{g}_{V}, s=1,2$, gives $\chi^{s a}=0$, $\sigma_{t}^{0}=0$. Therefore, we can set $\sigma^{0}=0$ by linearly combining $Q^{0}$ with $M$. The simultaneous integration of the equations (39) with $s=0,1,2$ provides the expression for $V$ from Case 7 . In view of the classifying condition (25) with such $V$, there is no further Lie-symmetry extension for $\lambda \neq 2$, and the algebra $\mathfrak{g}_{V}$ additionally contains the vector field $Q^{3}=D^{\lambda}\left(t^{2}\right)$ if $\lambda=2$.

$\boldsymbol{r}_{\mathbf{1}}=1$. Then $k_{2}=0$, and the algebra $\mathfrak{g}_{V}$ contains, in addition to $M$, at least a vector field $Q^{1}=$ $P\left(\chi^{11}, \chi^{12}\right)+\sigma^{1} M$. We again have two possibilities for the tuple $\left(\chi^{11}, \chi^{12}\right)$.

1. $\chi_{t}^{11} \chi^{12}=\chi^{11} \chi_{t}^{12}$. That is, the tuple $\left(\chi^{11}, \chi^{12}\right)$ is proportional to a constant tuple. Up to $\pi_{*} G^{\sim}$-equivalence we can set $Q^{1}=P(1,0)$. Then the equation (39) with $s=1$ is $V_{1}=0$, in view of which the classifying condition (25) implies that the vector field $P(t, 0)$ also belongs to $\mathfrak{g}_{V}$. Thus we have $V=U\left(t, x_{2}\right)$, where $\left(U_{222}, \operatorname{Im} U_{2}\right) \neq(0,0)$, since otherwise $r_{1}=2$, cf. the case $r_{1}=2$ below. Consider the subclass $\mathcal{U}$ of the class $\mathcal{P}_{\lambda}^{\delta}$ of equations with potentials of this form, which is singled out from $\mathcal{P}_{\lambda}^{\delta}$ by the constraints $V_{1}=0$ and $\left(V_{222}, \operatorname{Im} V_{2}\right) \neq(0,0)$. This subclass is normalized and its equivalence group is singled out from the equivalence group $G^{\sim}$ of $\mathcal{P}_{\lambda}^{\delta}$ by the conditions that $T$ is fractional linear in $t, O \in\left\{\operatorname{diag}\left(\varepsilon_{1}, \varepsilon_{2}\right) \mid \varepsilon_{1}, \varepsilon_{2}= \pm 1\right\}$, and $\mathcal{X}^{1}$ is affine in $T$. We successively split the equations (39) with $V=U\left(t, x_{2}\right)$ with respect to $x_{1}$ and $U_{2}$, obtaining $\tau_{t t t}^{s}=0, \kappa_{s}=0$ and $\chi_{t t}^{s 1}=0$. Therefore, linearly combining $Q^{s}, s \neq 1,2$ with $Q^{1}$ and $Q^{2}$, we can annihilate $\chi^{s 1}$ for $s \neq 1,2$. The classification of Lie-symmetry extensions within the subclass $\mathcal{U}$ is reduced to the classification of subalgebras of the algebra $\left\langle\partial_{t}, t \partial_{t}, t^{2} \partial_{t}\right\rangle \simeq \operatorname{sl}(2, \mathbb{R})$. A list of inequivalent subalgebras of this algebra is exhausted by

$$
\{0\}, \quad\left\langle\partial_{t}\right\rangle, \quad\left\langle t \partial_{t}\right\rangle, \quad\left\langle\left(t^{2}+1\right) \partial_{t}\right\rangle, \quad\left\langle\partial_{t}, t \partial_{t}\right\rangle, \quad\left\langle\partial_{t}, t \partial_{t}, t^{2} \partial_{t}\right\rangle
$$

The zero subalgebra corresponds to Case 8 , where $k_{3}=0$, which is the general case for the subclass $\mathcal{U}$. For $k_{3}=1$, we should consider the listed one-dimensional subalgebras. In other words, here the additional Lie symmetry extension is provided by a vector field $Q^{3}$ with $\tau^{3} \in\left\{1, t, t^{2}+1\right\}$. Acting by $\mathcal{P}_{*}\left(0, \mathcal{X}^{2}\right)$ and $\mathcal{M}_{*}(\Sigma)$ with appropriate values of the parameter functions $\mathcal{X}^{2}$ and $\Sigma$, we can make $\chi^{32}$ and $\sigma^{3}$ vanishing, deriving Cases $9-11$. If $k_{3} \geqslant 2$, then modulo $\pi_{*} G^{\sim}$ equivalence the Lie-symmetry extension contains at least two vector fields $Q^{3}$ and $Q^{4}$ with $\tau^{3}=1$ and $\tau^{4}=t$. We again reduce $Q^{3}$ to the form $Q^{3}=D(1)$. The condition $\left[Q^{3}, Q^{4}\right] \in \mathfrak{g}_{V}$ implies that $\chi_{t}^{42}=\sigma_{t}^{4}=0$. This is why we can annihilate $\sigma^{4}$ and $\chi^{42}$ by combining $Q^{4}$ with $M$ and by acting with $\mathcal{P}_{*}\left(0,2 \chi^{42}\right)$ on $\mathfrak{g}_{V}$, respectively. Then the equations (39) with $s=3,4$ jointly integrate to $V=U x_{2}^{-2}$, and it is obvious that $k_{3}$ is equal to either 2 or 3 if $\lambda \neq 2$ or $\lambda=2$, respectively. This gives Case 12 .

2. $\chi_{t}^{11} \chi^{12} \neq \chi^{11} \chi_{t}^{12}$, i.e., the tuple $\left(\chi^{11}, \chi^{12}\right)$ is not proportional to a constant tuple.

If $k_{3}=0$, then we can, up to $\pi_{*} G^{\sim}$-equivalence, make $Q^{1}=P(h \cos t, h \sin t)$, where $h(t)$ is a nonvanishing smooth function of $t$, which gives Case 13 .

The condition $k_{3}=1$ means that the algebra $\mathfrak{g}_{V}$ contains exactly one (up to linear combining) vector field $Q^{2}$ with $\tau^{2} \neq 0$. Up to $\pi_{*} G^{\sim}$-equivalence, the parameter functions $\chi^{1 a}, \sigma^{1}$ can be set to zero, and $Q^{2}$ reduces to $Q^{2}=D(1)+\kappa_{2} J$. Further consideration, which is similar to that in the respective case of the proof of Theorem 44, leads to Cases 14 and 15.

The case $k_{2} \geqslant 2$ is not possible. Indeed, otherwise the algebra $\mathfrak{g}_{V}$ would contain, modulo $\pi_{*} G^{\sim}$-equivalence, vector fields $Q^{2}$ with $\tau^{2}=1$ and $Q^{3}$ with $\tau^{3}=t$. The condition $\left[Q^{2}, Q^{3}\right] \in \mathfrak{g}_{V}$ gives $\kappa_{2}=0$. Then the condition $\left[Q^{2}, Q^{1}\right] \in \mathfrak{g}_{V}$ implies that $\left(\chi_{t}^{11}, \chi_{t}^{12}\right) \in\left\langle\left(\chi^{11}, \chi^{12}\right)\right\rangle$, which contradicts case's assumption. 
$\boldsymbol{r}_{\mathbf{1}}=\mathbf{2}$. Following the corresponding case of the proof of Theorem 44, up to $G^{\sim}$-equivalence we reduce the potential $V$ to the form (29) and substitute it into the equations (39). Then we split the obtained equations with respect to different powers of $\left(x_{1}, x_{2}\right)$ and derive the systems

$$
\begin{aligned}
& \tau^{s} h_{t}^{11}+2 \tau_{t}^{s} h^{11}+2 \kappa_{s} h^{12}=\frac{\tau_{t t t}}{2}, \quad \tau^{s} h_{t}^{22}+2 \tau_{t}^{s} h^{22}-2 \kappa_{s} h^{12}=\frac{\tau_{t t t}}{2}, \\
& \tau^{s} h_{t}^{12}+2 \tau_{t}^{s} h^{12}+\kappa_{s}\left(h^{22}-h^{11}\right)=0, \quad \tau^{s} h_{t}^{00}+\tau_{t}^{s} h^{00}=\tau_{t t}, \\
& \chi_{t t}^{s a}=h^{a b} \chi^{s b}, \quad \sigma_{t}^{s}=0 .
\end{aligned}
$$

In view of the system (41), the algebra $\mathfrak{g}_{V}$ in fact contains, in addition to $M$, four vector fields $Q^{p}=P\left(\chi^{p 1}, \chi^{p 2}\right)$, where tuples $\left(\chi^{p 1}, \chi^{p 2}\right), p=1, \ldots, 4$, constitute a fundamental set of solutions of the system $\chi_{t t}^{a}=h^{a b} \chi^{b}$. Moreover, we can set $\chi^{s 1}=\chi^{s 2}=\sigma^{s}=0$ for $s \neq 1, \ldots, 4$ by linearly combining $Q^{s}$ with $Q^{1}, \ldots, Q^{4}$ and $M$. Note that due to (40), the condition $k_{2}=1$ is equivalent to having $h^{11}=h^{22}$ and $h^{12}=0$.

The consideration of the cases $\left(r_{1}, k_{2}, k_{3}\right)=(2,0,0)$ and $\left(r_{1}, k_{2}, k_{3}\right)=(2,0,1)$ is similar to that in the proof of Theorem 44 and leads to Cases 16, 17 and 18. The case $\left(r_{1}, k_{2}, k_{3}\right)=(2,0,2)$ is impossible. Indeed, otherwise the algebra $\mathfrak{g}_{V}$ could contain, modulo $\pi_{*} G^{\sim}$-equivalence, vector fields $Q^{5}$ and $Q^{6}$ with $\tau^{5}=1$ and $\tau^{6}=t$. The condition $\left[Q^{5}, Q^{6}\right] \in \mathfrak{g}_{V}$ implies that $\kappa_{1}=0$, and thus the system of equations (40) with $s=5,6$ admits, as a system with respect to $h$ 's, only the zero solution, which contradicts the condition $k_{2}=0$.

In view of Corollary 35, the condition $k_{2}=1$ implies that, modulo $G^{\sim}$-equivalence, the potential $V$ does not depend on $x$ and is purely imaginary, i.e., $V=i h^{00}(t)$, where $h^{00}$ is a smooth real-valued function of $t$. Substituting $V$ into the classifying condition (25) and splitting with respect to the powers of $x$, we obtain the equations $\tau_{t t t}=0, \chi_{t t}^{a}=0, \sigma_{t}=0$, $\left(\tau h^{00}\right)_{t}=\lambda^{\prime} \tau_{t t}$. They imply that the vector fields $J, P(1,0), P(t, 0), P(0,1), P(0, t)$ belong to the maximal Lie invariance algebra of any equation with potentials of the above form. Additional $G^{\sim}$-inequivalent Lie-symmetry extensions are related to inequivalent nonzero subalgebras of the algebra $\left\langle D(1), D^{\lambda}(t), D^{\lambda}\left(t^{2}\right)\right\rangle$, which are exhausted by $\langle D(1)\rangle,\left\langle D^{\lambda}(t)\right\rangle,\left\langle D^{\lambda}\left(t^{2}+1\right)\right\rangle$, $\left\langle D(1), D^{\lambda}(t)\right\rangle$ and the entire algebra itself. This leads to Cases 19-23.

Remark 53. Cases 10, 11, 21 and 22 of Theorem 50 can be replaced by the corresponding $G^{\sim}$-equivalent cases with $t$-independent potentials,

$$
\begin{aligned}
& 10^{\prime} . V=\frac{1}{4} x_{1}^{2}+\tilde{U}\left(x_{2}\right): \quad \mathfrak{g}_{V}=\left\langle M, P\left(e^{-t}, 0\right), P\left(e^{t}, 0\right), D(1)\right\rangle, \\
& 11^{\prime} . V=-\frac{1}{4} x_{1}^{2}+\tilde{U}\left(x_{2}\right): \mathfrak{g}_{V}=\langle M, P(\cos t, 0), P(\sin t, 0), D(1)\rangle, \\
& 21^{\prime} . V=\frac{1}{4}|x|^{2}+i \tilde{\nu}: \quad \mathfrak{g}_{V}=\left\langle M, P\left(e^{-t}, 0\right), P\left(e^{t}, 0\right), P\left(0, e^{-t}\right), P\left(0, e^{t}\right), J, D(1)\right\rangle, \\
& 22^{\prime} . V=-\frac{1}{4}|x|^{2}+i \nu: \quad \mathfrak{g}_{V}=\langle M, P(\cos t, 0), P(\sin t, 0), P(0, \cos t), P(0, \sin t), J, D(1)\rangle .
\end{aligned}
$$

Here $\tilde{\nu}:=2\left(\nu-\lambda^{\prime}\right) \neq-2 \lambda^{\prime}$ in Case $21^{\prime}$ and $\left(\lambda^{\prime}, \nu\right) \neq(0,0)$ in Case 22'. The mappings of Cases $10^{\prime}, 21^{\prime}$ to Cases 10, 21 and of Cases $11^{\prime}, 22^{\prime}$ to Cases 11, 22 are realized by the equivalence transformations $\mathcal{D}\left(e^{2 t}\right) \mathcal{I}\left(-2 \lambda^{-1} t\right)$ and $\mathcal{D}(\tan t) \mathcal{I}\left(\lambda^{-1} \ln \cos ^{2} t\right)$, respectively.

\subsection{Classification list for the entire class}

Summing up the results of this section, we obtain the following assertion.

Theorem 54. A complete list of $\mathcal{G}_{\tilde{v}}$-inequivalent Lie symmetry extensions in the class $\mathcal{V}$ with $n=2$ is the union of the group classification lists for the subclasses $\mathcal{V}^{\prime}, \mathcal{P}_{0}$ and $\mathcal{P}_{\lambda}, \lambda \in \mathbb{R}_{\neq 0}$, up to $G_{\tilde{V}^{\prime}}{ }^{-}, G_{\mathcal{P}_{0}}-$ and $G_{\mathcal{P}_{\lambda}}$-equivalences, which are presented in Theorems 44,47 and 50, respectively.

It is easy to select $\mathcal{G} \tilde{v}$-inequivalent Lie symmetry extensions with stationary potentials. These are cases involving the vector field $D(1)$, which are exhausted by Cases 1, 4, 6, 11 and 14 of Theorem 44, Cases 1, 4, 6, 11 and 14 of Theorem 47, Cases 1, 3, 4, 6, 7, 9, 12, 17, 20 and 23 of Theorem 50 and the modified Cases $10^{\prime}, 11^{\prime}, 21^{\prime}$ and $22^{\prime}$ given in Remark 53. 


\section{Acknowledgements}

The authors are pleased to thank Peter Basarab-Horwath, Vyacheslav Boyko, Michael Kunzinger, Anatoly Nikitin, Galyna Popovych, Dmytro Popovych, Alexander Sakhnovich and Olena Vaneeva for stimulating discussions. The research of C.K. was supported by International Science Programme (ISP) in collaboration with Eastern Africa Universities Mathematics Programme (EAUMP). The research of R.O.P. was supported by the Austrian Science Fund (FWF), projects P25064, P29177 and P30233. The authors are grateful to the Abdus Salam International Centre for Theoretical Physics (ICTP) for hospitality and excellent scientific environment.

\section{References}

[1] Anco S.C. and Feng W., Group-invariant solutions of semilinear Schrödinger equations in multi-dimensions, J. Math. Phys. 54 (2013), 121504, arXiv:1301.5529.

[2] Anco S.C., Feng W. and Wolf T., Exact solutions of semilinear radial Schrödinger equations by separation of group foliation variables, J. Math. Anal. Appl. 427 (2015), 759-786, arXiv:1408.3751.

[3] Baumann G. and Nonnenmacher T.F., Lie transformations, similarity reduction, and solutions for the nonlinear Madelung fluid equations with external potential, J. Math. Phys. 28 (1987), 1250-1260.

[4] Belmonte-Beitia J., Pérez-García V.M., Vekslerchik V. and Torres P.J., Lie symmetries, qualitative analysis and exact solutions of nonlinear Schrödinger equations with inhomogeneous nonlinearities, Discrete Contin. Dyn. Syst. Ser. B 9 (2008), 221-233, arXiv:0801.1437.

[5] Białynicki-Birula I. and Sowiński T., Solutions of the logarithmic Schrödinger equation in a rotating harmonic trap. Nonlinear waves: classical and quantum aspects. NATO Sci. Ser. II Math. Phys. Chem. 153, Kluwer Academic, Dordrecht, 2004, 99-106, arXiv:quant-ph/0310195.

[6] Bihlo A., Dos Santos Cardoso-Bihlo E. and Popovych R.O., Complete group classification of a class of nonlinear wave equations, J. Math. Phys. 53 (2012), 123515, arXiv:1106.4801.

[7] Bluman G., Simplifying the form of Lie groups admitted by a given differential equation, J. Math. Anal. Appl. 145 (1990), 52-62.

[8] Bluman G.W., Cheviakov A.F. and Anco S.C., Application of symmetry methods to partial differential equations, Springer, New York, 2010.

[9] Boyer C.P., The maximal 'kinematical' invariance group for an arbitrary potential, Helv. Phys. Acta 47 (1974), 589-605.

[10] Boyer C.P., Sharp R.T. and Winternitz P., Symmetry breaking interactions for the time dependent Schrödinger equation, J. Math. Phys. 17 (1976), 1439-1451.

[11] Clarkson P.A., Dimensional reductions and exact solutions of a generalized nonlinear Schrödinger equation, Nonlinearity 5 (1992), 453-472.

[12] Clarkson P.A. and Hood S., Symmetry reductions of a generalized, cylindrical nonlinear Schrödinger equation, J. Phys. A 26 (1993), 133-150.

[13] Doebner H.D., Goldin G.A. and Nattermann P., Gauge transformations in quantum mechanics and the unification of nonlinear Schrödinger equations, J. Math. Phys. 40 (1999), 49-63.

[14] Florjańczyk M. and Gagnon L., Exact solutions for a higher-order nonlinear Schrödinger equation, Phys. Rev. A 41 (1990), 4478-4485.

[15] Florjańczyk M. and Gagnon L., Dispersive-type solutions for the Eckhaus equation, Phys. Rev. A 45 (1992), $6881-6883$.

[16] Fröhlich J., Gustafson S., Jonsson B.L.G. and Sigal I.M., Solitary wave dynamics in an external potential, Comm. Math. Phys. 250 (2004), 613-642, arXiv:math-ph/0309053.

[17] Fushchych W.I. and Cherniha R.M., Galilei-invariant nonlinear systems of evolution equations, J. Phys. A 28 (1995), 5569-5579.

[18] Fushchych W.I. and Chopyk V.I., Symmetry and non-Lie reduction of the nonlinear Schrödinger equation, Ukr. Mat. Zh. 45 (1993), 539-551 (Ukrainian); translation in Ukr. Math. J. 45 (1993), 581-597.

[19] Fushchych W., Chopyk V., Nattermann P. and Scherer W., Symmetries and reductions of nonlinear Schrödinger equations of Doebner-Goldin type, Rep. Math. Phys. 35 (1995), 129-138.

[20] Fushchych W.I. and Moskaliuk S.S., On some exact solutions of the nonlinear Schrödinger equations in three spatial dimensions, Lett. Nuovo Cim. 31 (1981), 571-576. 
[21] Fushchych W.I., Serov N.I., On some exact solutions of three-dimensional non-linear Schrödinger equation, J. Phys. A 20 (1987), L929-L933.

[22] Gagnon L., Self-similar solutions for a coupled system of nonlinear Schrödinger equations, J. Phys. A 25 (1992), 2649-2667.

[23] Gagnon L. and Winternitz P., Lie symmetries of a generalised non-linear Schrödinger equation: I. The symmetry group and its subgroups, J. Phys. A 21 (1988), 1493-1511.

[24] Gagnon L. and Winternitz P., Lie symmetries of a generalised nonlinear Schrödinger equation: II. Exact solutions, J. Phys. A 22 (1989), 469-497.

[25] Gagnon L., Grammaticos B., Ramani A. and Winternitz P., Lie symmetries of a generalised nonlinear Schrödinger equation: III. Reductions to third-order ordinary differential equations, J. Phys. A 22 (1989), 499-509.

[26] Gagnon L. and Winternitz P., Exact solutions of the cubic and quintic nonlinear Schrödinger equation for a cylindrical geometry, Phys. Rev. A (3) 39 (1989), 296-306.

[27] Gagnon L. and Winternitz P., Exact solutions of the spherical quintic nonlinear Schrödinger equation, Phys. Lett. A 134 (1989), 276-281.

[28] Gagnon L. and Winternitz P., Non-Painlevé reductions of nonlinear Schrödinger equations, Phys. Rev. A 42 (1990), 5029-5030.

[29] Gagnon L. and Wintenitz P., Symmetry classes of variable coefficient nonlinear Schrödinger equations, J. Phys. A 26 (1993), 7061-7076.

[30] García-Ripoll J.J., Pérez-García V.M. and Vekslerchik V., Construction of exact solutions by spatial translations in inhomogeneous nonlinear Schrödinger equations, Phys. Rev. E 64 (2001), 056602, arXiv:cond-mat/0106487.

[31] Güngör, F. Nonlinear evolution equations invariant under the Schrödinger group in three-dimensional spacetime, J. Phys. A 32 (1999), 977-988.

[32] Ibragimov N.H., Aksenov A.V., Baikov V.A., Chugunov V.A., Gazizov R.K. and Meshkov A.G., CRC handbook of Lie group analysis of differential equations. Vol. 2. Applications in engineering and physical sciences, edited by Ibragimov, CRC Press, Boca Raton, FL, 1995.

[33] Ivanova N., Symmetry of nonlinear Schrödinger equations with harmonic oscillator type potential, Proceedings of Insitute of Mathematics of NAS of Ukraine 43 (2002), Part 1, 149-150.

[34] Ivanova N.M., Popovych R.O. and Eshraghi H., On symmetry properties of nonlinear Schrödinger equations, Sveske Fiz. Nauka 18(A1) (2005), 451-456.

[35] Kengne E. and Vaillancourt R., Exact solutions of the Gross-Pitaevskii equation in periodic potential in the presence of external source, J. Math. Phys. 48 (2007), 073520.

[36] Kingston J.G. and Sophocleous C., On form-preserving point transformations of partial differential equations, J. Phys. A 31 (1998), 1597-1619.

[37] Kurujyibwami C., Basarab-Horwath P. and Popovych R.O., Algebraic method for group classification of (1+1)-dimensional linear Schrödinger equations, Acta Appl. Math. 157 (2018), 171-203, arXiv:1607.04118.

[38] Martina L., Soliani G. and Winternitz P., Partially invariant solutions of a class of nonlinear Schrödinger equations, J. Phys. A 25 (1992), 4425-4435.

[39] Miller W., Symmetry and separation of variables, Addison-Wesley, Reading, 1977.

[40] Niederer U., The maximal kinematical invariance group of the free Schrödinger equation, Helv. Phys. Acta. 45 (1972), 802-810.

[41] Niederer U., The maximal kinematical invariance group of the harmonic oscillator, Helv. Phys. Acta. 46 (1973), 191-200.

[42] Niederer U., The maximal kinematical invariance groups of Schrödinger equations with arbitrary potentials, Helv. Phys. Acta. 47 (1974), 167-172.

[43] Nikitin A.G., Kinematical invariance groups of the 3d Schrödinger equations with position dependent masses, J. Math. Phys. 58 (2017), 083508, 16 pp.

[44] Nikitin A.G. and Popovych R.O., Group classification of nonlinear Schrödinger equations, Ukr. Mat. Zh. 53 (2001), 1053-1060 (in Ukrainian); translation in Ukr. Math. J. 53 (2001), 1255-1265, arXiv:math-ph/0301009.

[45] Olver P.J., Applications of Lie groups to differential equations, Springer-Verlag, New York, 1986.

[46] Opanasenko S., Bihlo A. and Popovych R.O., Group analysis of general Burgers-Korteweg-de Vries equations, J. Math. Phys. 58 (2017), 081511, arXiv:1703.06932. 
[47] Ovsiannikov L.V., Group analysis of differential equations, Academic Press, New York, 1982.

[48] Özemir C. and Güngör F., Symmetry classification of variable coefficient cubic-quintic nonlinear Schrödinger equations, J. Math. Phys. 54 (2013), 023502.

[49] Pérez-García V.M., Torres P.J. and Konotop V.V., Similarity transformations for nonlinear Schrödinger equations with time-dependent coefficients, Phys. D 221 (2006), 31-36, arXiv:nlin.PS/0512061.

[50] Polyanin A.D. and Zaitsev V.F., Handbook of nonlinear partial differential equations, second edition, Chapman \& Hall/CRC, Boca Raton, FL, 2012.

[51] Popovych R.O., Classification of admissible transformations of differential equations, Collection of Works of Institute of Mathematics of NAS of Ukraine, 3 (2006), no. 2, 239-254.

[52] Popovych R.O., Normalized classes of nonlinear Schrödinger equations, Bulg. J. Phys. 33(s2) (2006), 211222.

[53] Popovych R.O. and Eshraghi H., Admissible point transformations of nonlinear Schrödinger equations, Proceedings of 10th International Conference in MOdern GRoup ANalysis (Larnaca, Cyprus, 2004), 167174.

[54] Popovych R.O., Ivanova N.M. and Eshraghi H., Lie symmetries of (1+1)-dimensional cubic Schrödinger equation with potential, Proceedings of Institute of Mathematics of NAS of Ukraine 50 (2004), Part 1, 219-224, arXiv:math-ph/0310039.

[55] Popovych R.O., Ivanova N.M. and Eshraghi H., Group classification of (1+1)-dimensional Schrödinger equations with potentials and power nonlinearities, J. Math.Phys. 45 (2004), 3049-3057, arXiv:math-ph/0311039.

[56] Popovych R.O., Kunzinger M. and Eshragi H., Admissible transformations and normalized classes of nonlinear Schrödinger equations, Acta Appl. Math. 109 (2010), 315-359, arXiv:math-ph/0611061.

[57] Popovych R.O., Kunzinger M. and Ivanova N.M., Conservation laws and potential symmetries of linear parabolic equations, Acta Appl. Math. 100 (2008), 113-185, arXiv:0706.0443.

[58] Rideau G. and Winternitz P. Evolution equations invariant under two-dimensional space-time Schrödinger group, J. Math. Phys. 34 (1993), 558-570.

[59] Sakhnovich A., Generalized Bäcklund-Darboux transformation: spectral properties and nonlinear equations, J. Math. Anal. Appl. 262 (2001), 274-306.

[60] Sakhnovich A., Non-self-adjoint Dirac-type systems and related nonlinear equations: wave functions, solutions, and explicit formulas, Integral Equations Operator Theory 55 (2006), 127-143.

[61] Sakhnovich A.L., On a new integrable nonlinear Schrödinger equation with a simple external potential and its explicit solutions, 2006, arXiv:nlin.SI/0610046.

[62] Sakhnovich A., Nonisospectral integrable nonlinear equations with external potentials and their GBDT solutions, J. Phys. A 41 (2008), 155204, 15 pp., arXiv:0710.2260.

[63] Stoimenov S. and Henkel M., Dynamical symmetries of semi-linear Schrödinger and diffusion equations, Nuclear Phys. B $\mathbf{7 2 3}$ (2005), 205-233, arXiv:math-ph/0504028.

[64] Talanov V., Focusing of light in cubic media, JETP Lett. 11 (1970) 199-201.

[65] Vaneeva O.O., Bihlo A. and Popovych R.O., Equivalence groupoid and group classification of a class of nonlinear wave and elliptic equations, arXiv:2002.08939.

[66] Wittkopf A.D., Algorithms and Implementations for Differential Elimination, PhD thesis, Simon Fraser University, 2004.

[67] Wittkopf A.D. and Reid G.J., Fast differential elimination algorithms, Technical Report TR-00-06, Ontario Research Centre for Computer Algebra, 2000.

[68] Wittkopf A.D. and Reid G.J., Fast differential elimination in C: The CDiffElim environment, Comp. Phys. Comm. 139 (2001), 192-217.

[69] Zhdanov R. and Roman O., On preliminary symmetry classification of nonlinear Schrödinger equation with some applications of Doebner-Goldin models, Rep. Math. Phys. 45 (2000), 273-291. 